Алгебра и анализ

Tom 21 (2009), № 6
St. Petersburg Math. J.

Vol. 21 (2010), No. 6, Pages 903-956

S 1061-0022(2010)01125-7

Article electronically published on September 23, 2010

\title{
ERROR BOUNDS, DUALITY, AND THE STOKES PHENOMENON. I
}

\author{
V. P. GURARII
}

\begin{abstract}
We consider classes of functions uniquely determined by coefficients of their divergent expansions. Approximating a function in such a class by partial sums of its expansion, we study how the accuracy changes when we move within a given region of the complex plane. Analysis of these changes allows us to propose a theory of divergent expansions, which includes a duality theorem and the Stokes phenomenon as essential parts. In its turn, this enables us to formulate necessary and sufficient conditions for a particular divergent expansion to encounter the Stokes phenomenon. We derive explicit expressions for the exponentially small terms that appear upon crossing Stokes lines and lead to an improvement in the accuracy of the expansion.
\end{abstract}

\section{§1. INTRODUCTION}

The Stokes phenomenon plays an exceptional role in complex analysis, in particular, in asymptotic analysis. It is very important for applications in theoretical and mathematical physics.

This phenomenon was a stumbling block for Poincaré's asymptotic theory, which failed to explain it and which could not be used to evaluate the exponentially small Stokes' discontinuities.

The stream of papers developing various ideas in the explanation of the phenomenon is vast and continues to grow. See, for example, [1, 3, 4, 5, 6, 7, 8, and the list of publications can be continued. The title of the paper "Stokes phenomenon demystified", see [9], is indicative of its extraordinary nature. It is difficult to think of another discovery of the middle part of the 19th century which still needs to be demystified 150 years after it was reported for the first time.

In evaluating the position of fringes of supernumerary rainbows, Stokes 10 came upon the problem of approximation of an entire function (the Airy function) in the complex $z$-plane by multivalued functions (generated by formal solutions of Airy's differential equation). He discovered the existence of certain rays, Stokes rays, such that upon crossing these rays, one needs to add a term, exponentially small in $z$ as $z \rightarrow \infty$, to the approximation in order to retain the previous accuracy.

Nearly one hundred years later, Pokrovskiu and Khalatnikov published the paper 11 in which they computed an exponentially small reflection which is not detected by the WKB method, and which would come to be called "asymptotics beyond-all-orders"; see [5], especially the papers by M. Berry and M. Kruskal et al., and [12].

2010 Mathematics Subject Classification. Primary 30E15.

Key words and phrases. Asymptotic approximation, error bounds, Stokes phenomenon.

EDitorial NotE: The following text incorporates changes and corrections submitted by the author after the paper had already been published (in English) in the Russian original of this journal. 
Although the breakthrough in asymptotic analysis over the last two decades and the creation of hyperasymptotic analysis shed light on this topic, it is still desirable to develop a theory of divergent expansions which is as simple, universal, and intelligible as Poincaré's asymptotic theory, and which includes Stokes' phenomenon and asymptotics beyond all orders.

The author became aware of the possibility of this approach in working on the survey [13]: since we are dealing with approximation of analytic functions by partial sums of their divergent expansions, the Stokes phenomenon should be considered in a more general context as a part of approximation theory rather than asymptotic analysis. Our aim in the current paper is to initiate the development of such a theory, based upon the approach of Watson [14, Nevanlinna [15], and Carleman [16], and summarized by Hardy [17.

For convenience, our main results will be stated in $\S 2$ in their simplest form and then developed in detail in the subsequent sections. In $\S 3$ we state a version of the duality theorem. In $\S 4$ we study parametric integral representations in the theory of LaplaceMellin transforms. In $\S 5$ we derive integral representations for the remainders of the Laplace-Mellin transforms in terms of the basic kernel, called the fundamental basic terminant by Dingle [18, 19, 20]. In $\S 6$ we study further properties of the basic kernel, which we use systematically to prove our main results. In $\S 7$ we provide rigorous error bounds for the Laplace-Mellin transforms on both sides of the Stokes lines, and on the lines themselves. In $\S 8$ we derive explicit formulas for the exponentially small terms and asymptotics beyond all orders that arise upon crossing the Stokes lines and that lead to improvements in the estimates of $\S 7$. This allows us to prove the duality theorem. We also provide necessary and sufficient conditions for a given expansion to encounter the Stokes phenomenon. $\S 9$ contains historical notes on the Stirling expansion.

First, however, in $\S 1$ we describe the result of Nevanlinna [15] and provide the Stirling expansion for $\log \Gamma(z)$ on the imaginary line and upon crossing it with accurate error bounds; see $\S 9$.

1.1. Nevanlinna's theorem. In 1916, Nevanlinna proved the following theorem 2 see [15, 22, 23.

Theorem. Given $R \geq 0$ and $A>0$, let $P(z)$ be an analytic and single-valued function on the half-plane

$$
\mathcal{P}(R)=\{z \in \mathbb{C}: \operatorname{Re}\{z\}>R\} .
$$

Let $\left\{p_{0}, p_{1}, \ldots\right\}$ be a sequence of complex numbers such that, for $z \in \mathcal{P}(R)$,

$$
\left|P(z)-\sum_{k=0}^{n-1} \frac{p_{k}}{z^{k+1}}\right| \leq \frac{M n !}{A^{n}|z|^{n+1}}, \quad n=0,1, \ldots,
$$

where $M>0$ is independent of $n$ and $z$, but may depend on $P(z)$.

It follows that there exists a function $F(t)$ analytic in the region

$$
\mathcal{D}(A)=\{t \in \mathbb{C}: \operatorname{dist}\{t,(0,+\infty)\} \leq A\},
$$

shown in Figure 1, and such that, inside every subregion $\mathcal{D}\left(A^{\prime}\right) \subset \mathcal{D}(A), 0<A^{\prime}<A$, we have

$$
|F(t)|<K\left(A^{\prime}\right) e^{R|t|}
$$

\footnotetext{
${ }^{1}$ Curiously, citing [21], Hardy [17] did not mention this result of Nevanlinna.

${ }^{2}$ In fact, F. Nevanlinna proved only the first part of the theorem, but in a more general form when $(n+1)$ ! in the right-hand side of (2) is replaced by $\Gamma\left(\frac{n+1}{k}+1\right), 0<k<\infty$.
} 


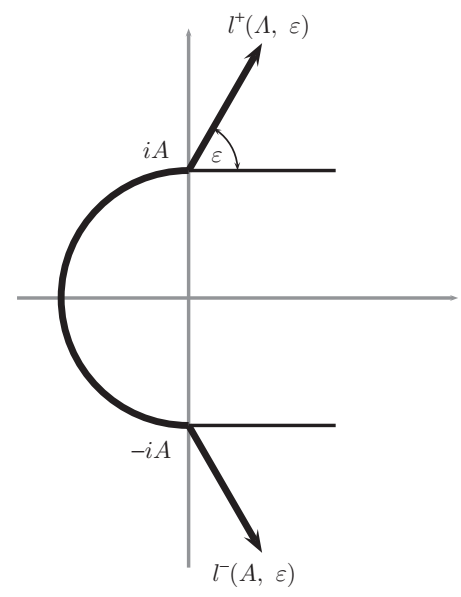

Figure 1. Regions $\mathcal{D}(A, \varepsilon)$ and $\mathcal{D}(A)=\mathcal{D}(A, 0)$ in the $t$-plane.

and

$$
P(z)=\int_{0}^{\infty} e^{-t z} F(t) d t, \quad p_{n}=F^{(n)}(0), \quad n=0,1, \ldots
$$

Conversely, if $F(t)$ is analytic in $\mathcal{D}(A)$ and in every subregion $\mathcal{D}\left(A^{\prime}\right) \subset \mathcal{D}(A)$ satisfies estimate (4), then the function $P(z)$ given by (5) is analytic in the half-plane $\mathcal{P}(R)$, and, setting $p_{n}=F^{(n)}(0)$, for every $0<A^{\prime}<A$ we have the following system of estimates:

$$
\left|P(z)-\sum_{k=0}^{n-1} \frac{p_{k}}{z^{k+1}}\right| \leq \frac{M\left(A^{\prime}\right) n !}{\left(A^{\prime}\right)^{n}|z|^{n+1}}, \quad n=0,1, \ldots .
$$

Nevanlinna's theorem was strengthened in 24, where it was shown that for the validity of the first part of Nevanlinna's theorem, it suffices to assume that $P(z)$ is analytic and satisfies conditions (2) not in the entire half-plane but only in a certain subregion. This discovery may shed light on the fact that there is a certain mismatch between the two parts of Nevanlinna's theorem. While it is possible to find how singularities of $F(t)$ affect error bounds, for example, by improving the corresponding result by Jeffreys [25, it is totally unclear how to describe the singularities of $F(t)$ by using error bounds for $P(z)$.

Our aim is to extend Nevanlinna's theorem to the region $\mathcal{P}(R, \varepsilon), \varepsilon>0$, given by

$$
\mathcal{P}(R, \varepsilon)=\bigcup_{-\varepsilon<\theta<\varepsilon} e^{i \theta} \mathcal{P}(R)
$$

see Figure 2.

This generalization is especially important, because it is in studying the case where $\varepsilon>0$ that we encounter the Stokes phenomenon.

Nevanlinna's theorem leads to an immediate improvement of Watson's theorem on Borel summability; see [14, 23].

Assume that, given $a, 0<a<\infty$, the estimates

$$
\left|P(z)-\sum_{k=0}^{n-1} \frac{p_{k}}{z^{k+1}}\right| \leq \frac{M n !}{a^{n}|z|^{n+1}}, \quad n=0,1, \ldots,
$$




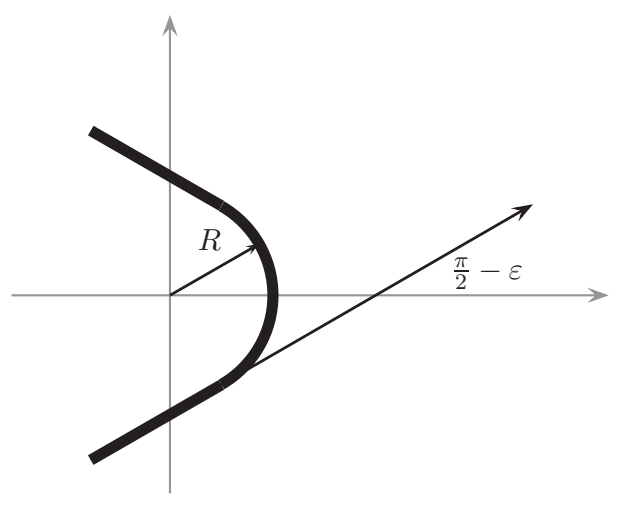

Figure 2. Region $\mathcal{P}(R, \varepsilon)$ in the $z$-plane.

are valid for $z \in \mathcal{P}(R, \varepsilon)$. Applying Nevanlinna's theorem for every half-plane $e^{i \theta} \mathcal{P}(R)$, we see that $F(t)$ given by (5) is analytic in the region

$$
D(A, \varepsilon)=\bigcup_{-\varepsilon<\theta<\varepsilon} e^{i \theta} \mathcal{D}(A) .
$$

Watson's theory of asymptotic expansions encountered serious objections from the experts in the field. These objections were summarized in the monographs [20] and [26]. We quote Dingle, page 408: “...the author searched the early literature deriving from Borel's work so as to check whether these interpretations were altogether new, and discovered a "near-miss" by Watson [14. He had applied Borel's original form to the terms - including early terms - of an asymptotic series away from any of its Stokes rays. His approach looks promising only for a relatively small class of alternating series deriving from exponential integrals, and it does not seem to have attracted more than theoretical interest - unlike Airey's later method, which was extensively called upon in early table-making and is still used". It should be noted that the improvement given by (8) does not eliminate Dingle's objection.

In [27, we presented the widest class of analytic functions whose elements are uniquely determined by the coefficients of their expansions. We discuss the largest conditions which might ensure extensions of both parts of Nevanlinna's theorems. Investigation of these conditions led the present author to discoveries described in the current paper.

1.2. Nonuniformity in $\arg z$ of error bounds of divergent expansions. From (2) it follows that

$$
\left|p_{n}\right| \leq \frac{M n !}{A^{n}}
$$

therefore, in general, the series $\sum_{k=0}^{\infty} p_{k} / z^{k+1}$ may be factorially divergent.

Numerous examples of classical divergent expansions show that, as opposed to convergent expansions, error bounds for divergent expansions are not uniform in $\arg z$.

We illustrate how error bounds change as $|\arg z|$ increases, using the Stirling expansion

$$
\begin{aligned}
& P(z)=\log \Gamma(z)-\left(z-\frac{1}{2}\right) \log z+z-\frac{1}{2} \log 2 \pi, \\
& P(z) \sim \sum_{k=1}^{\infty} \frac{B_{2 k}}{2 k(2 k-1)} \frac{1}{z^{2 k-1}}, \quad-\pi<\arg z<\pi, \quad z \rightarrow \infty,
\end{aligned}
$$


as a typical example. Here the $B_{2 k}$ are the Bernoulli numbers,

$$
B_{2 k}=\frac{(-1)^{k-1} 2(2 k) !}{(2 \pi)^{2 k}} \zeta(2 k)
$$

where $\zeta$ is the Riemann zeta-function.

Setting

$$
\mathrm{P}_{n}(z)=P(z)-\sum_{k=1}^{n-1} \frac{B_{2 k}}{2 k(2 k-1)} \frac{1}{z^{2 k-1}},
$$

and representing the remainder in the form

$$
\mathrm{P}_{n}(z)=T(z, n) \frac{B_{2 n}}{2 n(2 n-1) z^{2 n-1}},
$$

we have the following estimates:

- $-\frac{\pi}{4} \leq \arg z \leq \frac{\pi}{4}$

$$
|T(z, n)| \leq 1
$$

- $-\frac{\pi}{2}+\delta \leq \arg z \leq \frac{\pi}{2}-\delta, 0<\delta \leq \frac{\pi}{4}$,

$$
|T(z, n)| \leq \frac{1}{\sin 2 \delta},
$$

- $-\frac{\pi}{2} \leq \arg z \leq \frac{\pi}{2}, n \in \mathbb{N}$,

$$
|T(z, n)| \leq 1+\frac{1}{2} \sqrt{\pi\left(n+\frac{1}{2}\right)},
$$

- $-\frac{\pi}{2}-\varepsilon \leq \arg z \leq \frac{\pi}{2}+\varepsilon, 0 \leq \varepsilon<\frac{\pi}{2}, n \in \mathbb{N}$,

$$
|T(z, n)| \leq 1+\frac{1}{2} \sqrt{\pi\left(n+\frac{1}{2}\right)}+\frac{\sqrt{\pi(n+3)}}{(\cos \varepsilon)^{2 n-1}},
$$

and the number $\pi$ in the last pair of relations cannot be replaced by a smaller number.

We see that, as opposed to a convergent expansion, the error bounds for the Stirling expansion are not uniform in $\arg z$. The most noticeable changes happen upon crossing the rays $\arg z= \pm \pi$ in the counterclockwise and clockwise directions, respectively. The estimates clearly deteriorate. This example demonstrates the following general problem.

Given $R \geq 0,0 \leq a \leq b<\infty$, and $0 \leq \theta_{1}<\theta_{2}<\infty$, let $W\left(R, b, a, \theta_{1}, \theta_{2}\right)$ be the set of all functions $P(z)$ analytic in $\mathcal{P}\left(R, \theta_{2}\right)$ and with the corresponding sequences $\left\{p_{0}, p_{1}, \ldots\right\}$ satisfying the following conditions.

For every $P(z) \in W\left(R, b, a, \theta_{1}, \theta_{2}\right)$, there exist $a_{P}$ and $b_{P}$ satisfying the inequality $0<a<a_{P} \leq b_{P}<b<\infty$ and such that

- for $z \in \mathcal{P}\left(R, \theta_{1}\right)$ we have

$$
\left|P(z)-\sum_{k=0}^{n-1} \frac{p_{k}}{z^{k+1}}\right| \leq \frac{M_{1} n !}{\left(b_{P}\right)^{n}|z|^{n+1}}, \quad n=0,1, \ldots,
$$

- and for $z \in \mathcal{P}\left(R, \theta_{2}\right)$ we have

$$
\left|P(z)-\sum_{k=0}^{n-1} \frac{p_{k}}{z^{k+1}}\right| \leq \frac{M_{2} n !}{\left(a_{P}\right)^{n}|z|^{n+1}}, \quad n=0,1, \ldots .
$$

The positive constants $M_{1}$ and $M_{2}$ may depend on $P(z)$. 
It follows that $W\left(R, b, a, \theta_{1}, \theta_{2}\right)$ is a linear space that can be provided with a countable norm topology. The problem is that of developing techniques which would allow us to describe the corresponding dual spaces by using relation (5), to establish reasons for the deterioration of accuracy after crossing the rays $\arg z= \pm \theta_{1}$, and to extend both the first and the second parts of Nevanlinna's theorem to the elements of these spaces.

By using the Stokes phenomenon, it will be shown in $\S 8$ that only the cases of $\theta_{1}=\frac{n \pi}{2}$ and $\theta_{2}=\frac{n \pi}{2}+\varepsilon$, where $n \in \mathbb{Z}^{+}$and $\varepsilon$ is any number in $\left[0, \frac{\pi}{2}\right)$, are meaningful. We consider the case where $n=0$ and denote the corresponding linear space of functions satisfying (13) and (14) by $W(R, b, a, \varepsilon)$. The case where $\varepsilon=0$ corresponds to Nevanlinna's case, and $W(R, b, a, 0)=W(R, a)$. Other situations when $n \in \mathbb{N}$ can be treated similarly.

The expressions

$$
\mathrm{P}_{n}(z)=P(z)-\sum_{k=0}^{n-1} \frac{p_{k}}{z^{k+1}}, \quad n \in \mathbb{N}
$$

are called remainders or errors.

If $P(z) \in W(R, b, a, \varepsilon)$, then, for every $n \in \mathbb{N}$, from (13) and (14) it follows immediately that

$$
P(z) \sim \sum_{k=0}^{\infty} \frac{p_{k}}{z^{k+1}} \Longleftrightarrow \mathrm{P}_{n}(z)=O\left(\frac{1}{|z|^{n+1}}\right), \quad z \rightarrow \infty, \quad z \in \mathcal{P}(R, \varepsilon)
$$

and

$$
p_{n}=\lim z^{n+1} \mathrm{P}_{n}(z), \quad z \rightarrow \infty ;
$$

i.e., the coefficients $p_{n}$ are determined uniquely by $P(z)$. Estimates (13) show that $P(z)$ is determined uniquely by the sequence $\left\{p_{0}, p_{1}, \ldots\right\}$.

\section{§2. Statement of Results}

2.1. An extension of Nevanlinna's theorem. Given $A>0$, let

$$
\mathcal{D}(A)=\{t \in \mathbb{C}: \operatorname{dist}\{t,(0,+\infty)\} \leq A\} .
$$

We call this region determinative. The distance from a point on the boundary $\gamma(A)=$ $\partial \mathcal{D}(A)$ to the interval $[0, \infty)$ is equal to $A$. Given $0 \leq \varepsilon \leq \frac{\pi}{2}$, we introduce a family of regions $\mathcal{D}(A, \varepsilon)$ of the complex $t$-plane as a union of the form

$$
\mathcal{D}(A, \varepsilon)=\bigcup_{0 \leq|\theta|<\varepsilon} e^{i \theta} \mathcal{D}(A \cos \theta)
$$

so that $\mathcal{D}(A, \varepsilon) \rightarrow \mathcal{D}(A)$ as $\varepsilon \rightarrow 0$. It follows that the boundary $\gamma(A, \varepsilon)=\partial \mathcal{D}(A, \varepsilon)$ is represented as

$$
\gamma(A, \varepsilon)=l^{+}(A, \varepsilon) \cup C^{-}(A) \cup l^{-}(A, \varepsilon),
$$

where $l^{+}(A, \varepsilon)$ is the ray emanating from the point $i A$ and making an angle of $\varepsilon$ with the positive ray of the real line, $l^{-}(A, \varepsilon)$ is the reflection of $l^{+}(A, \varepsilon)$ in the real line, and $C_{A}^{-}$is the left half-circle of $C_{A}=\{t \in \mathbb{C}:|t|=A\}$; see Figure 1.

For given $A>0, R \geq 0$, and $0 \leq \varepsilon \leq \frac{\pi}{2}$, the space $B(R, A, \varepsilon)$ is defined as the set of all functions $F(t)$ that are analytic in the region $\mathcal{D}(A, \varepsilon)$ and satisfy the condition

$$
|F(t)| \leq K e^{R|t|}, \quad t \in \mathcal{D}(A, \varepsilon)
$$

where $K>0$ is independent of $t$ but may depend on $F(t)$. Then the following theorems are valid. 
Statement 1. Let the space $W(R, b, a, \varepsilon)$ be given as above, and let

$$
A=\sup b \text { for which (13) holds true for every } P(z) \in W(R, b, a, \varepsilon) \text {. }
$$

Then $a=A \cos \varepsilon$, and we denote

$$
W(R, A, \varepsilon)=W(R, A, A \cos \varepsilon, \varepsilon) .
$$

By using Statement 1 , for $n \in \mathbb{Z}^{+}$, conditions (13) and (14) can be rewritten in the form

$$
\left|P(z)-\sum_{k=0}^{n-1} \frac{p_{k}}{z^{k+1}}\right| \leq \frac{M_{1} n !}{A^{n}|z|^{n+1}}, \quad z \in \mathcal{P}(\varepsilon),
$$

and

$$
\left|P(z)-\sum_{k=0}^{n-1} \frac{p_{k}}{z^{k+1}}\right| \leq \frac{M_{2} n !}{(A \cos \varepsilon)^{n}|z|^{n+1}}, \quad z \in \mathcal{P}(R, \varepsilon) .
$$

The following theorem extends the first part of Nevanlinna's theorem.

Statement 2. Assume that $P(z) \in W(R, A, \varepsilon)$. Then there exists a function $F(t)$ analytic in the region $\mathcal{D}(A, \varepsilon)$ given by (18) and such that $F(t) \in B\left(R, A^{\prime}, \varepsilon\right)$ for every $0<A^{\prime}<A$, and

$$
P(z)=\int_{0}^{\infty} e^{-t z} F(t) d t
$$

The proof of these theorems is based on Nevanlinna's theorem and on the analysis presented in the next subsection.

Remark 1. In many classical situations we shall work with representations of the form

$$
P(z, \alpha)=z^{\alpha} \int_{0}^{\infty} e^{-t z} t^{\alpha} F(t) d t
$$

where $\operatorname{Re}\{\alpha\}>-1$ and $F(t) \in B\left(R, A^{\prime}, \varepsilon\right)$ for every $0<A^{\prime}<A$. It will be shown in the current paper that $F(t)$ generates a family $F(t, \alpha)$ such that $F(t, \alpha) \in B\left(R, A^{\prime}, \varepsilon\right)$ for every $0<A^{\prime}<A$ and

$$
P(z, \alpha)=\int_{0}^{\infty} e^{-t z} F(t, \alpha) d t .
$$

Thus, the definition of $P(z, \alpha)$ given by (25) reduces to the standard Laplace transformation.

One of our main goals is to study analytic continuation and the behavior of $P(z, \alpha)$ with respect to two complex variables, $z$ and $\alpha$. Keeping this goal in mind, first we provide this investigation for the function $F(t, \alpha)$ given by (26). This is done in $\S 4$.

2.2. Stokes' phenomenon. Let the rays $l^{-}(A, \varepsilon)$ and $l^{+}(A, \varepsilon)$ be defined by (19). Set

$$
E^{+}(z, A)=\int_{l^{-}(A, \varepsilon)} e^{-z t} F(t) d t
$$

and

$$
E^{-}(z, A)=\int_{l^{+}(A, \varepsilon)} e^{-z t} F(t) d t .
$$

The following theorems are valid. 
Statement 3. Assume that $P(z) \in W(R, A, \varepsilon), 0<\varepsilon \leq \frac{\pi}{2}$. Then for every $\theta, 0 \leq \theta \leq \varepsilon$, and for $z \in \mathcal{P}(R, \theta)$ we have

$$
\left|\mathrm{P}_{n}(z)\right| \leq \frac{M_{2} n !}{(A \cos \theta)^{n+1}|z|^{n+1}}, \quad n=0,1, \ldots .
$$

Comparison of (23) with (29) shows that estimates deteriorate upon crossing the rays $\arg z= \pm \frac{\pi}{2}$. The following theorem reveals the reasons for this deterioration.

Statement 4. Suppose $P(z) \in W(R, A, \varepsilon)$, and let $E^{+}(z, A)$ and $E^{-}(z, A)$ be given by (27) and (28), respectively. It follows that for every $A^{\prime}, 0<A^{\prime}<A$, and for $z \in$ $P(R, \varepsilon) \backslash \mathcal{P}(R)$ we have

$$
\left|E^{ \pm}\left(z, A^{\prime}\right)\right|=O\left(e^{-A^{\prime}|z|}\right), \quad z \rightarrow \infty .
$$

Moreover, for $\{z \in \mathcal{P}(R, \varepsilon) \backslash \mathcal{P}(R): \operatorname{Im}\{z\}>0\}$ we have

$$
\left|\mathrm{P}_{n}(z)-E^{+}\left(z, A^{\prime}\right)\right| \leq \frac{M_{1} n !}{\left(A^{\prime}\right)^{n+1}|z|^{n+1}}, \quad n=0,1, \ldots,
$$

and for $\{z \in \mathcal{P}(R, \varepsilon) \backslash \mathcal{P}(R): \operatorname{Im}\{z\}<0\}$ we have

$$
\left|\mathrm{P}_{n}(z)-E^{-}\left(z, A^{\prime}\right)\right| \leq \frac{M_{1} n !}{\left(A^{\prime}\right)^{n+1}|z|^{n+1}}, \quad n=0,1, \ldots
$$

This theorem shows that the rays $\arg z= \pm \frac{\pi}{2}$ should be regarded as Stokes rays, and the arising of exponentially small terms $E^{+}\left(z, A^{\prime}\right)$ and $E^{-}\left(z, A^{\prime}\right)$ upon crossing these lines should be regarded as occurrences of the Stokes phenomenon.

This theorem also shows that, in the left half-plane, the terms $E^{ \pm}\left(z, A^{\prime}\right)$ are the leading terms, and, in fact, these terms determine the behavior of the remainders.

Statement 5. Assume that $F(t) \in B(R, A, \varepsilon)$ and

$$
\int_{\gamma(A, \varepsilon)} e^{-R|\xi|}\left|\frac{F(\xi) d \xi}{\xi}\right|<\infty
$$

After setting

$$
P(z)=\int_{0}^{\infty} e^{-t z} F(t) d t
$$

it follows that $P(z) \in W(R, A, \varepsilon)$.

Subsequently, we conclude that if $F(t) \in B\left(R, A^{\prime}, \varepsilon\right)$ for every $0<A^{\prime}<A$, then $P(z) \in W\left(R, A^{\prime}, \varepsilon\right)$ for every $0<A^{\prime}<A$.

2.3. Necessary and sufficient conditions for Stokes' phenomenon. Let us call attention to the following pair of statements.

Statement 6. Assume that $0 \leq \varepsilon \leq \frac{\pi}{2}$. Then the series $\sum_{k=0}^{\infty} p_{k} / z^{k+1}$ for $P(z) \in$ $W(R, A, \varepsilon)$ is convergent if and only if $F(t)$, given by (5), is an entire function of exponential type.

Statement 7. Assume that $0<\varepsilon \leq \frac{\pi}{2}$, and let $P(z) \in W(R, A, \varepsilon)$. Then the estimates (2) are valid with arbitrary $0<A<\infty$ if and only if $F(t)$ is an entire function.

We note that the condition $\varepsilon>0$ in Statement 7 is essential, and that the statement: " $P(z) \in W(R, A, \varepsilon)$ for arbitrary $0<A<\infty \Longrightarrow F(t)$ is an entire function" follows from Nevanlinna's theorem. 


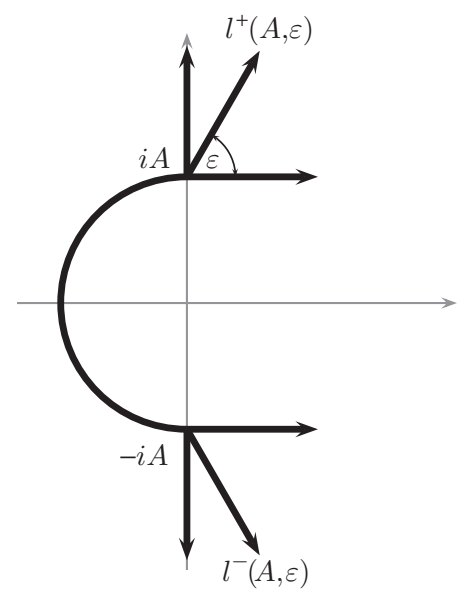

FIGURE 3. Expanding system of regions $\mathcal{D}(A, \varepsilon), 0 \leq \varepsilon \leq \frac{\pi}{2}$, in the $t$-plane.

Definition. We say that a function $P(z) \in W(R, A, \varepsilon)$ encounters Stokes' phenomenon in the region $\mathcal{P}(R, \varepsilon)$ given by (8) if the exponentially small terms $E^{ \pm}\left(z, A^{\prime}\right)$ given by (27), (28) appear in the region $\mathcal{P}(R, \varepsilon)$ and satisfy (31), (32), respectively, and

$$
\sup A^{\prime}<\infty \text {. }
$$

We introduce a system of regions in the $t$-plane, ordered by inclusion, $\subset$, which continues the system $\mathcal{D}(A, \varepsilon), 0 \leq \varepsilon \leq \frac{\pi}{2}$, given by (18). For $\frac{\pi}{2} \leq \varepsilon<\infty$, let

$$
\mathfrak{D}\left(A, \varepsilon+\frac{\pi}{2}\right)=\left\{t \in \mathbb{C}:-\varepsilon-\frac{\pi}{2}<\arg t<\varepsilon+\frac{\pi}{2},|t|>A\right\}
$$

and we have

$$
\mathfrak{D}\left(A, \frac{\pi}{2}\right)=\mathcal{D}\left(A, \frac{\pi}{2}\right) .
$$

The systems $\mathcal{D}(A, \varepsilon), 0 \leq \varepsilon \leq \frac{\pi}{2}$, and $\mathfrak{D}\left(A, \varepsilon+\frac{\pi}{2}\right), \frac{\pi}{2} \leq \varepsilon<\infty$, which are shown in Figures 3 and 4, respectively, allow us to introduce the corresponding ordered (by inclusion $\supset$ ) systems of spaces

$$
B(R, A, \varepsilon), \quad \frac{\pi}{2} \leq \varepsilon<\infty,
$$

and

$$
W(R, A, \varepsilon), \quad \frac{\pi}{2} \leq \varepsilon<\infty,
$$

and to carry all the results presented in this section for the case where $0 \leq \varepsilon \leq \frac{\pi}{2}$ over to the case where $\frac{\pi}{2} \leq \varepsilon<\infty$.

Statement 8. Assume that $P(z) \in W(R, A, \varepsilon), 0<\varepsilon<\infty$. The function $P(z)$ encounters Stokes' phenomenon in the region $\mathcal{P}(R, \varepsilon)$ if and only if there exists $\theta, 0 \leq \theta<\varepsilon$, such that the function $F(t)$ belongs to $B\left(R, A, \frac{\pi}{2}+\theta\right)$ and has a nonempty set of finite singular points in the region $\mathfrak{D}(A, \theta)$.

In other words, $P(z)$ encounters Stokes' phenomenon in the region $\mathcal{P}(R, \varepsilon)$ if the sectorial region of analyticity of $F(t)$ is not very small, having a positive opening at $\infty$, and is also not very large, i.e., is not larger than $\mathfrak{D}(A, \varepsilon)$. 


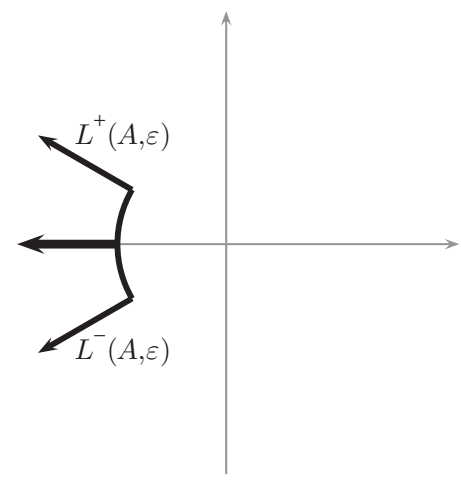

FIGURE 4. Expanding system of regions $\mathfrak{D}(A, \varepsilon), \frac{\pi}{2} \leq \varepsilon \leq \pi$, in the $t$-plane.

2.4. Discussion. Unfortunately, we cannot state that

$$
\mathcal{L} B(R, A, \varepsilon)=W(R, A, \varepsilon),
$$

where $\mathcal{L}$ is the standard Laplace transform operator. However, introducing the extensions of these spaces,

$$
\mathbf{B}(R, A, \varepsilon)=\bigcap B\left(R^{\prime}, A^{\prime}, \varepsilon^{\prime}\right)
$$

and

$$
\mathbf{W}(R, A, \varepsilon)=\bigcap W\left(R^{\prime}, A^{\prime}, \varepsilon^{\prime}\right),
$$

where the intersections are taken over all $R<R^{\prime}<\infty, A>A^{\prime}>0, \varepsilon>\varepsilon^{\prime} \geq 0$, and providing $\mathbf{B}(R, A, \varepsilon)$ and $\mathbf{W}(R, A, \varepsilon)$ with countable normalized topologies, we have the following assertion.

\section{Statement 9.}

$$
\mathcal{L} \mathbf{B}(R, A, \varepsilon)=\mathbf{W}(R, A, \varepsilon),
$$

where $\mathcal{L}$ is surjective. Moreover, the topological space $\mathbf{W}(R, A, \varepsilon)$ is isomorphic to the topological space $\mathbf{B}(R, A, \varepsilon)$.

A natural question arises: is it possible to find a version of the duality theorem which is sensitive to the boundary of the region $D(A, \varepsilon)$ ? In particular, is it possible to bring together the assumptions and the conclusions of Nevanlinna's theorem? Let us call attention to the following obstruction to such a convergence.

In [27] we presented a widest class of analytic functions satisfying (2), the elements of which are in one-to-one correspondence with their expansions. We considered a function $P(z)$ analytic in the right half-plane of the $z$-plane and such that

$$
\left|P(z)-\sum_{k=0}^{n-1} \frac{p_{k}}{z^{k+1}}\right| \leq \frac{M(\delta) n !}{(a(\delta))^{n}|z|^{n+1}}, \quad n=0,1, \ldots,
$$

in every region $\left\{z \in \mathbb{C}:-\frac{\pi}{2}+\delta<\arg z<\frac{\pi}{2}-\delta\right\}$.

It was shown that if $M(\delta)$ and $a(\delta)$ satisfy the conditions

$$
\int_{0}^{\frac{\pi}{2}} \log \log M(\delta) d \delta<\infty
$$

and

$$
\frac{\delta}{a(\delta)} \rightarrow 0 \quad \text { as } \quad \delta \rightarrow 0
$$


then $P(z)$ is uniquely determined by the coefficients of its expansion. It is possible to prove that if one of the conditions (40) or (41) is violated, then (in general) the uniqueness property fails.

We conjectured that if

$$
M(\delta)=M \exp (b / \delta),
$$

where the positive numbers $M$ and $b$ do not depend on $\delta$, and $a(\delta)=A$, then the function $F(t)$ given by (5) is analytic in $\mathcal{D}(A)$. We believe that condition (42) determines a border between analyticity and quasianalyticity. We believe also that Rezende's result [24] could shed light on this assumption.

\section{§3. Duality}

In what follows we present a roughest version of the duality theorem; a motivation for this will be explained below.

3.1. The operator $\mathcal{L} \alpha: \mathbf{B}(R, A, \varepsilon) \rightarrow \mathbf{W}(R, A, \varepsilon)$. Given $-\pi<\theta<\pi$, let

$$
l_{\theta}=\left\{z=\rho e^{i \theta}: 0<\rho<+\infty\right\} .
$$

Assume that $F(t) \in B(R, A, \varepsilon)$. Given $\alpha$ with $\operatorname{Re}\{\alpha\}>-1$, we define

$$
P(z, \alpha)=z^{\alpha} \int_{0}^{+\infty} e^{-t z} t^{\alpha} F(t) d t .
$$

Then $P(z, \alpha)$ is analytic in the region $\mathcal{P}(R)$ given by (1). Since $F(t)$ is analytic in the sectorial region $\{t \in \mathbb{C}:-\varepsilon<\arg t<\varepsilon, 0<|t|<\infty\}$, for $0 \leq|\theta|<\varepsilon$ we can also introduce the function

$$
P_{\theta}(z, \alpha)=z^{\alpha} \int_{l_{\theta}} e^{-t z} t^{\alpha} F(t) d t
$$

which is analytic in the region

$$
\mathcal{P}_{\theta}(R)=\left\{z \in \mathbb{C}:-\frac{\pi}{2}-\theta<\arg z<\frac{\pi}{2}-\theta, R<|z|<\infty\right\} .
$$

Setting $\theta=0$ gives $\mathcal{P}_{0}(R)=\mathcal{P}(R)$. Consider the region

$\mathcal{P}_{\theta}(R) \cap \mathcal{P}(R)=\left\{z \in \mathbb{C}: \max \left(-\frac{\pi}{2},-\frac{\pi}{2}+\theta\right)<\arg z<\min \left(\frac{\pi}{2}, \frac{\pi}{2}-\theta\right), R<|z|<\infty\right\}$.

Using Cauchy's theorem, for $z \in \mathcal{P}_{\theta}(R) \cap \mathcal{P}(R)$ we have

$$
z^{\alpha} \int_{0}^{+\infty} e^{-t z} t^{\alpha} F(t) d t=z^{\alpha} \int_{l_{\theta}} e^{-t z} t^{\alpha} F(t) d t, \quad 0 \leq|\theta|<\varepsilon
$$

Relations (45) show that the functions $P_{\theta}(z, \alpha), 0 \leq|\theta|<\varepsilon$, are analytic continuations of one another, so that all these functions can be viewed as elements of a single analytic function,

$$
P(z, \alpha)=P_{\theta}(z, \alpha), \quad z \in \mathcal{P}_{\theta}(R), \quad 0 \leq|\theta| \leq \varepsilon,
$$

and for this function we reserve the notation given by (44). So, by the analytic continuation principle, $P(z, \alpha)$ is analytic in the region $\mathcal{P}(R, \varepsilon)$ given by ([6).

Given arbitrary $\alpha, \operatorname{Re}\{\alpha\}>-1$, let the Laplace transformation operator $\mathcal{L}_{\alpha}$ be defined on $B(R, a, \varepsilon)$ as

$$
\mathcal{L}_{\alpha}\{F\}(z)=P(z, \alpha), \quad F \in B(R, a, \varepsilon),
$$

where $P(z, \alpha)$ is given by (46). 
3.2. The duality theorem. The following theorem holds.

Theorem 1 (Duality theorem). For every $\alpha, \operatorname{Re}\{\alpha\}>-1$, we have

$$
\mathcal{L}_{\alpha} \mathbf{B}(R, A, \varepsilon)=\mathbf{W}(R, A, \varepsilon),
$$

and $\mathcal{L}_{\alpha}$ is surjective. Moreover, the topological space $\mathbf{W}(R, A, \varepsilon)$ is isomorphic to the topological space $\mathbf{B}(R, A, \varepsilon)$.

The presence of many isomorphisms $\mathcal{L}_{\alpha}, \operatorname{Re}\{\alpha\}>-1$, between two dual spaces opens up the question of the relationship between two such operators and, in its turn, suggests the existence of a general theory of parametric representations, the existence of which would be important for applications. Many specific instances for special functions, which suggest such a general theory, were discussed in Buchholz [28].

The space $\mathbf{B}(R, A, \varepsilon)$ is closed under convolution and differentiation. An immediate application of Theorem 1 is that these structures can be transferred from $\mathbf{B}(R, A, \varepsilon)$ to $\mathbf{W}(R, A, \varepsilon)$. Thus, the spaces $\mathbf{W}(R, A, \varepsilon)$ are closed under multiplication and the left "shift" operator $S_{l}$. Since every element of the space $\mathbf{W}(R, A, \varepsilon)$ is uniquely determined by a sequence $\left\{p_{0}, p_{1}, \ldots\right\}$ satisfying (2), the operator $S_{l}$ is defined by $S_{l}\left\{p_{0}, p_{1}, \ldots\right\}=$ $\left\{p_{1}, p_{2}, \ldots\right\}$.

Indeed, Theorem 1 opens up the possibility of creating a version of the operational calculus for the spaces $\mathbf{W}(R, A, \varepsilon)$.

An analytic function $f$ operates on $\mathbf{W}(R, A, \varepsilon)$ if $P(z) \in \mathbf{W}(R, A, \varepsilon)$ implies that $f(P) \in \mathbf{W}(R, A, \varepsilon)$.

We can expect that the function exp operates on $\mathbf{W}(R, A, \varepsilon)$.

Our main purpose in this paper is to develop a technique for the proof of Theorem 1.

Let us study the following two operators generated by $\mathcal{L}_{\alpha}$.

Setting $\mathcal{L}=\mathcal{L}_{0}$, where $\mathcal{L}_{0}$ is the standard Laplace transformation operator, we introduce two automorphisms:

$$
\mathcal{D}_{\alpha}=\mathcal{L}_{\alpha} \mathcal{L}^{-1}: \mathbf{W}(R, A, \varepsilon) \rightarrow \mathbf{W}(R, A, \varepsilon)
$$

and

$$
\mathcal{T}_{\alpha}=\mathcal{L}^{-1} \mathcal{L}_{\alpha}: \mathbf{B}(R, A, \varepsilon) \rightarrow \mathbf{B}(R, A, \varepsilon) .
$$

From Theorem 1 it follows that

$$
\mathcal{D}_{\alpha} \mathbf{W}(R, A, \varepsilon)=\mathbf{W}(R, A, \varepsilon)
$$

and

$$
\mathcal{T}_{\alpha} \mathbf{B}(R, A, \varepsilon)=\mathbf{B}(R, A, \varepsilon)
$$

and that $\mathcal{D}_{\alpha}$ and $\mathcal{T}_{\alpha}$ can be viewed as dual automorphisms.

It is worth noting that the operator $\mathcal{D}_{\alpha}$ can be regarded as a version of the fractional derivative of order $\alpha$ for elements of $\mathbf{W}(R, A, \varepsilon)$.

In what follows, we derive integral representations for both of these operators. It will be shown that the kernels of these integral representations for $\mathcal{D}_{\alpha}$ and $\mathcal{T}_{\alpha}$ possess some remarkable properties, which form the basis of the technique described below.

Finally, it should be noted that our Theorem 1 is the weakest version of a duality theorem. In particular, it is insensitive to the behavior of $F(t) \in B(R, A, \varepsilon)$ on the boundary $\partial \mathcal{D}(A, \varepsilon)$ of the region $\mathcal{D}(A, \varepsilon)$.

A further improvement of this theory, which might include effects related to the properties of the elements of $B(R, A, \varepsilon)$ on the boundary, for example, distinguish cases in which $F(t)$ is bounded from those in which $F(t)$ has poles on $\partial \mathcal{D}(A, \varepsilon)$, requires finer techniques, which are not available at present. 


\section{§4. Parametric integral Representations FOR LAPLACE-MELLIN TRANSFORMS}

In order to prove the duality theorem, we need to investigate the remainder $\mathrm{P}_{n}(z)$ as a function of two variables $z$ and $n$. It is reasonable to replace $n$ by a continuous and even complex parameter. The simplest way to do this is to consider $\mathrm{P}_{n}(z, \alpha)$. Then we shall see in $\S 5$ how the parameter $n$ is replaced by $\alpha+n$, which allows us to study $\mathrm{P}_{n}(z, \alpha)$ as an analytic function of two variables $z$ and $\alpha+n$. Taking formula (26) into account, first we provide a similar study for the function $F(t, \alpha)$. We have mentioned above that this study is of independent interest.

We begin with proving an integral representation theorem for $F(t, \alpha)$.

Assume that $F(t) \in \mathbf{B}(R, A, \varepsilon)$. Given $\alpha, \operatorname{Re}\{\alpha\}>-1$, the function $z^{-\alpha} P(z, \alpha)$ (see (46) ) can be viewed as the Laplace transform of the function $t^{\alpha} F(t)$. On the other hand, for $-\frac{\pi}{2}<\arg z<\frac{\pi}{2}$, this function can be viewed as the Mellin transform of $e^{-t z} F(t)$. Next, $P(z, \alpha)$ can also be represented in the form

$$
P(z, \alpha)=\mathcal{D}_{\alpha} P(z)
$$

where $P(z)$ is the standard Laplace transform of $F(t), P=\mathcal{L} F$, and $\mathcal{D}_{\alpha}$ is given by (49). Thus, $P(z, \alpha)$ can also be viewed as a version of the fractional derivative of $P(z)$ of order $\alpha$.

The function $F(t)$ is analytic in the disk

$$
D(A)=\{t \in \mathbb{C}:|t|<A\}
$$

and can be represented there by its Taylor series

$$
F(t)=\sum_{k=0}^{\infty} f_{k} t^{k}
$$

It follows that

$$
P(z, \alpha) \sim \sum_{k=0}^{\infty} \frac{p_{k}(\alpha)}{z^{k+1}}, \quad z \in \mathcal{P}(R, \varepsilon), \quad z \rightarrow \infty
$$

where

$$
p_{k}(\alpha)=f_{k} \Gamma(\alpha+k+1) .
$$

Now we introduce the function $F(t, \alpha)$ as follows:

$$
F(t, \alpha)=\mathcal{T}_{\alpha}\{F(t)\}=\mathcal{L}^{-1} \mathcal{L}_{\alpha} F(t),
$$

where $\mathcal{T}_{\alpha}$ is given by (50), and a simple argument shows that, in the disk $D(A), F(t, \alpha)$ can be represented in the form

$$
F(t, \alpha)=\sum_{k=0}^{\infty} f_{k}(\alpha) t^{k}
$$

where

$$
f_{k}(\alpha)=f_{k} \frac{\Gamma(\alpha+k+1)}{k !}
$$

and the $f_{k}$ are given by (52). It follows that the series (56) is absolutely convergent in $D(A)$.

Our major goal in this section is to show that $F(t, \alpha)$ admits analytic continuation from $D(A)$ to the region $\mathcal{D}(A, \varepsilon)$ given by (18), and that $F(t, \alpha)$ is an element of $\mathbf{B}(R, A, \varepsilon)$. 
Remark 2. The relation

$$
P(z, \alpha)=\frac{p_{0}(\alpha)}{z}\left(1+O\left(\frac{1}{z}\right)\right), \quad z \in \mathcal{P}(R, \varepsilon), \quad z \rightarrow \infty,
$$

follows from (53) for $\varepsilon>0$. This means that for every $\delta, 0<\delta<\varepsilon$, there exists a positive constant $M_{\delta}$ such that if $|\arg z|<\frac{\pi}{2}+\varepsilon-\delta$, then

$$
\left|P(z, \alpha)-\frac{p_{0}(\alpha)}{z}\right|<\frac{M_{\delta}}{|z|^{2}} .
$$

Furthermore, it follows that $P(z, \alpha)$ is a square integrable function on all lines

$$
\{z: \operatorname{Re} z=r, R<r<\infty\}
$$

which provides analytic continuation of $F(t, \alpha)$ to the regions $\mathcal{D}\left(A, \varepsilon^{\prime}\right), 0<\varepsilon^{\prime}<\varepsilon$.

However, this argument only works if $\varepsilon>0$, and this is a principal drawback of the Poincaré approach.

4.1. An integral representation for $F(t, \alpha)$. Assume that $0 \leq R<\infty, 0<A<\infty$, and $0 \leq \varepsilon \leq \frac{\pi}{2}$.

Theorem 2. Let $F(t) \in \mathbf{B}(R, A, \varepsilon)$ be given, and let, for fixed $\operatorname{Re}\{\alpha\}>-1$, the function $F(t, \alpha)$ be defined by (56) and (57). Then:

(i) For any $r, 0 \leq R<r<\infty$, and for $t \in \mathcal{D}(A, \varepsilon)$, the following integral representation is valid:

$$
F(t, \alpha)=\sum_{k=0}^{\infty} \frac{\Gamma(\alpha+k+1)}{(k !)^{2}}(r t)^{k} f_{k}(\alpha, r, t)
$$

where

$$
f_{k}(\alpha, r, t)=\frac{1}{2 \pi i} \int_{\gamma^{\prime}}\left({ }_{2} F_{1}\left(\alpha+k+1,1 ; k+1 ; \frac{t}{\xi}\right)\right) \frac{F(\xi) e^{-r \xi} d \xi}{\xi},
$$

and $\left({ }_{2} F_{1}\left(\alpha+k+1,1 ; k+1 ; \frac{t}{\xi}\right)\right)$ is the hypergeometric function. The path $\gamma^{\prime}=\gamma\left(A^{\prime}, \varepsilon\right)$, see (19), is oriented in the counterclockwise direction, where $A^{\prime}$ satisfies $0<A^{\prime}<A$ and $t$ belongs to $\mathcal{D}\left(A^{\prime}, \varepsilon\right)$.

(ii) For $0<A^{\prime}<A$, the integral (59) and the sum (58) are absolutely convergent for $t \in \mathcal{D}(A, \varepsilon)$, and $F(t, \alpha)$ admits analytic continuation from the disk $D(A)$ to the region $\mathcal{D}(A, \varepsilon)$.

(iii) We have

$$
F(t, \alpha) \in \mathbf{B}(R, A, \varepsilon)
$$

and

$$
P(z, \alpha)=\mathcal{L} F(t, \alpha)
$$

where $P(z, \alpha)$ is given by (44).

Corollary 1. If for some $\alpha$ with $\operatorname{Re}\{\alpha\}>-1$, relation (60) is valid, then for all $\operatorname{Re}\{\beta\}>-1$ we have

$$
F(t, \beta) \in \mathbf{B}(R, A, \varepsilon) .
$$

Remark 3. For the special case where $F(t)$ satisfies all the assumptions of Theorem 2, and $\frac{1}{t} F(t)$ is absolutely integrable along the contour $\gamma=\gamma(A, \varepsilon)$, the representation of Theorem 2 can be simplified significantly to read

$$
F(t, \alpha)=\frac{\Gamma(\alpha+1)}{2 \pi i} \int_{\gamma} \frac{1}{\left(1-\frac{t}{\xi}\right)^{\alpha+1}} \cdot \frac{F(\xi) d \xi}{\xi} .
$$


This case is the subject of a separate investigation; see [29].

Proof. (i) Since $r>R$, condition (20) allows us to represent $F(t)$ in the form

$$
F(t)=\frac{1}{2 \pi i} e^{r t} \int_{\gamma^{\prime}} \frac{F(\xi) e^{-r \xi} d \xi}{\xi-t},
$$

where

$$
t \in \mathcal{D}\left(A^{\prime}, \varepsilon\right), \quad \gamma^{\prime}=\gamma\left(A^{\prime}, \varepsilon\right), \quad 0<A^{\prime}<A,
$$

and the integral is absolutely convergent for all $t \in \mathcal{D}\left(A^{\prime}, \varepsilon\right)$.

Using this representation, we evaluate the Taylor coefficients $f_{k}, k=1,2, \ldots$, of $F(t)$. The relation

$$
\left(e^{r t}(\xi-t)^{-1}\right)^{(k)}=\sum_{j=0}^{k} \frac{k !}{j !(k-j) !} r^{k-j} j !(\xi-t)^{-1-j} e^{r t},
$$

where differentiation is with respect to $t$, implies that

$$
\left.\left(e^{r t}(\xi-t)^{-1}\right)^{(k)}\right|_{t=0}=\sum_{j=0}^{k} \frac{k !}{(k-j) !} r^{k-j} \frac{1}{\xi^{1+j}},
$$

and from (63) we derive the following expression for the coefficients:

$$
f_{k}=\frac{F^{(k)}(0)}{k !}=\frac{1}{2 \pi i} \sum_{j=0}^{k} \frac{1}{(k-j) !} r^{k-j} \int_{\gamma^{\prime}} \frac{F(\xi) e^{-r \xi} d \xi}{\xi^{1+j}} .
$$

Relation (57) shows that the function $F(t, \alpha)$ given by (55) can be represented as

$$
F(t, \alpha)=\sum_{k=0}^{\infty} \frac{\Gamma(\alpha+k+1)}{k !} f_{k} t^{k},
$$

and the series is absolutely convergent for $t \in D(A)$. Substituting the expression for $f_{k}$ from (65) into (66) yields

$$
F(t, \alpha)=\frac{1}{2 \pi i} \sum_{k=0}^{\infty} \frac{\Gamma(\alpha+k+1)}{k !} t^{k} \sum_{j=0}^{k} \frac{1}{(k-j) !} r^{k-j} \int_{\gamma^{\prime}} \frac{F(\xi) e^{-r \xi} d \xi}{\xi^{1+j}} .
$$

Using the following series of transformations,

$$
\begin{aligned}
& F(t, \alpha)=\frac{1}{2 \pi i} \sum_{j=0}^{\infty} \sum_{k=j}^{\infty} \frac{\Gamma(\alpha+k+1)}{k !} t^{k} \frac{1}{(k-j) !} r^{k-j} \int_{\gamma^{\prime}} \frac{F(\xi) e^{-r \xi} d \xi}{\xi^{1+j}} \\
& \sum_{k=j}^{\infty} \frac{\Gamma(\alpha+k+1)}{k !} \frac{1}{(k-j) !} t^{k} r^{k-j}=\sum_{k=0}^{\infty} \frac{\Gamma(\alpha+k+j+1)}{(k+j) !} \frac{1}{k !} t^{k+j} r^{k} \\
& F(t, \alpha)=\frac{1}{2 \pi i} \sum_{j=0}^{\infty} \sum_{k=0}^{\infty} \frac{\Gamma(\alpha+k+j+1)}{(k+j) !} \frac{1}{k !} t^{k+j} r^{k} \int_{\gamma^{\prime}} \frac{F(\xi) e^{-r \xi} d \xi}{\xi^{1+j}}
\end{aligned}
$$

we obtain

$$
F(t, \alpha)=\frac{1}{2 \pi i} \sum_{k=0}^{\infty} \frac{r^{k} t^{k}}{k !} \sum_{j=0}^{\infty} \frac{\Gamma(\alpha+k+j+1)}{(k+j) !} t^{j} \int_{\gamma^{\prime}} \frac{F(\xi) e^{-r \xi} d \xi}{\xi^{1+j}} .
$$

The identity

$$
\sum_{j=0}^{\infty} \frac{\Gamma(\alpha+k+j+1)}{(k+j) !} t^{j}=\frac{\Gamma(\alpha+k+1)}{k !} \sum_{j=0}^{\infty} \frac{(\alpha+k+1)_{j}(1)_{j}}{(k+1)_{j} j !} t^{j}
$$


allows us to rewrite (67) as follows:

(69)

$$
F(t, \alpha)=\frac{1}{2 \pi i} \sum_{k=0}^{\infty} \frac{\Gamma(\alpha+k+1)}{(k !)^{2}}(r t)^{k} \int_{\gamma^{\prime}}\left({ }_{2} F_{1}\left(\alpha+k+1,1 ; k+1 ; \frac{t}{\xi}\right)\right) \frac{F(\xi) e^{-r \xi} d \xi}{\xi} .
$$

(ii) In part (i) we derived an integral representation of $F(t, \alpha)$ for $t \in D(A)$.

To extend the integral representation (69) to the entire region $\mathcal{D}(A, \varepsilon)$, we consider separately the $k$ th term of the sum (69),$k \in \mathbb{Z}^{+}$.

For $k=0$ we have

$$
\frac{\Gamma(\alpha+1)}{2 \pi i} \int_{\gamma^{\prime}}\left({ }_{2} F_{1}\left(\alpha+1,1 ; 1 ; \frac{t}{\xi}\right)\right) \frac{F(\xi) e^{-r \xi} d \xi}{\xi},
$$

which can be rewritten, by using (15.1.8) of [31], in the form

$$
\frac{\Gamma(\alpha+1)}{2 \pi i} \int_{\gamma^{\prime}} \frac{1}{\left(1-\frac{t}{\xi}\right)^{\alpha+1}} \frac{F(\xi) e^{-r \xi} d \xi}{\xi},
$$

as in Remark 3.

To investigate the other terms of (69), for $k \in \mathbb{N}$, we need the following lemma.

Lemma 1. Let $t$ be fixed, and assume that $t \in \mathcal{D}\left(A^{\prime}\right)$ for some $A^{\prime}, 0<A^{\prime}<A$. Assume further that $\xi \in \gamma(A), k \in \mathbb{N}$, and $\alpha$ is a complex number such that $\operatorname{Re}\{\alpha\}>-1$. Then the function ${ }_{2} F_{1}\left(\alpha+k+1,1 ; k+1 ; \frac{t}{\xi}\right)$ can be represented in the form

$$
{ }_{2} F_{1}\left(\alpha+k+1,1 ; k+1 ; \frac{t}{\xi}\right)=\frac{k}{\left(1-\frac{t}{\xi}\right)^{\alpha+1}} \int_{0}^{1} v^{k-1}\left(1-\frac{t}{\xi} v\right)^{\alpha} d v .
$$

Moreover, there exists a positive constant $M=M\left(\alpha, A^{\prime}\right)$ independent of $k$ and such that

$$
\left|{ }_{2} F_{1}\left(\alpha+k+1,1 ; k+1 ; \frac{t}{\xi}\right)\right| \leq \frac{k}{\sqrt{2 k-1}} M\left(\alpha, A^{\prime}\right)\left|\left(\frac{\xi}{\xi-t}\right)^{\alpha+1}\right| .
$$

Proof. It is known that if $\operatorname{Re}\{c\}>\operatorname{Re}\{b\}>0$, then the Euler representation for the hypergeometric function,

$$
{ }_{2} F_{1}(a, b ; c ; x)=\frac{\Gamma(c)}{\Gamma(b) \Gamma(c-b)} \int_{0}^{1} \tau^{b-1}(1-\tau)^{c-b-1}(1-x \tau)^{-a} d \tau,
$$

is valid in the complex $x$-plane cut along the interval $(1,+\infty)$; see, for example, 30 , Theorem 2.2.1].

The conditions $\xi \in \gamma(A)$ and $t \in \mathcal{D}\left(A^{\prime}\right)$ ensure that

$$
\frac{t}{\xi} \notin(1,+\infty)
$$

so that, with $k \in \mathbb{N}$, we can apply (74) to the kernel of (69) (the graph of $\frac{t}{\xi}$ with $\xi$ running along the boundary $\gamma(A)$, for $t=3 A+0.9 i A$, is shown in Figure 5).

Thus, we have

$$
{ }_{2} F_{1}\left(\alpha+k+1,1 ; k+1 ; \frac{t}{\xi}\right)=k \int_{0}^{1} \frac{(1-\tau)^{k-1}}{\left(1-\frac{t}{\xi} \tau\right)^{\alpha+k+1}} d \tau .
$$

Set

$$
x=\frac{t}{\xi}-1
$$




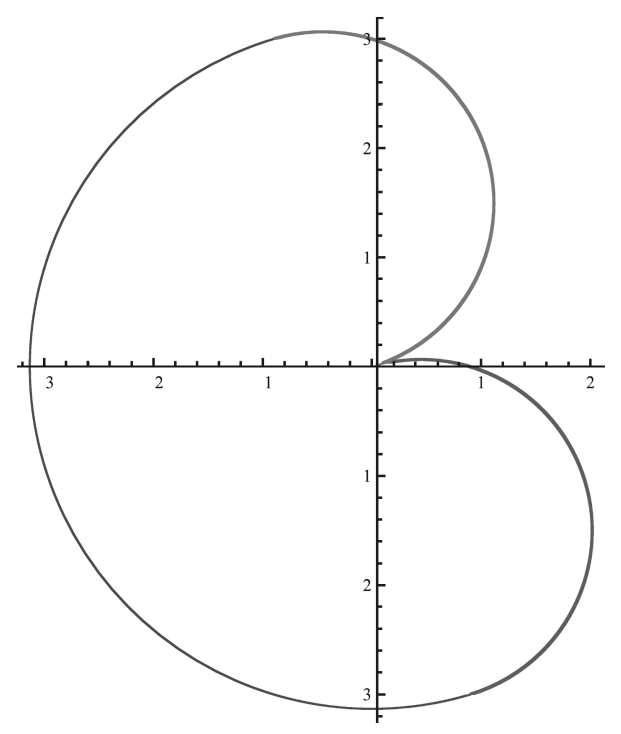

Figure 5. The graph of $\frac{t}{\xi}$ when $\xi$ runs along the boundary $\gamma(A)$, for $t=3 A+0.9 i A$.

so that $x \notin(0,+\infty)$. We make a series of transformations of the integral:

$$
\begin{aligned}
I(x) & =\int_{0}^{1} \frac{(1-\tau)^{k-1}}{\left(1-\frac{t}{\xi} \tau\right)^{\alpha+k+1}} d \tau=\int_{0}^{1} \frac{(1-\tau)^{k-1}}{(1-\tau-x \tau)^{\alpha+k+1}} d \tau \\
& =\int_{0}^{1} \frac{(1-\tau)^{k-1}}{(1-\tau-x \tau)^{k-1}} \cdot \frac{1}{(1-\tau-x \tau)^{\alpha+2}} d \tau \\
& =\int_{0}^{1} \frac{\left(\frac{1-\tau}{\tau}\right)^{k-1}}{\left(\frac{1-\tau}{\tau}-x\right)^{k-1}} \cdot \frac{1}{\tau^{\alpha+2}\left(\frac{1-\tau}{\tau}-x\right)^{\alpha+2}} d \tau .
\end{aligned}
$$

Letting

$$
\frac{1-\tau}{\tau}=s, \text { whence } \tau=\frac{1}{s+1} \text { and } d \tau=-\frac{d s}{(s+1)^{2}}
$$

we have

$$
I(x)=\int_{0}^{\infty} \frac{s^{k-1}}{(s-x)^{k-1}} \cdot \frac{(s+1)^{\alpha+2}}{(s-x)^{\alpha+2}} \cdot \frac{d s}{(s+1)^{2}},
$$

which can be rewritten as

$$
I(x)=\int_{0}^{\infty}\left(\frac{s}{s-x}\right)^{k-1} \cdot(s+1)^{\alpha}\left(\frac{s}{s-x}\right)^{\alpha+2} \cdot \frac{d s}{s^{\alpha+2}} .
$$

Using the substitution

$$
\frac{s}{s-x}=v
$$

so that

$$
s=-x \frac{v}{1-v}, \quad d s=\frac{-x d v}{(1-v)^{2}}, \quad s+1=\frac{1-v-x v}{1-v},
$$

we can assume without serious restrictions that $\operatorname{Im}\{x\} \neq 0$. In fact, when $\xi$ runs along the boundary $\gamma(A)$, there are exactly two real values of $x$, see Figure 5 , and in such a situation we can use Cauchy's theorem and deform the integration path to be away from the dangerous value of $x$ keeping the endpoints fixed. 
If $x$ is complex, then the image of the real line in the $s$-plane is a circle $C$ in the $v$-plane. Since

$$
s=0,1, \infty \Longleftrightarrow v=0, \frac{1}{1-x}, 1, \quad \text { respectively, }
$$

the image of the positive ray in the $s$-plane is the $\operatorname{arc}\left(0, \frac{1}{1-x}, 1\right)$ of $C$. Then we have

$$
I(x)=\int_{C\left(0, \frac{1}{1-x}, 1\right)} v^{k-1}\left(\frac{1-v-x v}{1-v}\right)^{\alpha} v^{\alpha+2} \frac{-x d v}{\left(-x \frac{v}{1-v}\right)^{\alpha+2}(1-v)^{2}},
$$

which can be rewritten as

$$
I(x)=\frac{1}{(-x)^{\alpha+1}} \int_{C\left(0, \frac{1}{1-x}, 1\right)} v^{k-1}(1-v-x v)^{\alpha} \cdot d v .
$$

The only singular point of the integrand in (77) is

$$
v=\frac{1}{1+x} \text {. }
$$

Since

$$
s=-1 \Longleftrightarrow v=\frac{1}{1+x},
$$

this singular point belongs to the supplementary arc $\left(0, \frac{1}{1+x}, 1\right)$ of $C$.

Using Cauchy's theorem, we can deform the arc $\left(0, \frac{1}{1-x}, 1\right)$ to the interval $(0,1)$ on the positive ray of the $v$-plane without crossing the supplementary arc $\left(0, \frac{1}{1+x}, 1\right)$. Then the integral (77) can be rewritten as

$$
I(x)=\frac{1}{(-x)^{\alpha+1}} \int_{0}^{1} v^{k-1}(1-v-x v)^{\alpha} d v
$$

so that

$$
{ }_{2} F_{1}\left(\alpha+k+1,1 ; k+1 ; \frac{t}{\xi}\right)=\frac{k}{\left(1-\frac{t}{\xi}\right)^{\alpha+1}} \int_{0}^{1} v^{k-1}\left(1-\frac{t}{\xi} v\right)^{\alpha} d v .
$$

This proves (72).

Now, we use the Cauchy-Bunyakovskiu inequality to obtain

$$
\begin{aligned}
& \left|{ }_{2} F_{1}\left(\alpha+k+1,1 ; k+1 ; \frac{t}{\xi}\right)\right| \\
& \quad \leq \frac{k}{\left|\left(1-\frac{t}{\xi}\right)^{\alpha+1}\right|}\left(\int_{0}^{1} v^{2 k-2} d v\right)^{\frac{1}{2}} \cdot\left(\int_{0}^{1}\left|\left(1-\frac{t}{\xi} v\right)^{\alpha+1}\right|^{2} d v\right)^{\frac{1}{2}},
\end{aligned}
$$

and the last factor does not depend on $k$. Setting

$$
M\left(\alpha, A^{\prime}\right)=\left(\int_{0}^{1}\left|\left(1-\frac{t}{\xi} v\right)^{\alpha+1}\right|^{2} d v\right)^{\frac{1}{2}}<\infty,
$$

we see that inequality (80) together with (79) gives (73). Thus, (i) is proved.

(iii) Using (73), we show that for every $R<r<\infty$ and every $0<A^{\prime}<A$ we have

$$
F(t, \alpha) \in B(r, A, \varepsilon) \text {, }
$$

and (60) follows from (37). 
In the next subsection we show that the function $F(t, \alpha)$ given by (55) with $F(t) \in$ $\mathbf{B}(R, A, \varepsilon)$ admits analytic continuation to the entire complex $\alpha$-plane except for simple poles at $\alpha=-n, n=1,2, \ldots$ Next, we show that every point $\alpha=-n$ generates a polynomial of degree $n$, and we provide explicit expressions for the coefficients of this polynomial.

\subsection{Analytic continuation of $F(t, \alpha)$ to the $\alpha$-plane.}

Theorem 3. Let $F(t) \in \mathbf{B}(R, A, \varepsilon)$, and let $F(t, \alpha)$ be defined as in Theorem 2. Then, for any fixed $t \in \mathcal{D}(A, \varepsilon)$,

(i) the function $F(t, \alpha)$ admits analytic continuation to the entire $\alpha$-plane except for the negative integers;

(ii) the function $F(t, \alpha)$ has a simple pole at every point $\alpha=-n-1, n=0,1, \ldots$, so that the function

$$
\frac{F(t, \alpha)}{\Gamma(\alpha+1)}
$$

is an entire function of exponential type, and

$$
\lim _{\alpha \rightarrow-n-1} \frac{F(t, \alpha)}{\Gamma(\alpha+1)}=\Phi_{n, F}(t) ;
$$

(iii) the function $\Phi_{n, F}(t)$ is a polynomial in $t$ of degree $n$, representable in the form

$$
\Phi_{n, F}(t)=\sum_{j=0}^{n}(-1)^{j}\left(\begin{array}{l}
n \\
j
\end{array}\right) f_{j} t^{j},
$$

where the coefficients $f_{j}$, given by (52), can be written as

$$
f_{j}=\frac{1}{2 \pi i} \sum_{s=0}^{j} \frac{1}{(j-s) !} r^{j-s} \int_{\gamma^{\prime}} \frac{F(\xi) e^{-r \xi} d \xi}{\xi^{1+s}} .
$$

Proof. Before proving this theorem in general, we consider the particular case where $F(t, \alpha)$ can be represented in the form (62) given in Remark 2. Statements (i) and (ii) follow immediately from this representation.

We introduce the function

$$
\Phi_{F}(t, \alpha)=\frac{F(t, \alpha)}{\Gamma(\alpha+1)} .
$$

Clearly, $\Phi_{F}(t, \alpha), t \in \mathcal{D}\left(A^{\prime}, \varepsilon\right)$, is an entire function in $\alpha$ of a finite exponential type. Then, using (81), we have

$$
\Phi_{n, F}(t)=\frac{1}{2 \pi i} \int_{\gamma^{\prime}}\left(1-\frac{t}{\xi}\right)^{n} \frac{F(\xi) d \xi}{\xi}
$$

which can be rewritten as

$$
\Phi_{n, F}(t)=\sum_{j=0}^{n}(-1)^{j}\left(\begin{array}{l}
n \\
j
\end{array}\right) t^{j} \cdot \frac{1}{2 \pi i} \int_{\gamma^{\prime}} \frac{F(\xi) d \xi}{\xi^{j+1}},
$$

and finally,

$$
\Phi_{n, F}(t)=\sum_{j=0}^{n}(-1)^{j}\left(\begin{array}{l}
n \\
j
\end{array}\right) f_{j} t^{j},
$$

where the $f_{j}$ are given by (52). 
(i)-(ii) Considering the general case, we introduce the function

$$
\Phi_{F}(t, \alpha)=\Phi(t, \alpha)=\frac{F(t, \alpha)}{\Gamma(\alpha+1)} .
$$

Using the relation

$$
\frac{\Gamma(\alpha+k+1)}{\Gamma(\alpha+1)}=(\alpha+1)_{k}=(\alpha+1)(\alpha+2) \cdots(\alpha+k), \quad k \in \mathbb{Z}^{+},
$$

and formulas (58) and (59), we have

$$
\Phi(t, \alpha)=\sum_{k=0}^{\infty} \frac{(\alpha+1)_{k}}{(k !)^{2}}(r t)^{k} f_{k}(\alpha, r, t),
$$

so that

$$
\Phi(t, \alpha)=f_{0}(\alpha, r, t)+\sum_{k=1}^{\infty} \frac{(\alpha+1)_{k}}{(k !)^{2}}(r t)^{k} f_{k}(\alpha, r, t) .
$$

From (71), (72), and (59) we obtain

$$
f_{0}(\alpha, r, t)=\frac{1}{2 \pi i} \int_{\gamma^{\prime}} \frac{1}{\left(1-\frac{t}{\xi}\right)^{\alpha+1}} \frac{F(\xi) d \xi}{\xi}
$$

and

$$
f_{k}(\alpha, r, t)=\frac{k}{2 \pi i} \int_{0}^{1} v^{k-1} d v \int_{\gamma^{\prime}} \frac{1}{1-\frac{t}{\xi}}\left(\frac{1-\frac{t}{\xi} v}{1-\frac{t}{\xi}}\right)^{\alpha} \frac{F(\xi) e^{-r \xi} d \xi}{\xi}, \quad k \in \mathbb{N},
$$

and for fixed $t$ both functions $f_{0}(\alpha, r, t)$ and $f_{k}(\alpha, r, t), k \in \mathbb{N}$, are entire functions of $\alpha$ of exponential type.

(iii) Given $n \in \mathbb{N}$, we evaluate

$$
\Phi(t,-n-1)=\sum_{k=0}^{\infty} \frac{(-n)_{k}}{(k !)^{2}}(r t)^{k} f_{k}(-n-1, r, t) .
$$

Since

$$
{ }_{2} F_{1}(-n+k+1,1 ; k+1 ; x) \equiv 0
$$

for $k \geq n+1$, so that

$$
\sum_{k=n+1}^{\infty} \frac{(-n)_{k}}{(k !)^{2}}(r t)^{k} f_{k}(-n-1, r, t) \equiv 0
$$

relation (87) yields

$$
\Phi(t,-n-1)=\sum_{k=0}^{n} \frac{(-1)^{k} n !}{(k !)^{2}(n-k) !}(r t)^{k} f_{k}(-n-1, r, t),
$$

where

$$
f_{k}(-n-1, r, t)=\frac{1}{2 \pi i} \int_{\gamma^{\prime}}\left({ }_{2} F_{1}\left(-n+k, 1 ; k+1 ; \frac{t}{\xi}\right)\right) \frac{F(\xi) e^{-r \xi} d \xi}{\xi} .
$$

Using the relations

$$
{ }_{2} F_{1}\left(-n+k, 1 ; k+1 ; \frac{t}{\xi}\right)=\sum_{j=0}^{n-k} \frac{(-n+k){ }_{j}(1)_{j}}{(k+1)_{j} j !}\left(\frac{t}{\xi}\right)^{j}
$$

and

$$
\sum_{j=0}^{n-k} \frac{(-n+k)_{j}(1)_{j}}{(k+1)_{j} j !}=\sum_{j=0}^{n-k} \frac{(-1)^{j}(n-k) ! k !}{(n-k-j) !(k+j) !},
$$


we can rewrite the expression (89) as

$$
f_{k}(-n-1, r, t)=\sum_{j=0}^{n-k} \frac{(-1)^{j}(n-k) ! k !}{(n-k-j) !(k+j) !} t^{j}\left(\frac{1}{2 \pi i} \int_{\gamma^{\prime}} \frac{F(\xi) e^{-r \xi} d \xi}{\xi^{1+j}}\right) .
$$

Combining (88) and (90), we get

$\Phi(t,-n-1)=\sum_{k=0}^{n} \frac{(-1)^{k} n !}{(k !)^{2}(n-k) !}(r t)^{k} \sum_{j=0}^{n-k} \frac{(-1)^{j}(n-k) ! k !}{(n-k-j) !(k+j) !} t^{j}\left(\frac{1}{2 \pi i} \int_{\gamma^{\prime}} \frac{F(\xi) e^{-r \xi} d \xi}{\xi^{1+j}}\right)$,

which can be rewritten as

$$
\Phi(t,-n-1)=\sum_{k=0}^{n} \frac{n !}{k !} r^{k} \sum_{j=0}^{n-k} \frac{(-1)^{k+j}}{(n-k-j) !(k+j) !} t^{k+j}\left(\frac{1}{2 \pi i} \int_{\gamma^{\prime}} \frac{F(\xi) e^{-r \xi} d \xi}{\xi^{1+j}}\right) .
$$

The substitution $k+j=p$ yields

$$
\Phi(t,-n-1)=\sum_{p=0}^{n} \frac{(-1)^{p} n !}{(n-p) ! p !} t^{p} \sum_{j=0}^{p} \frac{1}{(p-j) !} r^{p-j}\left(\frac{1}{2 \pi i} \int_{\gamma^{\prime}} \frac{F(\xi) e^{-r \xi} d \xi}{\xi^{1+j}}\right) .
$$

Now, it remains to use (65) to obtain (82).

We return to the representation (44) for $P(z, \alpha)$.

It follows that for an arbitrary parameter $\lambda, \operatorname{Re}\{\lambda\}>-1$, the function $P(z, \alpha)$ can also be represented in the form

$$
P(z, \alpha)=z^{\lambda} \int_{0}^{\infty} e^{-z t} t^{\lambda} F(t, \alpha, \lambda) d t
$$

where

$$
F(t, \alpha, \lambda)=\sum_{k=0}^{\infty} f_{k} \frac{\Gamma(\alpha+k+1)}{\Gamma(\lambda+k+1)} t^{k}
$$

Theorem 2 shows that

$$
F(t, \alpha, \lambda) \in \mathbf{B}(R, A, \varepsilon) .
$$

This gives a general view of parametric integral representations in the theory of special functions; see, for example, the parametric representation for Whittaker functions in terms of Gauss hypergeometric functions given by formula (30) in [28, §5].

\subsection{Examples.}

Example 1. The confluent hypergeometric function $\mathbf{U}(\mathbf{a}, \mathbf{b} ; \mathbf{z})$. Let us apply the representation (91) to the following particular case: $P(z, \alpha)=\Gamma(a) U(a, b ; z)$, where $\alpha=a-1$ and $U(a, b, z)$ is the confluent hypergeometric function, that is, a solution of Kummer's equation, $z u^{\prime \prime}+(b-z) u^{\prime}-a u=0$, see [31, 13.2.5], which can be represented as

$$
U(a, b, z)=\frac{1}{\Gamma(a)} \int_{0}^{\infty} e^{-z t} t^{a-1}(1+t)^{b-a-1} d t .
$$

For an arbitrary parameter $\lambda, \operatorname{Re}\{\lambda\}>0$, we have

$$
U(a, b ; z)=\frac{z^{-a+\lambda}}{\Gamma(\lambda)} \int_{0}^{\infty} e^{-z t} t^{\lambda-1}\left({ }_{2} F_{1}(1+a-b ; a ; \lambda ;-t)\right) d t,
$$

where ${ }_{2} F_{1}(1+a-b ; a ; \lambda ;-t)$ is the hypergeometric function, and the ray of integration depends on $\arg z,-\frac{3 \pi}{2}<\arg z<\frac{3 \pi}{2}$.

Formula (93) is a version of [28, 30]. 
In particular, $\lambda=a$ yields (92), and $\lambda=1$ yields

$$
U(a, b, z)=z^{1-a} \int_{0}^{\infty} e^{-z t}\left({ }_{2} F_{1}(1+a-b ; a ; 1 ;-t)\right) d t .
$$

Example 2. The Airy function, a solution of Airy's differential equation, $u^{\prime \prime}-z u=0$, can be represented as

$$
\operatorname{Ai}(z)=\frac{1}{2 \sqrt{\pi}} z^{-\frac{1}{4}} \exp \left(-\frac{2}{3} z^{\frac{3}{2}}\right) \cdot P(\zeta),
$$

where, upon setting

$$
\zeta=\frac{2}{3} z^{\frac{3}{2}}
$$

we have

$$
P(\zeta)=\zeta \cdot \frac{\zeta^{-\frac{1}{6}}}{\Gamma\left(\frac{5}{6}\right)} \int_{0}^{\infty} e^{-t \zeta} t^{-\frac{1}{6}}\left(1+\frac{t}{2}\right)^{-\frac{1}{6}} d t, \quad-\frac{\pi}{2}<\arg \zeta<\frac{\pi}{2} .
$$

This is a particular case of (92) with $a=\frac{5}{6}$ and $b=\frac{5}{3}$,

$$
P(\zeta)=(2 \zeta)^{\frac{5}{6}} U\left(\frac{5}{6}, \frac{5}{3}, 2 \zeta\right) .
$$

Using (97) and (94), we get

$$
P(\zeta)=\zeta \int_{0}^{+\infty} e^{-t \zeta}\left({ }_{2} F_{1}\right)\left(\frac{1}{6}, \frac{5}{6}, 1 ;-\frac{t}{2}\right) d t .
$$

§5. Integral RePresentations For $P(z, \alpha)$ And $\mathrm{P}_{n}(z, \alpha)$

Now we turn to the function $P(z, \alpha)$ given by (44) , and to the errors or remainders

$$
\mathrm{P}_{n}(z, \alpha)=P(z, \alpha)-\sum_{k=0}^{n-1} \frac{p_{k}(\alpha)}{z^{k+1}}, \quad n=0,1, \ldots,
$$

where the coefficients $p_{n}(\alpha), n=0,1, \ldots$, are given by (154), and where we set $\mathrm{P}_{0}(z, \alpha)=$ $P(z, \alpha)$.

The representation (58) suggests the corresponding integral representations of $P(z, \alpha)$, given by (44).

Consider the function

$$
K(w, s)=\frac{1}{\Gamma(s+1)} \int_{0}^{\infty} e^{-t} t^{s} \frac{w}{t+w} d t
$$

which is well known in asymptotic analysis. This function is analytic and single-valued for $|\arg w|<\pi$ and $\operatorname{Re}\{s\}>-1$ and can also be represented as

$$
K(w, s)=\frac{w^{s+1}}{\Gamma(s+1)} \mathcal{L}_{s}\left\{\frac{1}{1+t}\right\} .
$$

It follows that, for fixed $s$ with $\operatorname{Re}\{s\}>-1, K(w, s)$ admits analytic continuation in the $w$-plane along any path not passing through the origin, and for fixed $w,|\arg w|<\pi$, admits analytic continuation to the entire $s$-plane, except for simple poles at the negative integers.

Given $0<A<\infty$ and $0 \leq \varepsilon<\frac{\pi}{2}$, we assume that $F(t)$ is defined by

$$
F(t)=e^{R t} \int_{\gamma} \frac{e^{-R \xi} d \sigma(\xi)}{\xi-t}, \quad t \in \mathcal{D}(A, \varepsilon),
$$


where $\mathcal{D}(A, \varepsilon)$ and $\gamma=\gamma(A, \varepsilon)$ are given by (18) and (19), respectively, and $\sigma$ is a measure supported on $\gamma$. We assume also that

$$
\int_{\gamma} \frac{e^{-R \xi}|d \sigma(\xi)|}{|\xi|}<M
$$

for some positive $M$; we do not exclude the case of $R=0$.

Clearly, $F(t) \in B(R, A, \varepsilon)$.

Let the function

$$
P(z, \alpha)=\mathcal{L}_{\alpha}\{F(t)\}
$$

be given by (44), and let $\mathrm{P}_{n}(z, \alpha)$ be given by (99))

Representing $\gamma=\gamma(A, \varepsilon)$ in the form (19),

$$
\gamma(A, \varepsilon)=l^{+}(A, \varepsilon) \cup C^{-}(A) \cup l^{-}(A, \varepsilon),
$$

for $z \in \mathcal{P}(R, \varepsilon)$ and $\operatorname{Re}\{s\}>-1$, we introduce the function

$$
\widetilde{K}(-\xi z, s)= \begin{cases}K(-\xi z, s) & \text { if } \xi \in C^{-}(A), \\ K\left(e^{i \pi} \xi z, s\right) & \text { if } \xi \in l^{-}(A, \varepsilon), \\ K\left(e^{-i \pi} \xi z, s\right) & \text { if } \xi \in l^{+}(A, \varepsilon) .\end{cases}
$$

Theorem 4A. Assume that in (102) we have $R=0$. Let $P(z, \alpha)$ be given by (104). Assume that

$$
z \in \mathcal{P}(0, \varepsilon)=\left\{z \in \mathbb{C}:-\frac{\pi}{2}-\varepsilon<\arg z<\frac{\pi}{2}+\varepsilon, 0<|z|<\infty\right\},
$$

and that $\alpha$ is fixed, $\operatorname{Re}\{\alpha\}>-1$. For $0 \leq \varepsilon<\frac{\pi}{2}$, let the kernel $\widetilde{K}(-\xi z, s)$ be given by (105). Then the following integral representations hold:

$$
\begin{aligned}
p_{n}(\alpha) & =\Gamma(\alpha+n+1) \int_{\gamma(A, \varepsilon)} \frac{d \sigma(\xi)}{\xi^{n+1}} \\
P(z, \alpha) & \equiv \frac{\Gamma(\alpha+1)}{z} \int_{\gamma(A, \varepsilon)} \frac{\widetilde{K}(-\xi z, \alpha) d \sigma(\xi)}{\xi},
\end{aligned}
$$

and

$$
\mathrm{P}_{n}(z, \alpha) \equiv \frac{\Gamma(\alpha+n+1)}{z^{n+1}} \int_{\gamma(A, \varepsilon)} \frac{\widetilde{K}(-\xi z, \alpha+n) d \sigma(\xi)}{\xi^{n+1}}, \quad n \in \mathbb{N},
$$

and the integrals (106), (107), and (108) are absolutely convergent.

Theorem 4B. Assume that in (102) we have $0 \leq R<\infty$. Let $P(z, \alpha)$ be given by (104), let $z \in \mathcal{P}(R, \varepsilon)$, and let $\alpha$ be fixed, $\operatorname{Re}\{\alpha\}>-1$. For $0 \leq \varepsilon<\frac{\pi}{2}$, let the kernel $\widetilde{K}(-\xi z, s)$ be given by (105). Then the following integral representations hold:

$$
p_{n}(\alpha)=\Gamma(\alpha+n+1) \sum_{k=0}^{n} \frac{R^{k}}{k !} \int_{\gamma(A, \varepsilon)} \frac{e^{-R \xi} d \sigma(\xi)}{\xi^{n+1-k}}
$$

$$
P(z, \alpha)=\sum_{k=0}^{\infty} \frac{\Gamma(\alpha+k+1)}{k !} \frac{R^{k}}{z^{k+1}} \int_{\gamma(A, \varepsilon)} \widetilde{K}(-\xi z, \alpha+k) \frac{e^{-R \xi} d \sigma(\xi)}{\xi}
$$


(iii)

$$
\begin{aligned}
\mathrm{P}_{n}(z, \alpha) \equiv & \frac{\Gamma(\alpha+n+1)}{z^{n+1}} \sum_{k=0}^{n-1} \frac{R^{k}}{k !} \int_{\gamma(A, \varepsilon)} \widetilde{K}(-z \xi, \alpha+n) \frac{e^{-R \xi} d \sigma(\xi)}{\xi^{n-k+1}} \\
& +\sum_{k=n}^{\infty} \frac{\Gamma(\alpha+k+1)}{k !} \frac{R^{k}}{z^{k+1}} \int_{\gamma(A, \varepsilon)} \widetilde{K}(-z \xi, \alpha+k) \frac{e^{-R \xi} d \sigma(\xi)}{\xi},
\end{aligned}
$$

and all the integrals and sums in (109), (110), and (111) are absolutely convergent.

Clearly, Theorem $4 \mathrm{~A}$ is a particular case of Theorem $4 \mathrm{~B}$.

We present separately another particular case of Theorem 4B, in which $\varepsilon=0$ and the contour $\gamma(A, \varepsilon)$ given by (19) is replaced by

$$
\gamma(A)=\{t \in \mathbb{C}: \operatorname{dist}\{t,(0,+\infty)\}=A\} .
$$

We recall that the contour $\gamma(A)$ is the boundary of the determinative region $\mathcal{D}(A)$ given by (3).

Theorem 4C. Assume that in (102) we have $\varepsilon=0$ and $0 \leq R<\infty$. Let $P(z, \alpha)$ be given by (104), and let $\alpha$ be fixed, $\operatorname{Re}\{\alpha\}>-1$. Then for $z \in \mathcal{P}(R)$ and $\xi \in \gamma(A)$ we have

$$
-\pi<\arg (-z \xi)<\pi,
$$

and the integral representations for $p_{n}(\alpha), P(z, \alpha)$, and $\mathrm{P}_{n}(z, \alpha)$ can be written as in (109), (110), and (111) with $\gamma(A, \varepsilon)$ and $\widetilde{K}(-z \xi, s)$ replaced by $\gamma(A)$ and $K(-z \xi, s)$, respectively.

All the integrals and sums are absolutely convergent.

The role of $K(w, s)$ in the integral representation above allows us to call this function the basic kernel.

By using (106), the representation (108) can also be written as

$$
\mathrm{P}_{n}(z, \alpha)=\frac{\int_{\gamma(A, \varepsilon)} \widetilde{K}(-\xi z, \alpha+n) \xi^{-n-1} d \sigma(\xi)}{\int_{\gamma(A, \varepsilon)} \xi^{-n-1} d \sigma(\xi)} \cdot \frac{p_{n}}{z^{n+1}},
$$

where the second factor is the first neglected term, while the first factor is referred to as the terminant; see [20].

Remark 4. Theorems 4A and 4C are particular cases of Theorem 4B. However, we presented them separately, because they are of independent interest and they are much easier to follow, and they help us to organize properly the proof of Theorem 4 .

We split the proof of Theorem 4 into three parts. First, we provide a formal derivation of (111). Our next aim is to obtain error bounds, that is, estimates of $\mathrm{P}_{n}(z, \alpha)$ in $\mathcal{P}(R, \varepsilon)$. The representations for the remainders show that, with this aim in mind, we must accomplish a similar study for the basic kernel $K(w, s)$ and for $K_{\varepsilon}(-\xi z, \alpha+n)$ with $\xi \in \gamma(A, \varepsilon)$, and $\mathcal{P}(R, \varepsilon)$.

\subsection{Formal derivation.}

Proof. (i) We apply the operator $\mathcal{L}_{\alpha}$ to both sides of (102) to obtain the relation

$$
P(z, \alpha)=z^{\alpha} \int_{l_{\theta}} e^{-z t} t^{\alpha} e^{R t} \int_{\gamma} \frac{e^{-R \xi} d \sigma(\xi)}{\xi-t} d t, \quad R<z<+\infty,
$$

which can be rewritten as

$$
P(z, \alpha)=\sum_{k=0}^{\infty} \frac{1}{k !} \frac{R^{k}}{z^{k+1}} \int_{\gamma} \frac{e^{-R \xi} d \sigma(\xi)}{\xi} \int_{l_{\theta}} e^{-t} t^{\alpha+k} \frac{\xi z}{\xi z-t} d t .
$$


By (100), the above representation can also be rewritten in terms of the basic kernels as

$$
P(z, \alpha)=\sum_{k=0}^{\infty} \frac{\Gamma(\alpha+k+1)}{k !} \frac{R^{k}}{z^{k+1}} \int_{\gamma} K(-\xi z, \alpha+k) \frac{e^{-R \xi} d \sigma(\xi)}{\xi} .
$$

In the sequel, we derive the coefficients $p_{n}(\alpha)$ of $P(z, \alpha)$ and the remainders $\mathrm{P}_{n, P}(z, \alpha)$ given by (154) and (15), respectively, directly from (114).

(ii) Rewriting (114) in the form

$$
P(z, \alpha)=\sum_{k=0}^{n-1} \frac{1}{k !} \frac{R^{k}}{z^{k+1}} I(R, \alpha, k, z)+\sum_{k=n}^{\infty} \frac{1}{k !} \frac{R^{k}}{z^{k+1}} I(R, \alpha, k, z),
$$

where

$$
I(R, \alpha, k, z)=\int_{\gamma} \frac{e^{-R \xi} d \sigma(\xi)}{\xi} \int_{l_{\theta}} e^{-t} t^{\alpha+k} \frac{\xi z}{\xi z-t} d t
$$

we use the expansion for the last factor in (116),

$$
\frac{\xi z}{\xi z-t}=\sum_{l=0}^{j} \frac{t^{l}}{(\xi z)^{l}}+\frac{\xi z t^{j+1}}{(\xi z)^{j+1}(\xi z-t)}, \quad j=0,1, \ldots,
$$

with $j=n-k-1$, to represent $I(R, \alpha, k, z)$ as a sum of two terms

$$
I(R, \alpha, k, z)=I_{1}(R, \alpha, k, n, z)+I_{2}(R, \alpha, k, n, z),
$$

where

$$
\begin{aligned}
I_{1}(R, \alpha, k, n, z) & =\sum_{l=0}^{n-k-1} \frac{1}{z^{l}} \int_{\gamma} \frac{e^{-R \xi} d \sigma(\xi)}{\xi^{l+1}} \int_{l_{\theta}} e^{-t} t^{\alpha+k+l} d t \\
& =\sum_{l=0}^{n-k-1} \frac{\Gamma(\alpha+k+l+1)}{z^{l}} \int_{\gamma} \frac{e^{-R \xi} d \sigma(\xi)}{\xi^{l+1}}
\end{aligned}
$$

and

$$
\begin{aligned}
I_{2}(R, \alpha, k, n, z) & =\int_{\gamma} \frac{e^{-R \xi} d \sigma(\xi)}{\xi} \int_{l_{\theta}} e^{-t} t^{\alpha+k} \frac{\xi z t^{n-k}}{(\xi z)^{n-k}(\xi z-t)} d t \\
& =\frac{1}{z^{n-k}} \int_{\gamma} \frac{e^{-R \xi} d \sigma(\xi)}{\xi^{n-k+1}} \int_{l_{\theta}} e^{-t} t^{\alpha+n} \frac{\xi z}{\xi z-t} d t .
\end{aligned}
$$

This allows us to rewrite (115) as

$$
\begin{aligned}
P(z, \alpha)=\sum_{k=0}^{n-1} \frac{1}{k !} \frac{R^{k}}{z^{k+1}} I_{1}(R, \alpha, k, n, z) & +\sum_{k=0}^{n-1} \frac{1}{k !} \frac{R^{k}}{z^{k+1}} I_{2}(R, \alpha, k, n, z) \\
& +\sum_{k=n}^{\infty} \frac{1}{k !} \frac{R^{k}}{z^{k+1}} I(R, \alpha, k, z) .
\end{aligned}
$$

We shall prove that the first sum in (120) is a polynomial in $\frac{1}{z}$ of degree $n$, while the other sums involve only higher powers of $\frac{1}{z}$. This shows that

$$
\sum_{k=0}^{n-1} \frac{p_{k}(\alpha)}{z^{k+1}}=\sum_{k=0}^{n-1} \frac{1}{k !} \frac{R^{k}}{z^{k+1}} I_{1}(R, \alpha, k, n, z)
$$

and

$$
\mathrm{P}_{n, P}(z, \alpha)=\sum_{k=0}^{n-1} \frac{1}{k !} \frac{R^{k}}{z^{k+1}} I_{2}(R, \alpha, k, n, z)+\sum_{k=n}^{\infty} \frac{1}{k !} \frac{R^{k}}{z^{k+1}} I(R, \alpha, k, z) .
$$


A straightforward calculation shows that the right-hand side of (121) can be rewritten as

$$
\begin{gathered}
\sum_{k=0}^{n-1} \sum_{l=0}^{n-k-1} \frac{\Gamma(\alpha+k+l+1)}{k !} \frac{R^{k}}{z^{k+l+1}} \int_{\gamma} \frac{e^{-R \xi} d \sigma(\xi)}{\xi^{l+1}} \\
=\sum_{k=0}^{n-1} \sum_{m=k+1}^{n} \frac{\Gamma(\alpha+m)}{k !} \frac{R^{k}}{z^{m}} \int_{\gamma} \frac{e^{-R \xi} d \sigma(\xi)}{\xi^{m-k}} \\
=\sum_{m=1}^{n} \frac{\Gamma(\alpha+m)}{z^{m}} \sum_{k=0}^{m-1} \frac{R^{k}}{k !} \int_{\gamma} \frac{e^{-R \xi} d \sigma(\xi)}{\xi^{m-k}},
\end{gathered}
$$

and thus,

$$
p_{n}(\alpha)=\Gamma(\alpha+n+1) \sum_{k=0}^{n} \frac{R^{k}}{k !} \int_{\gamma} \frac{e^{-R \xi} d \sigma(\xi)}{\xi^{n+1-k}} .
$$

(iii) Substituting the right-hand side of (119) in the middle sum of (120) yields

$$
\begin{aligned}
\sum_{k=0}^{n-1} & \frac{1}{k !} \frac{R^{k}}{z^{k+1}} I_{2}(R, \alpha, k, n, z) \\
& =\frac{\Gamma(\alpha+n+1)}{z^{n+1}} \sum_{k=0}^{n-1} \frac{R^{k}}{k !} \int_{\gamma} \frac{e^{-R \xi} d \sigma(\xi)}{\xi^{n-k+1}} \frac{1}{\Gamma(\alpha+n+1)} \int_{l_{\theta}} e^{-t} t^{\alpha+n} \frac{\xi z}{\xi z-t} d t,
\end{aligned}
$$

which, with the help of (100), can be rewritten as

$$
\frac{\Gamma(\alpha+n+1)}{z^{n+1}} \sum_{k=0}^{n-1} \frac{R^{k}}{k !} \int_{\gamma} K(-\xi z, \alpha+n) \frac{e^{-R \xi} d \sigma(\xi)}{\xi^{n-k+1}} .
$$

Substituting the right-hand side of (116) in the last sum of (120) yields

$$
\begin{aligned}
& \sum_{k=n}^{\infty} \frac{1}{k !} \frac{R^{k}}{z^{k+1}} I(R, \alpha, k, z) \\
& \quad=\sum_{k=n}^{\infty} \frac{\Gamma(\alpha+k+1)}{k !} \frac{R^{k}}{z^{k+1}} \int_{\gamma} \frac{e^{-R \xi} d \sigma(\xi)}{\xi} \frac{1}{\Gamma(\alpha+k+1)} \int_{l_{\theta}} e^{-t} t^{\alpha+k} \frac{\xi z}{\xi z-t} d t
\end{aligned}
$$

by (100), this can be rewritten as

$$
\sum_{k=n}^{\infty} \frac{\Gamma(\alpha+k+1)}{k !} \frac{R^{k}}{z^{k+1}} \int_{\gamma} K(-\xi z, \alpha+k) \frac{e^{-R \xi} d \sigma(\xi)}{\xi} .
$$

Combining (123) and (124) and using (122), we find integral representations for the remainders

$$
\begin{aligned}
\mathrm{P}_{n, P}(z, \alpha)= & \frac{\Gamma(\alpha+n+1)}{z^{n+1}} \sum_{k=0}^{n-1} \frac{R^{k}}{k !} \int_{\gamma} K(-\xi z, \alpha+n) \frac{e^{-R \xi} d \sigma(\xi)}{\xi^{n-k+1}} \\
& +\sum_{k=n}^{\infty} \frac{\Gamma(\alpha+k+1)}{k !} \frac{R^{k}}{z^{k+1}} \int_{\gamma} K(-\xi z, \alpha+k) \frac{e^{-R \xi} d \sigma(\xi)}{\xi} .
\end{aligned}
$$

Some of the properties of the basic kernel were discussed in Dingle [19] (1973), in $\S 7$ of Chapter 21.

Next we demonstrate further properties of the basic kernel, which we use systematically to prove our main result. 


\section{§6. Properties of the Basic Kernel}

The basic kernel function $K(w, s)$ is central to our development, as indicated in Theorems $4 \mathrm{~A}$ and $4 \mathrm{~B}$, and now we turn to a detailed study of the properties of this function. This includes not only an alternative integral representation for the function together with its analytic continuation to the Riemann surface of $\log w$ in Subsections 6.1 and 6.2, but also the study of its asymptotic properties, in Subsections 6.3 to 6.6. In Subsection 6.7 we apply these results to obtain bounds on the error function $\mathrm{P}_{n}(z, \alpha)$.

Elementary properties of the basic kernel. Clearly, we have

(i) $\quad|K(w, s)| \leq \frac{\Gamma(\operatorname{Re} s+1)}{|\Gamma(s+1)|}, \quad-\frac{\pi}{2} \leq \arg w \leq-\frac{\pi}{2}$,

(ii) $\quad K(w, s) \rightarrow 1, \quad w \rightarrow \infty$,

uniformly for $-\pi+\delta<\arg w<\pi-\delta$.

For any $\delta, 0<\delta \leq \frac{\pi}{2}$, and for $\frac{\pi}{2} \leq|\arg w| \leq \pi-\delta$, estimate (126) deteriorates, taking the form

$$
|K(w, s)| \leq \frac{\Gamma(\operatorname{Re} s+1)}{|\Gamma(s+1)| \sin \delta}, \quad-\pi+\delta<\arg w<\frac{\pi}{2}-\delta .
$$

Remark 5. In what follows, the variable $s$ in (101) is of the form $s=\alpha+n$ with $\alpha$ fixed, $\operatorname{Re}\{\alpha\}>-1$, where $n$ is a nonnegative integer. Therefore, the case where $s>-1$ is of particular interest. It should be noted that in this case we have

$$
\text { (iv) } \quad K(w, s)=K^{*}\left(w^{*}, s\right), \quad-\pi \leq \arg w \leq \pi,
$$

where $*$ denotes complex conjugation. Since $\frac{\Gamma(\operatorname{Re} s+1)}{|\Gamma(s+1)|}=1$, relations (i) and (iii) can be represented in the form

$$
\begin{aligned}
& \text { (i) } \quad|K(w, s)| \leq 1, \quad-\frac{\pi}{2} \leq \arg w \leq-\frac{\pi}{2}, \\
& \text { (iii) }|K(w, s)| \leq \frac{1}{\sin \delta}, \quad-\pi+\delta<\arg w<\frac{\pi}{2}-\delta .
\end{aligned}
$$

\subsection{Identity representation.}

Lemma 2. For $-\frac{\pi}{2} \leq \arg w \leq \frac{\pi}{2}$ and $\operatorname{Re}\{s\}>-1$, we have

$$
\frac{1}{\Gamma(s+1)} \int_{0}^{\infty} e^{-t} t^{s} \frac{w}{t+w} d t \equiv 1-(s+1) \int_{0}^{\infty} \frac{e^{-\lambda w} d \lambda}{(1+\lambda)^{s+2}},
$$

and the integral on the right-hand side of (131) is absolutely convergent $\frac{3}{3}$

We demonstrate two different proofs of this important lemma. The first proof is based on the representation (55) for (44). The second proof is based on the Parceval identity for Fourier transforms.

Proof 1. Rewriting $K(w, s)$ as

$$
K(w, s)=\frac{w^{s+1}}{\Gamma(s+1)} \int_{0}^{\infty} e^{-t w} t^{s} \frac{1}{t+1} d t
$$

and using the parametric representation (44) for $F(t)=\frac{1}{1+t}$, we get

$$
K(w, s)=\frac{w}{\Gamma(s+1)} \int_{0}^{\infty} e^{-t w} F(t, s) d t .
$$

\footnotetext{
${ }^{3}$ Similar relations can be found in Dingle [20] and Olver [26]. However, they have not been explored there.
} 
From (57) it follows that

$$
F(t, s)=\sum_{k=0}^{\infty}(-1)^{k} \frac{\Gamma(s+k+1)}{k !} t^{k},
$$

so that

$$
F(t, s)=\Gamma(s+1) \sum_{k=0}^{\infty}(-1)^{k} \frac{(s+1)_{k}}{k !} t^{k},
$$

where $(s+1)_{k}=(s+1)(s+2) \cdots(s+k)$. Thus,

$$
F(t, s)=\frac{\Gamma(s+1)}{(1+t)^{s+1}},
$$

and substituting (133) in (132) yields

$$
K(w, s)=w \int_{0}^{\infty} \frac{e^{-t w}}{(1+t)^{s+1}} d t
$$

after integration by parts this finally yields (131).

Proof 2. Given $z$ with $\operatorname{Re}\{z\}>0$, consider the following pairs of Fourier transforms:

$$
\frac{1}{\Gamma(s+1)} \int_{0}^{\infty} e^{-t} t^{s} e^{i \lambda t} d t=\frac{1}{(1-i \lambda)^{s+1}}
$$

and

$$
\int_{-\infty}^{\infty} \frac{-i z}{t-i z} e^{i \lambda t} d t= \begin{cases}2 \pi z e^{-\lambda z} & \text { if } \lambda>0 \\ 0 & \text { if } \lambda<0 .\end{cases}
$$

By the Parceval identity,

$$
\frac{1}{\Gamma(s+1)} \int_{0}^{\infty} e^{-t} t^{s} \frac{i z^{*}}{t+i z^{*}} d t=z^{*} \int_{0}^{\infty} \frac{e^{-\lambda z^{*}} d \lambda}{(1-i \lambda)^{s+1}} .
$$

By using Cauchy's theorem, (136) can be rewritten as

$$
\frac{1}{\Gamma(s+1)} \int_{0}^{\infty} e^{-t} t^{s} \frac{i z^{*}}{t+i z^{*}} d t=i z^{*} \int_{0}^{\infty} \frac{e^{-i \lambda z^{*}} d \lambda}{(1+\lambda)^{s+1}} .
$$

Letting $z \rightarrow i w, w>0\left(z^{*} \rightarrow-i w\right)$ in (137) and integrating the last term in (137) by parts, we obtain (131).

\subsection{Analytic continuation and the Stokes phenomenon.}

Lemma 3. (i) Setting $K\left(w e^{i \pi}, s\right)$ and $K\left(w e^{-i \pi}, s\right)$ for the limit values of $K$ on the upper and the lower banks of the negative ray of the w-plane, respectively, for $0 \leq w<+\infty$ we haves

$$
K\left(w e^{-i \pi}, s\right)=1-i(s+1) \int_{0}^{\infty} \frac{e^{i \lambda w}}{(1+i \lambda)^{s+2}} d \lambda
$$

and

$$
K\left(w e^{i \pi}, s\right)=1+i(s+1) \int_{0}^{\infty} \frac{e^{-i \lambda w}}{(1-i \lambda)^{s+2}} d \lambda .
$$

\footnotetext{
${ }^{4}$ The integral representations (138) and (139) form an alternative to the "Smoothing Victorian discontinuity" method by Berry 3 .
} 
(ii) The function $K(w, s)$ admits analytic continuation to the Riemann surface of $\log w$, and we have

$$
K\left(w e^{i \pi}, s\right)=K\left(w e^{-i \pi}, s\right)+2 \pi i \frac{w^{s+1}}{\Gamma(s+1)} e^{-w} .
$$

Identity (140) and the representations (108) and (111) reveal the genuine cause of the occurrence of the Stokes phenomenon.

Proof. (i) Rewriting the right-hand side of (131) as

$$
K(w, s)=w \int_{0}^{\infty} \frac{e^{-\lambda w} d \lambda}{(1+\lambda)^{s+1}}
$$

and using the path of integration given by (47), where $-\pi<\theta<\pi$, and Cauchy's theorem, we have

$$
K\left(w e^{-i \theta}, s\right)=w e^{-i \theta} \int_{l_{\theta}} \frac{e^{-\lambda w e^{-i \theta}} d \lambda}{(1+\lambda)^{s+1}}=w \int_{0}^{\infty} \frac{e^{-\lambda w} d \lambda}{\left(1+\lambda e^{i \theta}\right)^{s+1}} .
$$

Upon setting $\theta=\frac{\pi}{2}$ and $\theta=-\frac{\pi}{2}$, (141) yields

$$
K\left(w e^{-i \frac{\pi}{2}}, s\right)=w \int_{0}^{\infty} \frac{e^{-\lambda w} d \lambda}{(1+i \lambda)^{s+1}}
$$

and

$$
K\left(w e^{i \frac{\pi}{2}}, s\right)=w \int_{0}^{\infty} \frac{e^{-\lambda w} d \lambda}{(1-i \lambda)^{s+1}} .
$$

From (142) and (143) it follows that

$$
K\left(w e^{-i \pi}, s\right)=-i w \int_{0}^{\infty} \frac{e^{i \lambda w} d \lambda}{(1+i \lambda)^{s+1}}
$$

and

$$
K\left(w e^{i \pi}, s\right)=i w \int_{0}^{\infty} \frac{e^{-i \lambda w} d \lambda}{(1-i \lambda)^{s+1}} .
$$

After integration by parts, this yields (138) and (139).

(ii) Representing (145) in the form

$$
K\left(w e^{i \pi}, s\right)=i w \int_{-\infty}^{0} \frac{e^{i \lambda w} d \lambda}{(1+i \lambda)^{s+1}}
$$

(we have replaced $\lambda$ with $-\lambda$ ), and subtracting (144), we get

$$
K\left(w e^{i \pi}, s\right)-K\left(w e^{-i \pi}, s\right)=i w \int_{-\infty}^{+\infty} \frac{e^{i \lambda w} d \lambda}{(1+i \lambda)^{s+1}}
$$

which can be rewritten as

$$
i(s+1) \int_{-\infty}^{+\infty} \frac{e^{i \lambda w} d \lambda}{(1+i \lambda)^{s+2}}=\frac{i(s+1)}{i^{s+2}} \int_{-\infty}^{+\infty} \frac{e^{i \lambda w} d \lambda}{(\lambda-i)^{s+2}} .
$$

If $s$ is an integer, we can evaluate the above integral by applying Cauchy's residue theorem:

$$
\left.2 \pi i \frac{i(s+1)}{i^{s+2}} \frac{1}{\Gamma(s+2)}\left(e^{i \lambda w}\right)^{(s+1)}\right|_{\lambda=i}=2 \pi i \frac{i}{i} \frac{1}{\Gamma(s+1)} w^{s+1} e^{-w} .
$$


For complex $s, \operatorname{Re}\{s\}>-1$, a generalization of the Cauchy residue theorem given by Hankel's contour integral, see [31, (6.1.4)], leads to precisely the same result:

$$
K\left(w e^{i \pi}, s\right)-K\left(w e^{-i \pi}, s\right)=2 \pi i \frac{w^{s+1}}{\Gamma(s+1)} e^{-w} .
$$

For $\operatorname{Re}\{s\}>-1$, set

$$
\mu(s)=\max _{-\pi \leq \arg w \leq \pi}|K(w, s)| .
$$

First, we assume that $s$ is positive and large and study the behavior of $K(w, s)$ with respect to $w$ on the interval $[0, \infty)$. The function $K(w, s)$ is continuous on $[0, \infty)$.

The representation (144) shows that $K(0, s)=0$. With the help of (138), from the Riemann-Lebesgue theorem it follows that $K(w, s) \rightarrow 1$ as $w \rightarrow+\infty$, and this is a particular case of (127). We can expect that $|K(w, s)|$ attains its maximum at a point $w_{0}$ with $0<w_{0}<+\infty$, so that $\mu(s)=\left|K\left(w_{0}, s\right)\right|$. To find the location of $w_{0}$, we use the following heuristic argument. We write the integral found in (138) as

$$
(s+2) \int_{0}^{\infty} e^{\varphi(\lambda)} d \lambda
$$

where

$$
\varphi(\lambda)=(1+i \lambda)-(s+2) \log (1+i \lambda) .
$$

A stationary point $\lambda_{0}$ of $\varphi(\lambda)$ is determined by the equation

$$
\varphi^{\prime}(\lambda)=i w-\frac{i(s+2)}{1+i \lambda}=0, \quad w-(s+2)+i w \lambda=0,
$$

which shows that

$$
w=s+2 \text { and } \lambda=0 .
$$

If $s$ is large, then $w_{0}$ is located in the vicinity of $s+2$.

By using the Taylor expansion

$$
\varphi(\lambda)=\varphi\left(\lambda_{0}\right)+\frac{\left(\lambda-\lambda_{0}\right)}{1 !} \varphi^{\prime}\left(\lambda_{0}\right)+\frac{\left(\lambda-\lambda_{0}\right)^{2}}{2 !} \varphi^{\prime \prime}\left(\lambda_{0}\right)+o\left(\lambda-\lambda_{0}\right)^{2}, \quad \lambda \rightarrow \lambda_{0},
$$

where

$$
\lambda_{0}=0, \quad \varphi(0)=0, \quad \varphi^{\prime}(0)=0, \quad \varphi^{\prime \prime}\left(\lambda_{0}\right)=-\frac{s+2}{2},
$$

we rewrite the integral (148) as

$$
(s+2) \int_{0}^{\infty} e^{(s+2) \frac{\lambda^{2}}{2}+o\left(\lambda-\lambda_{0}\right)^{2}} d \lambda .
$$

It follows that the main contribution to the above integral is given by the expression

$$
(s+2) \int_{0}^{\infty} e^{-(s+2) \frac{\lambda^{2}}{2}} d \lambda=\sqrt{\frac{\pi(s+2)}{2}},
$$

whence

$$
\mu(s) \sim \sqrt{\frac{\pi(s+2)}{2}}, \quad s \rightarrow+\infty .
$$

The above discussion shows that we need to consider two different problems.

1. Given $w$, find an expansion of $K(w, s)$ in terms of an asymptotic series in $\frac{1}{s+2}$.

2. Assuming that $w=s+2+\mu$, where $\mu \in(-s-2, \infty)$ and $\mu$ is fixed, find an asymptotic expansion of $K(s+2+\mu, s)$ in terms of an asymptotic series in $\frac{1}{s+2}$.

Exact solutions of problems 1 and 2 are given by Lemma 4 and Lemma 5 , respectively, which are proved in the next subsection. 
6.3. Asymptotic expansions of $K(w, s)$ and $\mu(s)$ as $s \rightarrow \infty$.

Case where $\operatorname{Re}\{s\}>-1$.

Lemma 4. Let

$$
a_{k}(w)=\frac{\partial^{k} \exp \left(\left(e^{v}-1\right) w+v\right)}{\partial v^{k}}(0, w), \quad k=0,1, \ldots
$$

For any fixed $w,-\pi \leq \arg w \leq \pi, 0 \leq|w|<+\infty$, we have the following asymptotic expansion:

$$
K(w, s) \sim \frac{w}{s+2}+\sum_{k=1}^{\infty} \frac{a_{k}(-w)-a_{k+1}(-w)}{(s+2)^{k+1}}, \quad s \rightarrow \infty .
$$

Proof. (i) After the substitution

$$
v=\log (1+i \lambda)
$$

in the integral found in (138), the function $K\left(w e^{-i \pi}, s\right), 0 \leq w<+\infty$, can be rewritten as

$$
K\left(w e^{-i \pi}, s\right)=1-(s+1) \int_{0}^{\infty} e^{\left(e^{v}-1\right) w+v} \exp (-(s+2) v) d \lambda,
$$

where the integration path is the following curve in the $v$-plane:

$$
v(\lambda)=\frac{1}{2} \log \left(1+\lambda^{2}\right)+i \arctan \lambda, \quad \lambda \in(0,+\infty) .
$$

The first factor in the integrand of (149) can be given by its Taylor series

$$
e^{\left(e^{v}-1\right) w+v}=\sum_{k=0}^{\infty} \frac{a_{k}(w)}{k !} v^{k}
$$

where

$$
a_{k}(w)=\left.\frac{d^{k}}{d v^{k}}\left(e^{\left(e^{v}-1\right) w+v}\right)\right|_{v=0}, \quad k=0,1, \ldots
$$

It is a polynomial of degree $k$. In particular, we have

$$
\begin{aligned}
& a_{0}(w)=1, a_{1}(w)=w+1, \\
& a_{2}(w)=(w+1)^{2}+w, a_{3}(w)=(w+1)^{3}+3(w+1) w+w, \\
& a_{4}(w)=(w+1)^{4}+6(w+1)^{2} w+7 w^{2}+5 w, \ldots
\end{aligned}
$$

The Watson lemma [32, 25, 26] for (149) yields the following asymptotic relation:

$$
K\left(w e^{-i \pi}, s\right) \sim 1-(s+1) \sum_{k=0}^{\infty} \frac{a_{k}(w)}{(s+2)^{k+1}} .
$$

By using (151) and the rearrangement

$$
\begin{aligned}
1-(s+1) \sum_{k=0}^{\infty} \frac{a_{k}(w)}{(s+2)^{k+1}} & =\frac{1}{s+2}-((s+2)-1) \sum_{k=1}^{\infty} \frac{a_{k}(w)}{(s+2)^{k+1}} \\
& =\frac{1}{s+2}-\left(\frac{w+1}{s+2}+\sum_{k=1}^{\infty} \frac{a_{k+1}(w)}{(s+2)^{k+1}}-\sum_{k=1}^{\infty} \frac{a_{k}(w)}{(s+2)^{k+1}}\right),
\end{aligned}
$$

relation (152) can be presented in the form

$$
K\left(w e^{-i \pi}, s\right) \sim \frac{-w}{s+2}-\sum_{k=1}^{\infty} \frac{a_{k+1}(w)-a_{k}(w)}{(s+2)^{k+1}} .
$$


Similarly, we can prove that

$$
K\left(w e^{i \pi}, s\right) \sim \frac{-w}{s+2}-\sum_{k=1}^{\infty} \frac{a_{k+1}(w)-a_{k}(w)}{(s+2)^{k+1}} .
$$

An application of the maximum principle in the complex $w$-plane cut along the negative ray and the fact that the right-hand sides of (153) and (154) coincide show that for any $w$ in the open cut plane we have

$$
K\left(w e^{-i \pi}, s\right) \sim \frac{w}{s+2}-\sum_{k=1}^{\infty} \frac{a_{k+1}(-w)-a_{k}(-w)}{(s+2)^{k+1}} .
$$

Lemma 5. For fixed $\mu$ with $\arg (s+2+\mu)=0$, the following asymptotic expansion holds as $s \rightarrow \infty$ :

$$
\begin{aligned}
K((s & \left.+2+\mu) e^{-i \pi}, s\right) \\
& \sim \frac{5}{3}-i \sqrt{\frac{\pi(s+2)}{2}}-i \sum_{k=1}^{\infty} \frac{\Gamma\left(\frac{k}{2}+1\right) c_{k+2}(\mu)-\Gamma\left(\frac{k}{2}\right) c_{k}(\mu)}{(s+2)^{\frac{k}{2}}},
\end{aligned}
$$

where $c_{k}(\mu), k \in \mathbb{N}$, is a polynomial of degree $k-1$ defined by the expansion

$$
e^{i \lambda(v) \mu} \lambda^{\prime}(v)=\sum_{k=1}^{\infty} c_{k}(\mu) v^{\frac{k}{2}-1}
$$

and $\lambda(v)$ is the function inverse to $v(\lambda)=\log (1+i \lambda)-i \lambda$.

Proof. Below we show that, for any $s$ with $\operatorname{Re}\{s\}>-1$, there exists a finite positive number $w=w(s)$ such that $\left|K\left(w e^{-i \pi}, s\right)\right|=\mu(s)$ and $w(s) \sim s+2$ as $s \rightarrow \infty$. To verify this, we set $w=s+2+\mu$ and rewrite (138) in the form

$$
K\left((s+2+\mu) e^{-i \pi}, s\right)=1-i(s+1) \int_{0}^{\infty} e^{i \lambda \mu} e^{-(s+2)(\log (1+i \lambda)-i \lambda)} d \lambda .
$$

Using the substitution

$$
v(\lambda)=\log (1+i \lambda)-i \lambda
$$

and the convergent expansion

$$
v(\lambda)=\frac{\lambda^{2}}{2}+\sum_{k=3}(-1)^{k+1} \frac{(i \lambda)^{k}}{k}, \quad|\lambda|<1,
$$

we see that the implicit function theorem determines an inverse function $\lambda(v)$ such that

$$
\lambda(v)=\sum_{k=1}^{\infty} a_{k} v^{\frac{k}{2}} .
$$

This series is convergent in the vicinity of $v=0$, and $\lambda(v) \rightarrow+\infty$ as $v \rightarrow-i \infty$. The coefficients $a_{k}$ can be found by substituting (159) into (158). In particular,

$$
a_{1}=\sqrt{2}, \quad a_{2}=\frac{2 i}{3}, \quad a_{3}=\frac{11 \sqrt{2}}{6} .
$$

Relation (157) can be rewritten as

$$
K\left((s+2+\mu) e^{-i \pi}, s\right)=1-i(s+1) \int_{0}^{\infty} e^{i \lambda(v) \mu} \lambda^{\prime}(v) e^{-(s+2) v} d v .
$$

The integration path in (157) is given by

$$
v(\lambda)=\frac{1}{2} \log \left(1+\lambda^{2}\right)-i(\lambda-\arctan \lambda),
$$


and the first factor in the integrand of (161) can be represented in the vicinity of $v=0$ by its Taylor series

$$
e^{i \lambda(v) \mu} \lambda^{\prime}(v)=\sum_{k=1}^{\infty} c_{k}(\mu) v^{\frac{k}{2}-1}
$$

By (159), the coefficients $c_{k}$ can be expressed in terms of $a_{k}$. In particular,

$$
c_{1}(\mu)=\frac{a_{1}}{2}, \quad c_{2}(\mu)=a_{2}+i \frac{a_{1}^{2}}{2} \mu, \quad c_{3}(\mu)=\frac{3 a_{3}}{2}+\frac{3 i a_{1} a_{2}}{2} \mu-\frac{a_{1}^{2}}{2} \mu^{2} .
$$

Using Watson's lemma for the integral in (161), we obtain

$$
K\left((s+2+\mu) e^{-i \pi}, s\right) \sim 1-i(s+1) \sum_{k=1}^{\infty} c_{k} \int_{0}^{\infty} v^{\frac{k}{2}-1} e^{-(s+2) v} d v
$$

as $s \rightarrow \infty$, and evaluating the integrals in (163) yields

$$
K\left((s+2+\mu) e^{-i \pi}, s\right) \sim 1-i(s+1) \sum_{k=1}^{\infty} \frac{\Gamma\left(\frac{k}{2}\right) c_{k}}{(s+2)^{\frac{k}{2}}}, \quad s \rightarrow \infty .
$$

By (160), this becomes

$$
\begin{aligned}
& K\left((s+2+\mu) e^{-i \pi}, s\right) \\
& \quad \sim-i \sqrt{\frac{\pi(s+2)}{2}}+\frac{5}{3}-i \sum_{k=1}^{\infty} \frac{\Gamma\left(\frac{k}{2}+1\right) c_{k+2}-\Gamma\left(\frac{k}{2}\right) c_{k}}{(s+2)^{\frac{k}{2}}} .
\end{aligned}
$$

The next statement follows immediately from (165).

Lemma 6. We have

$$
\mu(s)=\sqrt{\frac{\pi(s+2)}{2}}(1+o(1)), \quad s \rightarrow \infty .
$$

We turn to the case where $\operatorname{Re}\{s\}<-1$; this case differs completely from the above situation of the Stokes phenomenon. When $s$ crosses the line $\operatorname{Re}\{s\}=-1$, the behavior of the kernel $K(w, s)$ as $s \rightarrow \infty$ changes catastrophically and our approach does not work.

Lemma 7. For $\operatorname{Re}\{s\}<-1$ and fixed $w$, the following asymptotic relation holds as $s \rightarrow \infty$ :

$$
K(w, s)=1-\sqrt{\frac{\pi}{w}}(s+1) e^{w+s+2-(s+2) \log \left(-\frac{s+2}{w}\right)}\left(1+O\left(\frac{1}{s}\right)\right) .
$$

Proof. Denoting $\sigma=-(s+2)$, we apply the steepest descent method to the integral

$$
K(w, s)=1+(\sigma+1) \int_{0}^{\infty} e^{-\lambda w+\sigma \log (1+\lambda)} d \lambda,
$$

where $\operatorname{Re}\{\sigma\}>-1$ and $\sigma \rightarrow \infty$. The stationary point $\lambda_{0}$ for the function $\varphi(\lambda)=$ $-\lambda w+\sigma \log (1+\lambda)$ is determined by the equation $\varphi^{\prime}(\lambda)=-w+\frac{\sigma}{1+\lambda}=0$, so that $\lambda_{0}=\frac{\sigma-w}{w}$.

We have

$$
\varphi(\lambda)-\varphi\left(\lambda_{0}\right)=w\left(\lambda-\lambda_{0}\right)^{2} \sum_{k=0}^{\infty} \frac{(-1)^{k+1} w^{k}}{\sigma^{k}(k+1)(k+2)}\left(\lambda-\lambda_{0}\right)^{k}
$$

and

$$
\varphi\left(\lambda_{0}\right)=w-\sigma+\sigma \log \frac{\sigma}{w}
$$


Substituting $\lambda-\lambda_{0}=\sigma \zeta$ in the integral of (167), and using the last two relations, we rewrite formula (167) as

$$
K(w, s)=1+(\sigma+1) \sigma \int_{\frac{1}{w}-\frac{1}{\sigma}}^{\infty} e^{-w \sigma^{2} \zeta^{2} \sum_{k=0}^{\infty} \frac{(-1)^{k} w^{k}}{(k+1)(k+2)} \zeta^{k}} d \lambda .
$$

Applying the implicit function theorem once again, we see that there is a branch $\zeta(v)$ of the inverse function for

$$
v(\zeta)=\zeta^{2} \sum_{k=0}^{\infty} \frac{(-1)^{k+1} w^{k}}{(k+1)(k+2)} \zeta^{k}
$$

that can be represented in the vicinity of $\zeta=0$ by its Taylor series of the form

$$
\zeta(v)=\sum_{k=0}^{\infty} b_{k} \zeta^{\frac{k}{2}}, \quad b_{0}=\sqrt{2} .
$$

Now, we obtain

$$
K(w, s)=1+\sqrt{\frac{\pi}{w}}(\sigma+1) e^{w-\sigma+\sigma \log \frac{\sigma}{w}}\left(1+O\left(\frac{1}{\sigma}\right)\right) .
$$

6.4. Estimates for $K(w, s), 0 \leq|\arg w| \leq \pi$, and for $\mu(s)$. We assume for simplicity that $s$ is real, $s>-1$.

Lemma 8. The function $K(w, s)$ is bounded in the closed $w$-plane cut along the negative ray, and

$$
\begin{gathered}
|K(w, s)| \leq \mu(s), \\
\mu(s)<1+\sqrt{\frac{\pi(s+3)}{2}} ;
\end{gathered}
$$

(ii) the constant $\pi$ in (171) cannot be replaced by a smaller number.

Proof. (i) Formula (138) shows that

$$
\mu(s) \leq 1+(s+1) \int_{0}^{\infty} \frac{1}{\left(1+\lambda^{2}\right)^{\frac{s+2}{2}}} d \lambda,
$$

which can be written as

$$
\mu(s)<1+\frac{s+1}{2} \int_{0}^{\infty} \frac{\lambda^{-\frac{1}{2}}}{(1+\lambda)^{\frac{s+2}{2}}} d \lambda .
$$

Using the well-known relations [31, (6.2.1)-(6.2.2)], we have

$$
\int_{0}^{\infty} \frac{t^{-\frac{1}{2}}}{(1+t)^{\frac{s+2}{2}}} d t=\frac{\Gamma\left(\frac{1}{2}\right) \Gamma\left(\frac{s}{2}+\frac{1}{2}\right)}{\Gamma\left(\frac{s}{2}+1\right)} .
$$

Then, by (172),

$$
\mu(s)<1+\frac{s+1}{2} \sqrt{\pi} \frac{\Gamma\left(\frac{s}{2}+\frac{1}{2}\right)}{\Gamma\left(\frac{s}{2}+1\right)} .
$$

Thus,

$$
\mu(s)<1+\sqrt{\pi} \frac{\Gamma\left(\frac{s+1}{2}+1\right)}{\Gamma\left(\frac{s+1}{2}+\frac{1}{2}\right)} .
$$

Using the inequality

$$
\frac{\Gamma(x)}{\Gamma(y)} \leq \frac{x^{x-\frac{1}{2}} e^{y}}{y^{y-\frac{1}{2}} e^{x}}, \quad x \geq y \geq 1
$$


proved by Kečkić and Vasić (see [33, page 17]), and denoting $\frac{s+1}{2}=r$, we get

$$
\frac{\Gamma(r+1)}{\Gamma\left(r+\frac{1}{2}\right)} \leq \frac{\left(1+\frac{1}{r}\right)^{r}}{\sqrt{e}\left(1+\frac{1}{2 r}\right)^{r}} \sqrt{r+1}<\sqrt{r+1}, \quad r \geq \frac{1}{2} .
$$

Therefore, relation (175) can be written in its final form as

$$
\mu(s)<1+\sqrt{\frac{\pi}{2}(s+3)} .
$$

(ii) This statement follows from a comparison of (176) and (166).

6.5. Estimation of $K(w, s)$ for $\pi \leq|\arg w|<\frac{3 \pi}{2}, s>-1$.

Lemma 9. We have

$$
|K(w, s)| \leq \mu(s)+\frac{\sqrt{2 \pi(s+1)}}{(\cos \theta)^{s+1}}, \quad \pi \leq|\arg w|<\frac{3 \pi}{2}
$$

where $\theta=|\arg w|-\pi$.

Proof. Assume that $\pi \leq \arg w \leq \frac{3}{2} \pi$ or $-\frac{3}{2} \pi \leq \arg w \leq-\pi$. Relation (140) can be represented in the form

$$
K(w, s)=K\left(w e^{-2 i \pi}, s\right)+2 \pi i e^{-i \pi(s+1)} \frac{w^{s+1}}{\Gamma(s+1)} \cdot e^{w} .
$$

Setting $|w|=r$ and $\arg w=\pi+\theta$, we have

$$
|K(w, s)| \leq\left|K\left(r e^{-i(\pi-\theta)}, s\right)\right|+2 \pi \frac{r^{s+1}}{\Gamma(s+1)} \cdot e^{-r \cos \theta} .
$$

Since

$$
\max _{0 \leq r \leq \infty} r^{s+1} e^{-r \cos \theta}=\frac{(s+1)^{s+1}}{(\cos \theta)^{s+1}} e^{-s-1}
$$

and

$$
\frac{1}{\Gamma(s+1)}<\frac{1}{s^{s} e^{-s} \sqrt{2 \pi s}}
$$

from (179) and (170) it follows that

$$
|K(w, s)| \leq \mu(s)+\frac{\sqrt{2 \pi(s+1)}}{(\cos \theta)^{s+1}} .
$$

The fact that an exponentially small term, given by the last term in (140), arises after crossing the ray $\arg z=\frac{\pi}{2}$ leads to the deterioration (177) of the estimate given in (170) and (171). By (171), we have

$$
|K(w, s)| \leq 1+\sqrt{\frac{\pi}{2}(s+3)}+\frac{\sqrt{2 \pi(s+1)}}{(\cos \theta)^{s+1}},
$$

and the last term is the leading term. The case where $-\frac{3}{2} \pi \leq \arg w \leq-\pi$ can be considered similarly. 


\section{$\S 7$. ERROR BOUNDS}

\subsection{Error bounds in the right half-plane.}

\section{Proof of Theorem 4C.}

Remark 6. First, we note that, for fixed $z$, Cauchy's theorem allows us to replace the path of integration $\gamma=\gamma(A, \varepsilon)$ in (111) by a path $\gamma_{1}$ such that some parts of $\gamma_{1}$ belong to the interior of $\mathcal{D}(A, \varepsilon)$. In the parts of the path $\gamma_{1}$ that belong to the open set $\mathcal{D}(A, \varepsilon)$, the measure $d \sigma(\xi)$ should be replaced by $\frac{F(\xi) d \xi}{2 \pi i}$.

We write (see (112))

$$
\gamma(A)=C^{-}(A) \cup\left(l^{+}(A) \cup l^{-}(A)\right)
$$

and consider the integral representation

$$
\begin{aligned}
\mathrm{P}_{n}(z, \alpha) \equiv & \frac{\Gamma(\alpha+n+1)}{z^{n+1}} \sum_{k=0}^{n-1} \frac{R^{k}}{k !} \int_{\gamma(A)} K(-z \xi, \alpha+n) \frac{e^{-R \xi} d \sigma_{1}(\xi)}{\xi^{n-k+1}} \\
& +\sum_{k=n}^{\infty} \frac{\Gamma(\alpha+k+1)}{k !} \frac{R^{k}}{z^{k+1}} \int_{\gamma(A)} K(-z \xi, \alpha+k) \frac{e^{-R \xi} d \sigma_{1}(\xi)}{\xi}
\end{aligned}
$$

where

$$
d \sigma_{1}(\xi)=\left\{\begin{array}{lll}
d \sigma(\xi) & \text { if } \xi \in C^{-}(A), \\
\frac{F(\xi) d \xi}{2 \pi} & \text { if } \xi \in l^{-}(A), \\
\frac{F(\xi) d \xi}{2 \pi i} & \text { if } \quad \xi \in l^{+}(A) .
\end{array}\right.
$$

To estimate $\mathrm{P}_{n}(z, \alpha)$ in the right half-plane $\mathcal{P}(R)$ and to prove that the integrals and sums in (182) are absolutely convergent, we need the following statement.

Lemma 10. Assume that $\xi \in \gamma(A)$. Then for any line $\partial \mathcal{P}\left(R_{1}\right)=\{z \in \mathbb{C}: \operatorname{Re}\{z\}=$ $\left.R_{1}\right\}, R_{1}>R$, and every fixed $z$ on this line we have

$$
-\pi<\arg (-\xi z)<\pi \text {. }
$$

Proof. Relation (19) shows that

$$
\begin{aligned}
& l^{+}(A)=\{t \in \mathbb{C}: t=i A+\rho, 0<\rho<+\infty\}, \\
& l^{-}(A)=\{t \in \mathbb{C}: t=-i A+\rho, 0<\rho<+\infty\} .
\end{aligned}
$$

We have

$$
\begin{aligned}
\frac{\pi}{2} \leq \arg (-\xi)<\pi, & \xi \in l^{-}(A), \\
-\pi<\arg (-\xi) \leq-\frac{\pi}{2}, & \xi \in l^{+}(A),
\end{aligned}
$$

and

$$
\begin{aligned}
0<\arg (-\xi)<\frac{\pi}{2}, & \xi \in C_{A}^{-}, \quad \operatorname{Im}\{\xi\}<0, \\
-\frac{\pi}{2}<\arg (-\xi) \leq 0, & \xi \in C_{A}^{-}, \quad \operatorname{Im}\{\xi\}>0 .
\end{aligned}
$$

We consider two possibilities: (a)

$$
z=R_{1}+i r, r>0 \Longrightarrow 0<\arg z<\frac{\pi}{2}
$$

and (b)

$$
z=R_{1}+i r, r<0 \Longrightarrow-\frac{\pi}{2}<\arg z<0 .
$$


The analysis of the six relations (185)-(192) shows that if $\xi \in C^{-}(A)$ and $z$ satisfies (191) or (192), then relation (184) is fulfilled.

The cases where $\xi \in l^{-}(A)$ or $\xi \in l^{+}(A)$ and $z$ satisfies (191) or (192) require a more thorough analysis.

We consider two cases:

$$
\begin{array}{ll}
{[1]} & \xi \in l^{+}(A) \Longleftrightarrow \xi=i A+\rho, \quad 0<\rho<\infty, \\
{[2]} & \xi \in l^{-}(A) \Longleftrightarrow \xi=-i A+\rho, \quad 0<\rho<\infty .
\end{array}
$$

Then

$$
\begin{aligned}
& \text { [1] } \quad \xi \in l^{+}(A) \Longrightarrow-z \xi=A r-R \rho-i(A R+r \rho), \\
& {[2] \quad \xi \in l^{-}(A) \Longrightarrow-z \xi=-A r-R \rho-i(-A R+r \rho) .}
\end{aligned}
$$

It follows that, for case [1],

$$
\begin{aligned}
& r>0 \Longrightarrow \operatorname{Im}\{(-z \xi)\}<0 \\
& r<0 \Longrightarrow \operatorname{Re}\{(-z \xi)\}<0
\end{aligned}
$$

and, for case [2],

$$
\begin{aligned}
& r>0 \Longrightarrow \operatorname{Re}\{(-z \xi)\}<0, \\
& r<0 \Longrightarrow \operatorname{Im}\{(-z \xi)\}>0 .
\end{aligned}
$$

Relations (193) and (195), and (194) and (196) show that when $\xi$ runs along the boundary of the determinative region, we have

$$
-\pi<\arg (-\xi z)<0 \text { and } \frac{\pi}{2}<\arg (-\xi z)<\pi
$$

and

$$
0<\arg (-\xi z)<\pi \text { and }-\pi<\arg (-\xi z)<-\frac{\pi}{2},
$$

respectively. These two relations complete our proof of (184) 5

Theorem 5B. Assume that $P(z, \alpha)$ is given by (111), and $z \in \mathcal{P}\left(R_{1}\right), R_{1}>R$. Then for every $R_{1}>R$ there exists a positive constant $M_{1}\left(R_{1}\right)$ such that

$$
\left|\mathrm{P}_{n}(z, \alpha)\right| \leq M_{1}\left(R_{1}\right) \mu(\alpha+n) \frac{|\Gamma(\alpha+n+1)|}{A^{n}|z|^{n+1}}, \quad n \in \mathbb{Z}^{+},
$$

where $\mu(\alpha+n)$ is given by (147). Inequality (171) implies that

$$
\mu(\alpha+n)<1+\sqrt{\frac{\pi(|\alpha+n|+3)}{2}} .
$$

Proof. Inequality (184) of Lemma 10 allows us to apply Lemma 8; this yields the estimate

$$
|K(-\xi z, \alpha+k)| \leq \mu(\alpha+k)<1+\sqrt{\frac{\pi(|\alpha|+k+3)}{2}}, \quad k \in \mathbb{Z}^{+},
$$

where $\mu(s)$ is given by (147). For $\xi \in \gamma(A)$ we have $|\xi| \geq A$ and

$$
\frac{1}{|\xi|^{n-k}} \leq \frac{1}{A^{n-k}}, \quad k=0, \ldots, n-1
$$

\footnotetext{
${ }^{5}$ It can be predicted with certainty that there should exist a simpler and more transparent proof of this lemma.
} 
Using (198) and (199) and taking into account the condition

$$
\int_{\gamma} \frac{e^{-R \xi}\left|d \sigma_{1}(\xi)\right|}{|\xi|}<M
$$

which follows from (103), we can employ (182) to deduce the following inequality:

$$
\begin{aligned}
\left|\mathrm{P}_{n}(z, \alpha)\right| \leq & M \mu(\alpha+n) \frac{|\Gamma(\alpha+n+1)|}{A^{n}|z|^{n+1}} \sum_{k=0}^{n-1} \frac{(R A)^{k}}{k !} \\
& +M|\Gamma(\alpha+1)| \sum_{k=n}^{\infty} \mu(\alpha+k) \frac{(\alpha+1)_{k}}{k !} \frac{R^{k}}{|z|^{k+1}} .
\end{aligned}
$$

Here the second term is the $n$th remainder of the convergent power series for $R<|z|<\infty$. It is negligibly small in comparison with the leading term. Thus, replacing the constant $M$ by a greater constant $M_{1}\left(R_{1}\right)$ for $R<R_{1}<|z|<\infty$, we finally have

$$
\left|\mathrm{P}_{n}(z, \alpha)\right| \leq M_{1}\left(R_{1}\right) \mu(\alpha+n) \frac{|\Gamma(\alpha+n+1)|}{A^{n}|z|^{n+1}}, \quad n \in \mathbb{Z}^{+} .
$$

The last estimate together with the result of the subsection "Formal derivation" completes the proof of Theorem $4 \mathrm{C}$.

Assume that $R=0$. Then we have a stronger result than that given by Theorem 5B.

Theorem 5A. Assume that $P(z, \alpha)$ is given by (182), and let $\operatorname{Re} z \geq 0, z \neq 0$. Then

$$
\left|\mathrm{P}_{n}(z, \alpha)\right| \leq M \mu(\alpha+n) \frac{|\Gamma(\alpha+n+1)|}{A^{n}|z|^{n+1}}, \quad n \in \mathbb{Z}^{+},
$$

where $M$ is given by (103) and $\mu(\alpha+n)$ is given by (147).

Proof. The representation (182) can be rewritten as

$$
\mathrm{P}_{n}(z, \alpha) \equiv \frac{\Gamma(\alpha+n+1)}{z^{n+1}} \int_{\gamma(A)} \frac{K(-\xi z, \alpha+n) d \sigma_{1}(\xi)}{\xi^{n+1}}, \quad n \in \mathbb{N},
$$

where $\sigma_{1}(\xi)$ is given by (183). Then the estimates $|K(-\xi z, \alpha+n)| \leq \mu(\alpha+n)$ and (103) immediately yield estimate (201) for $\operatorname{Re}\{z\} \geq R$, and we recall that

$$
\mu(\alpha+n)<1+\sqrt{\frac{\pi(|\alpha+n|+3)}{2}} .
$$

Remark 7. Estimate (201) is sharper than that given by (200). Moreover, it is valid in the closed right half-plane $\mathcal{P}(R)$. We suspect that Theorem $5 \mathrm{~B}$ can be improved, and estimate (200) can be replaced by (201). A possible way to do this is to provide the space $W(R, A)$ with a topology, and to show that $W(0, A)$ is a dense set in $W(R, A)$ given by (22), $\varepsilon=0$.

Remark 8 . The Poincaré asymptotic theory usually deals with approximations in regions of the form

$$
\left\{z \in \mathbb{C}:-\frac{\pi}{2}+\delta \leq \arg z \leq \frac{\pi}{2}-\delta, R<|z|<\infty, 0<\delta \leq \frac{\pi}{2}\right\}
$$

and cannot clarify what happens along the line parallel to the imaginary axis. A typical example is the estimates for the remainders in the Watson lemma. In contrast to the Poincaré theory, our approach allows us to do that. 
7.2. Error bounds in regions $\mathcal{P}(R, \theta), 0 \leq \theta \leq \varepsilon$.

Proof of Theorem 4B. Consider the integral representation

$$
\begin{aligned}
\mathrm{P}_{n}(z, \alpha) \equiv & \frac{\Gamma(\alpha+n+1)}{z^{n+1}} \sum_{k=0}^{n-1} \frac{R^{k}}{k !} \int_{\gamma(A, \varepsilon)} \widetilde{K}(-z \xi, \alpha+n) \frac{e^{-R \xi} d \sigma(\xi)}{\xi^{n-k+1}} \\
& +\sum_{k=n}^{\infty} \frac{\Gamma(\alpha+k+1)}{k !} \frac{R^{k}}{z^{k+1}} \int_{\gamma(A, \varepsilon)} \widetilde{K}(-z \xi, \alpha+k) \frac{e^{-R \xi} d \sigma(\xi)}{\xi},
\end{aligned}
$$

where $\gamma(A, \varepsilon)$ is given by (19).

To estimate $\mathrm{P}_{n}(z, \alpha)$ in the region $\mathcal{P}(R, \varepsilon), 0 \leq \varepsilon<\frac{\pi}{2}$, we require the next statement, which follows from Lemma 10 by an elementary argument.

Lemma 11. Assume that $\xi \in \gamma(A)$. Then for $z \in \mathcal{P}(R, \theta), 0 \leq \theta \leq \varepsilon$, we have

$$
-\pi-\theta<\arg (-\xi z)<\pi+\theta .
$$

This inequality and Lemma 9 yield the estimate

$$
|\widetilde{K}(-z \xi, \alpha+k)| \leq \mu(|\alpha|+k)+\frac{\sqrt{2 \pi(|\alpha|+k+1)}}{(\cos \theta)^{\operatorname{Re} \alpha+k+1}} .
$$

Now we can repeat the proof of Theorem 5, replacing (198) by (204), to show that for every $z \in \mathcal{P}(R, \varepsilon)$ all the integrals and sums in (111) are absolutely convergent. We also have uniform error bounds in every region $\mathcal{P}\left(R_{1}, \theta\right)$, where $0 \leq \theta \leq \varepsilon$ and $R<R_{1}<|z|<\infty$. This proves the following result.

Theorem 6B. Assume that $P(z, \alpha)$ is given by (111), and let $z \in \mathcal{P}\left(R_{1}, \varepsilon\right)$, where $R<R_{1}$. Then there exists a positive constant $M_{1}\left(R_{1}\right)$ such that for $n \in \mathbb{Z}^{+}$we have

$$
\left|\mathrm{P}_{n}(z, \alpha)\right| \leq M_{1}\left(R_{1}\right)\left(1+\sqrt{\frac{\pi(\alpha+n+3)}{2}}+\frac{\sqrt{2 \pi(\alpha+n+1)}}{(\cos \theta)^{\alpha+n+1}}\right) \frac{\Gamma(\alpha+n+1)}{A^{n}|z|^{n+1}} .
$$

Theorem $6 \mathrm{~B}$ finalizes the proof of Theorem 4B.

However, for the case where $R=0$ in Theorem 4A, we have a much better result. Compare with Remark 7.

Theorem 6A. Assume that $\alpha>-1$ and that $\mathrm{P}_{n}(z, \alpha), n=0,1, \ldots$, is given by

$$
\mathrm{P}_{n}(z, \alpha)=\frac{\Gamma(\alpha+n+1)}{z^{n+1}} \int_{\gamma(A, \varepsilon)} \frac{K_{\varepsilon}(-\xi z, \alpha+n) d \sigma(\xi)}{\xi^{n+1}},
$$

where

$$
\int_{\gamma(A, \varepsilon)}\left|\frac{d \sigma(\xi)}{\xi}\right| \leq M
$$

Then

(i) for $\operatorname{Re} z \geq 0$ we have

$$
\left|\mathrm{P}_{n}(z, \alpha)\right| \leq M \mu(\alpha+n) \frac{\Gamma(\alpha+n+1)}{A^{n}|z|^{n+1}}, \quad n \in \mathbb{Z}^{+},
$$

where $\gamma(s)$ is given by (147);

(ii) for $-\frac{\pi}{2}-\varepsilon<\arg z<\frac{\pi}{2}+\varepsilon$ we have

$$
\begin{array}{r}
\left|\mathrm{P}_{n}(z, \alpha)\right| \leq M\left(1+\sqrt{\frac{\pi(\alpha+n+3)}{2}}+\frac{\sqrt{2 \pi(\alpha+n+1)}}{(\cos \theta)^{\alpha+n+1}}\right) \frac{\Gamma(\alpha+n+1)}{A^{n}|z|^{n+1}}, \\
n \in \mathbb{Z}^{+},
\end{array}
$$

where $\theta=|\arg z|-\frac{\pi}{2}$. 
Proof. Using Lemma 11 and estimates (204) and (207), we obtain inequality (209) immediately from (206).

To prove (201), we assume that $-\frac{\pi}{2}<\arg z<\frac{\pi}{2}$. Then, using the Cauchy theorem and Remark 6, we can deform the contour $\gamma(A, \varepsilon)$ to $\gamma(A)$, returning to the representation

$$
\mathrm{P}_{n}(z, \alpha)=\frac{\Gamma(\alpha+n+1)}{z^{n+1}} \int_{\gamma(A)} \frac{\widetilde{K}(-\xi z, \alpha+n) d \sigma_{1}(\xi)}{\xi^{n+1}},
$$

where $\sigma_{1}(\xi)$ is given by (183) and $\widetilde{K}(-\xi z, \alpha+n)=K(-\xi z, \alpha+n)$.

A comparison of estimates (208) and (209) shows that, upon crossing either the positive or the negative part of the imaginary line in the $z$-plane, the error bound deteriorates, and the greater is the deviation of $\arg z$ from $\frac{\pi}{2}$ or $-\frac{\pi}{2}$, the greater is the deterioration in the error bound.

\section{$\S 8$. STOKES' PHENOMENON}

In what follows we clarify the cause of the deterioration that we saw above in (203) and (204).

To simplify our arguments, we assume that $R=0$. All our calculations can be adapted then for the general case where $R>0$, with the only difference that this would lead to rather cumbersome expressions.

Let the contour $\gamma(A, \varepsilon)$ of the $t$-plane be given by (19), so that

$$
\gamma(A, \varepsilon)=l^{+}(A, \varepsilon) \cup C^{-}(A) \cup l^{-}(A, \varepsilon)
$$

and

$$
F(t)=\int_{\gamma(A, \varepsilon)} \frac{d \sigma(\xi)}{\xi-t}, \quad t \in \mathcal{D}(A, \varepsilon),
$$

where $\mathcal{D}(A, \varepsilon)$ and $\gamma=\gamma(A, \varepsilon)$ are given by (18) and (19), respectively, and $\sigma$ is a measure supported on $\gamma$. We assume also that

$$
\int_{\gamma(A, \varepsilon)} \frac{|d \sigma(\xi)|}{|\xi|}<M
$$

for some positive $M$.

Set

$$
\begin{aligned}
P(z, \alpha) & =z^{\alpha} \int_{0}^{+\infty} e^{-t z} t^{\alpha} F(t) d t, \\
p_{n}(\alpha) & =\frac{\Gamma(\alpha+n+1)}{n !} F^{(n)}(0),
\end{aligned}
$$

and

$$
\mathrm{P}_{n}(z)=P(z)-\sum_{k=0}^{n-1} \frac{p_{k}}{z^{k+1}}, \quad n \in \mathbb{Z}^{+} .
$$

Theorems $4 \mathrm{~A}$ and $6 \mathrm{~A}$ imply that $\mathrm{P}_{n}(z)$ admits analytic continuation to the region

$$
\mathcal{P}(\varepsilon)=\left\{z \in \mathbb{C}:-\frac{\pi}{2}-\varepsilon<\arg z<\frac{\pi}{2}+\varepsilon\right\} .
$$

For $z \in \mathcal{P}(\varepsilon)$ we have

$$
\mathrm{P}_{n}(z, \alpha)=\frac{\Gamma(\alpha+n+1)}{z^{n+1}} \int_{\gamma(A, \varepsilon)} \frac{K_{\varepsilon}(-\xi z, \alpha+n) d \sigma(\xi)}{\xi^{n+1}},
$$

where $\widetilde{K}(-\xi z, \alpha+n)$, given by (105), is bounded on the path of integration. 
We represent the sector $\mathcal{P}(\varepsilon)$ of the $z$-plane as the union

$$
\mathcal{P}(\varepsilon)=\mathcal{P}^{+}(\varepsilon) \cup \mathcal{P} \cup \mathcal{P}^{-}(\varepsilon),
$$

where

$$
\begin{aligned}
& \mathcal{P}^{+}(\varepsilon)=\left\{z \in \mathbb{C}: \frac{\pi}{2}<\arg z<\frac{\pi}{2}+\varepsilon\right\}, \\
& \mathcal{P}^{-}(\varepsilon)=\left\{z \in \mathbb{C}:-\frac{\pi}{2}-\varepsilon<\arg z<-\frac{\pi}{2} \varepsilon\right\},
\end{aligned}
$$

and $\mathcal{P}$ is the right half-plane.

Set

$$
E^{+}(z, \alpha)=2 \pi i \cdot z^{\alpha} \int_{l^{-}(A, \varepsilon)} e^{-z t} t^{\alpha} d \sigma(t), \quad z \in \mathcal{P}^{+}(\varepsilon)
$$

and

$$
E^{-}(z, \alpha)=2 \pi i \cdot z^{\alpha} \int_{l^{+}(A, \varepsilon)} e^{-z t} t^{\alpha} d \sigma(t), \quad z \in \mathcal{P}^{-}(\varepsilon),
$$

where the rays $l^{+}(A, \varepsilon)$ and $l^{-}(A, \varepsilon)$ are given by (210).

Theorem 7A. Assume that all conditions of Theorem 6A are satisfied. Assume that $E^{+}(z, \alpha)$ and $E^{-}(z, \alpha)$ are given by (221) and (222), respectively. Then we have the following representations for $\mathrm{P}_{n}(z, \alpha), n \in \mathbb{Z}^{+}$, in the regions $\mathcal{P}, \mathcal{P}^{+}(\varepsilon)$, and $\mathcal{P}^{-}(\varepsilon)$ :

- (i) for $z \in \mathcal{P}$,

$$
\mathrm{P}_{n}(z, \alpha) \equiv \frac{\Gamma(\alpha+n+1)}{z^{n+1}} \int_{\gamma(A, \varepsilon)} \frac{K(-\xi z, \alpha+n) d \sigma(\xi)}{\xi^{1+n}} ;
$$

- (ii) for $z \in \mathcal{P}^{+}(\varepsilon)$,

$$
\mathrm{P}_{n}(z, \alpha) \equiv E^{+}(z, \alpha)+\frac{\Gamma(\alpha+n+1)}{z^{n+1}} \int_{\gamma(A, \varepsilon)} \frac{K(-\xi z, \alpha+n) d \sigma(\xi)}{\xi^{1+n}} ;
$$

- (iii) for $z \in \mathcal{P}^{-}(\varepsilon)$, the function $P(z, \alpha)$ can be represented in the form

$$
\mathrm{P}_{n}(z, \alpha) \equiv E^{-}(z, \alpha)+\frac{\Gamma(\alpha+n+1)}{z^{n+1}} \int_{\gamma(A, \varepsilon)} \frac{K(-\xi z, \alpha+n) d \sigma(\xi)}{\xi^{1+n}} ;
$$

and in all three integral representations for every $\xi \in \gamma(A, \varepsilon)$ we have

$$
-\pi<\arg (-\xi z)<\pi .
$$

Proof. Part (i) was, in fact, proved in Theorem 6A; see (202). To prove (ii) and (iii), we rewrite the integral representation for $\mathrm{P}_{n}(z, \alpha)$ given by (206) in the form

$$
\begin{aligned}
\mathrm{P}_{n}(z, \alpha) \equiv & \frac{\Gamma(\alpha+n+1)}{z^{n+1}} \int_{l^{-}(A, \varepsilon)} \frac{K\left(\xi z e^{i \pi}, \alpha+n\right) d \sigma(\xi)}{\xi^{1+n}} \\
& +\frac{\Gamma(\alpha+n+1)}{z^{n+1}} \int_{l^{+}(A, \varepsilon)} \frac{K\left(\xi z e^{-i \pi}, \alpha+n\right) d \sigma(\xi)}{\xi^{1+n}} \\
& +\frac{\Gamma(\alpha+n+1)}{z^{n+1}} \int_{C^{-}(A)} \frac{K(-\xi z, \alpha+n) d \sigma(\xi)}{\xi^{1+n}}, \quad n \in \mathbb{Z}^{+} .
\end{aligned}
$$

Then we apply the relation

$$
K\left(w e^{i \pi}, s\right)=K\left(w e^{-i \pi}, s\right)+2 \pi i \frac{w^{s+1}}{\Gamma(s+1)} e^{-w}
$$


of Lemma 3 to the first integral in (227). Suppose for definiteness that $z \in \mathcal{P}^{+}(\varepsilon)$. Then, using the formula

$$
K\left(\xi z e^{i \pi}, \alpha+n\right)=2 \pi i \frac{(\xi z)^{\alpha+n+1}}{\Gamma(\alpha+n+1)} e^{-\xi z}+K\left(\xi z e^{-i \pi}, \alpha+n\right),
$$

we rewrite the first integral of (227) as

$$
\begin{aligned}
& \frac{\Gamma(\alpha+n+1)}{z^{n+1}} \int_{l^{-}(A, \varepsilon)} 2 \pi i \frac{(\xi z)^{\alpha+n+1}}{\Gamma(\alpha+n+1)} e^{-\xi z} \frac{d \sigma(\xi)}{\xi^{1+n}} \\
& +\frac{\Gamma(\alpha+n+1)}{z^{n+1}} \int_{l^{-}(A, \varepsilon)} K\left(\xi z e^{-i \pi}, \alpha+n\right) \frac{F(\xi) d \xi}{\xi^{1+n}} .
\end{aligned}
$$

An obvious simplification of the last expression yields

$$
2 \pi i \cdot z^{\alpha} \int_{l^{-}(A, \varepsilon)} e^{-\xi z} \xi^{\alpha} d \sigma(\xi)+\frac{\Gamma(\alpha+n+1)}{z^{n+1}} \int_{l^{-}(A, \varepsilon)} K\left(\xi z e^{-i \pi}, \alpha+n\right) \frac{d \sigma(\xi)}{\xi^{1+n}},
$$

which by (221) is equal to

$$
E^{+}(z, \alpha)+\frac{\Gamma(\alpha+n+1)}{z^{n+1}} \int_{l^{-}(A, \varepsilon)} K\left(\xi z e^{-i \pi}, \alpha+n\right) \frac{F(\xi) d \xi}{\xi^{1+n}} .
$$

Substituting the last expression for the first integral in (227) and combining all the integrals yields (224). It remains to observe that for $\xi \in \gamma(A, \varepsilon)$ and for

$$
z \in \mathcal{P}^{+}(\varepsilon)=\left\{z \in \mathbb{C}: \frac{\pi}{2}<\arg z<\frac{\pi}{2}+\varepsilon\right\}
$$

we have

$$
-\pi<\arg \left(\xi z e^{-i \pi}\right)<\pi
$$

which completes the proof of (ii). Statement (iii) can be proved similarly.

Next we show that the functions $E^{+}(z, \alpha)$ and $E^{-}(z, \alpha)$ decay exponentially as $z \rightarrow \infty$ in the regions $\mathcal{P}^{+}(\varepsilon)$ and $\mathcal{P}^{-}(\varepsilon)$, respectively.

Theorem 8A. Assume that all conditions of Theorem 6A are satisfied. Setting $z=$ $r e^{i\left(\frac{\pi}{2}+\theta\right)}$ for $z \in \mathcal{P}^{+}(\varepsilon)$ and $z=r e^{-i\left(\frac{\pi}{2}+\theta\right)}$ for $z \in \mathcal{P}^{-}(\varepsilon)$, where $0 \leq \theta \leq \varepsilon_{1}<\varepsilon$ and $r>0$, we have

$$
E^{+}(z, \alpha)=z^{\alpha} \int_{l^{-}\left(A, \varepsilon_{1}\right)} e^{-z t} t^{\alpha} F(t) d t, \quad z \in \mathcal{P}^{+}\left(\varepsilon_{1}\right)
$$

and

$$
E^{-}(z, \alpha)=z^{\alpha} \int_{l^{+}\left(A, \varepsilon_{1}\right)} e^{-z t} t^{\alpha} F(t) d t, \quad z \in \mathcal{P}^{-}\left(\varepsilon_{1}\right)
$$

Moreover,

$$
E^{+}(z, \alpha)=O\left(r^{\operatorname{Re}\{\alpha\}} e^{-A r \cos \theta}\right), \quad r \rightarrow+\infty, \frac{\pi}{2}<\arg z<\frac{\pi}{2}+\varepsilon_{1},
$$

and

$$
E^{-}(z, \alpha)=O\left(r^{\operatorname{Re}\{\alpha\}} e^{-A r \cos \theta}\right), \quad r \rightarrow+\infty,-\frac{\pi}{2}-\varepsilon_{1}<\arg z<-\frac{\pi}{2},
$$

and these estimates are uniform in $\arg z$ in the sectors $\mathcal{P}^{+}\left(\varepsilon_{1}\right)$ and $\mathcal{P}^{-}\left(\varepsilon_{1}\right)$. 
Proof. Assuming that $z \in \mathcal{P}^{+}\left(\varepsilon_{1}\right)$ or $z \in \mathcal{P}^{-}\left(\varepsilon_{1}\right), 0<\varepsilon_{1}<\varepsilon$, and using Cauchy's theorem, we can rewrite (221) and (222) in the form

$$
E^{+}(z, \alpha)=z^{\alpha} \int_{l^{-}\left(A, \varepsilon_{1}\right)} e^{-\xi z} \xi^{\alpha} F(\xi) d \xi
$$

or

$$
E^{-}(z, \alpha)=z^{\alpha} \int_{l^{+}\left(A, \varepsilon_{1}\right)} e^{-\xi z} \xi^{\alpha} F(\xi) d \xi
$$

respectively.

To prove (230), we note that, by the change $t \rightarrow-i A+\xi$, from (228) it follows that

$$
E^{+}(z, \alpha)=z^{\alpha} e^{i A z} \int_{l_{-\varepsilon}} e^{-z \xi}(-i A+\xi)^{\alpha} F(-i A+\xi) d \xi
$$

where the path $l_{-\varepsilon}$ is given by (43) with $\theta=-\varepsilon$, and the last integral yields estimate (230). A similar estimate can be obtained for $E^{-}(z, \alpha)$, by using the integral representation

$$
E^{-}(z, \alpha)=z^{\alpha} e^{-i A z} \int_{l_{\varepsilon}} e^{-z \xi}(i A+\xi)^{\alpha} F(i A+\xi) d \xi .
$$

A comparison of (223) with (224) and (225) shows that upon and after crossing the positive and the negative part of the imaginary line, the new terms (221) and (222) arise, respectively. These terms are exponentially small with exponent determined by $A$. Nevertheless, these terms should be taken into account for more accurate calculations of the values of $P(z, \alpha)$ in the left half-plane. Evaluating the power series expansions in $\frac{1}{z}$ for the two integrals in (232) and (233), we obtain the so-called asymptotics beyond all orders.

Remark 9. It should be noted that if the function $P(z, \alpha)$ is generated by a solution of a linear ODE with an irregular singularity at $\infty$, then the form of the three integrals (44), (228), and (229) allows us to expect that the functions $E^{+}(z, \alpha)$ and $E^{-}(z, \alpha)$ can be expressed in terms of other solutions of the same ODE.

The arising of such terms upon crossing certain lines in the complex plane is usually recognized as a manifestation of the Stokes phenomenon and these lines are called the Stokes lines.

The error bounds (208) given by Theorem $5 \mathrm{~A}$ in the right half-plane are very sharp. We show that the error bounds (209) in the region $\mathcal{P}^{+}(\varepsilon) \cup \mathcal{P}^{-}(\varepsilon)$ can be improved.

\subsection{Improved error bounds in the regions $\mathcal{P}^{-}(R, \varepsilon)$.}

Theorem 9A. Assume that all conditions of Theorem 6A are satisfied. Then:

(i) for $\operatorname{Re} z \geq 0$ we have

$$
\left|\mathrm{P}_{n}(z, \alpha)\right| \leq M \mu(\alpha+n) \frac{|\Gamma(\alpha+n+1)|}{A^{n}|z|^{n+1}}, \quad n \in \mathbb{Z}^{+} ;
$$

(ii) for $z \in \mathcal{P}^{+}(\varepsilon) \cup \mathcal{P}^{-}(\varepsilon)$ we have

$$
\left|\mathrm{P}_{n}(z, \alpha)\right| \leq M \frac{|\Gamma(\alpha+n+1)|}{A^{n}|z|^{n+1}}\left(\mu(\alpha+n)+\frac{\sqrt{2 \pi(\alpha+n+1)}}{(\cos \theta)^{\alpha+n+1}}\right), \quad n \in \mathbb{Z}^{+},
$$

and $\theta=|\arg z|-\frac{\pi}{2}$

(iii) for $z \in \mathcal{P}^{+}(\varepsilon)$ we have

$$
\left|\mathrm{P}_{n}(z, \alpha)-E^{+}(z, \alpha)\right| \leq M \mu(\alpha+n) \frac{|\Gamma(\alpha+n+1)|}{A^{n}|z|^{n+1}}, \quad n \in \mathbb{Z}^{+}
$$


(iv) for $z \in \mathcal{P}^{-}(\varepsilon)$ we have

$$
\left|\mathrm{P}_{n}(z, \alpha)-E^{-}(z, \alpha)\right| \leq M \mu(\alpha+n) \frac{|\Gamma(\alpha+n+1)|}{A^{n}|z|^{n+1}}, \quad n \in \mathbb{Z}^{+} .
$$

Proof. Parts (i) and (ii) were already proved in Theorem 5A and Theorem 6A, respectively. From (224) it follows that

$$
\mathrm{P}_{n}(z, \alpha)-E^{+}(z, \alpha)=\frac{\Gamma(\alpha+n+1)}{z^{n+1}} \int_{\gamma(A, \varepsilon)} \frac{K(-\xi z, \alpha+n) d \sigma(\xi)}{\xi^{1+n}},
$$

which, together with (226) and (103), implies (236). The proof of (237) is similar.

Thus, Part (ii) of Theorem 9 shows precisely how the exponentially small terms $E^{+}(z, \alpha)$ and $E^{-}(z, \alpha)$, given by (232) and (233), respectively, affect the previously good estimate given by part (ii): the second term in brackets in (234) is clearly the leading term; compare it with $\mu(\alpha+n)$ satisfying (198). Moreover, parts (iii) and (iv) show that adding these terms to the finite sum $\sum_{k=0}^{n-1} \frac{p_{k}(\alpha)}{z^{k+1}}$ retains the previous accuracy for the error.

A very important consequence of this result is a strong domination of the terms $E^{+}(z, \alpha)$ and $E^{-}(z, \alpha)$ over the remainder $\mathrm{P}_{n}(z, \alpha)$ in the left half-plane.

Derivation of Theorems $7 \mathrm{~A}-9 \mathrm{~A}$ (case where $R=0$ ) and Theorems $4 \mathrm{~B}-6 \mathrm{~B}$ allow us to provide similar derivations for the case $R>0$ and to state and prove the more general Theorems 7B-9B.

Example 3. Stokes' phenomenon for the Stirling expansion for $\log \Gamma(z)$. (For an earlier discussion, see 34, 35, 36, 4, 20, 26, 6 and 37,6.)

The Stirling function $P(z)$ given by (9) can be represented as

$$
P(z)=\int_{0}^{\infty} e^{-t z} F(t) d t
$$

where

$$
F(t)=2 \sum_{k=1}^{\infty} \frac{1}{t^{2}+4 \pi^{2} k^{2}}
$$

The function (239) can be rewritten in the form

$$
F(t)=\sum_{k=-\infty}^{k=+\infty} \frac{1}{2 \pi i k(t-2 \pi i k)}=\int_{-i \infty}^{i \infty} \frac{d \sigma(\xi)}{\xi(t-\xi)}=\int_{i \infty}^{-i \infty} \frac{d \sigma(\xi)}{\xi(\xi-t)},
$$

where $\sigma(\xi)$ is a step function with jump 1 at every point $\pm 2 \pi i k, k \in \mathbb{N}$.

It follows that $F(t) \in B\left(0,2 \pi, \frac{\pi}{2}\right)$. After applying (108), the remainders $\mathrm{P}_{n}(z)$ of the function $P(z)$ can be represented as

$$
\mathrm{P}_{n}(z) \equiv \frac{\Gamma(n+1)}{z^{n+1}} \int_{\gamma} \frac{K(-\xi z, n) d \sigma(\xi)}{\xi^{n+2}}
$$

where $\gamma=\gamma\left(A, \frac{\pi}{2}\right)$, with any $A, 0<A<2 \pi$, and we have

$$
P(z) \equiv \frac{1}{z} \int_{\gamma\left(A, \frac{\pi}{2}\right)} \frac{K(-\xi z, 0) d \sigma(\xi)}{\xi^{2}} .
$$

\footnotetext{
${ }^{6}$ The argument of the last paper can be applied to $\log \boldsymbol{\Gamma}(\mathbf{z})$.
} 
Applying formulas (224) and (225), we obtain

$$
\begin{aligned}
& E^{+}(z) \equiv \frac{1}{z} \int_{-i \infty}^{-i A} \frac{2 \pi i \xi z e^{-\xi z} d \sigma(\xi)}{\xi^{2}} \\
& E^{+}(z) \equiv 2 \pi i \int_{-i A}^{-i \infty} \frac{e^{-\xi z} d \sigma(\xi)}{\xi}=2 \pi i \sum_{k=1}^{\infty} \frac{e^{2 \pi i k z}}{-2 \pi i k}=-\log \left(1-e^{2 \pi i z}\right), \\
& E^{-}(z) \equiv 2 \pi i \int_{i \infty}^{i A} \frac{e^{-\xi z} d \sigma(\xi)}{\xi}=-\log \left(1-e^{-2 \pi i z}\right),
\end{aligned}
$$

so that

$$
E^{ \pm}(z)=-\log \left(1-e^{ \pm 2 \pi i z}\right)
$$

A formula close to (241) was discovered by Hermite [34] (1882), who used it to prove the reflection formula for the gamma function. Formula (241) was proved in Lindelöf [35] (1905). Both were unaware of the work of Stokes and derived for the Stirling case a formula which anticipated the Sokhotsky-Plemelj formula.

A further investigation of the Stokes phenomenon for this case, based on ideas of superasymptotics and hyperasymptotics, appeared in Berry [4 (1991a). See also [6].

Applying Theorem A to (240) gives the error bounds for the Stirling expansion shown in Subsection 1.2.

8.2. Duality theorem. Now we have all the techniques to prove Theorem 1, and the proof is very short.

Let the spaces $\mathbf{B}(R, A, \varepsilon)$ and $\mathbf{W}(R, A, \varepsilon)$ be given by (37) and (38), respectively.

Assume that $F(t) \in \mathbf{B}(R, A, \varepsilon)$. It follows that for every $A^{\prime}$ with $0<A^{\prime}<A, F(t) \in$ $B\left(R, A^{\prime}, \varepsilon\right)$, and we can apply Theorem $4 \mathrm{~B}$ to introduce the corresponding function $P(z, \alpha)$ and to check that $P(z, \alpha)$ satisfies all conditions of Theorem $6 \mathrm{~A}$ in the region $\mathcal{P}\left(R^{\prime}, \varepsilon^{\prime}\right)$ for every $R<R^{\prime}<\infty$ and $0<\varepsilon^{\prime}<\varepsilon$. Therefore, $P(z, \alpha) \in \mathbf{W}(R, A, \varepsilon)$, and this shows that

$$
\mathbf{B}(R, A, \varepsilon) \subset \mathcal{L}_{\alpha}^{-1} \mathbf{W}(R, A, \varepsilon) .
$$

Now, we assume that $P(z) \in \mathbf{W}(R, A, \varepsilon)$ for $z \in \mathcal{P}(R, \varepsilon)$. We represent $\mathcal{P}(R, \varepsilon)$ as

$$
\mathcal{P}(R, \varepsilon)=\bigcup_{-\varepsilon<\theta<\varepsilon} e^{i \theta} \mathcal{P}(R)
$$

and apply Nevanlinna's theorem for every half-plane $e^{i \theta} \mathcal{P}(R)$; this shows that $P(z)=$ $\mathcal{L} G(t)$, where $G(t) \in B\left(R^{\prime}, A^{\prime}, \varepsilon^{\prime}\right)$ for every $0<A^{\prime}<A, R<R^{\prime}<\infty$, and $0<\varepsilon^{\prime}<\varepsilon$. Using Theorem 2, we obtain $P(z)=\mathcal{L}_{\alpha} F(t)$, where $F(t) \in B\left(R^{\prime \prime}, A^{\prime \prime}, \varepsilon^{\prime \prime}\right)$ for every $0<A^{\prime \prime}<A, R<R^{\prime \prime}<\infty$, and $0<\varepsilon^{\prime \prime}<\varepsilon$, and $G(t)=F(t, \alpha)$; see Theorem 2. It follows that

$$
\mathbf{B}(R, A, \varepsilon) \supset \mathcal{L}_{\alpha}^{-1} \mathbf{W}(R, A, \varepsilon) .
$$

8.3. Necessary and sufficient conditions for the Stokes phenomenon. Up to this point, we were assuming that $\varepsilon \in\left[0, \frac{\pi}{2}\right]$. Formulas (34), (35), and (36) allow us to consider the cases where $\varepsilon \in\left[\frac{\pi}{2}, \infty\right)$. All previous results, shown by Theorems $2-9$, can easily be extended to the case where $\varepsilon \in\left[\frac{\pi}{2}, \infty\right)$. Again, we demonstrate this for simplicity for the case of $R=0$.

Let $F(t) \in B(0, A, \varepsilon), \varepsilon \in\left[0, \frac{\pi}{2}\right]$. Let $\mathcal{P}(\varepsilon)$ be given by (216). Theorem $4 \mathrm{~A}$ and our analysis in Theorems $5 \mathrm{~A}$ and $6 \mathrm{~A}$ show that for $z \in \mathcal{P}(\varepsilon)$ the remainder $\mathrm{P}_{n}(z, \alpha)$, given by (99) with $d \sigma(\xi)=\frac{F(\xi) d \xi}{2 \pi i}$, can be represented in the form

$$
\mathrm{P}_{n}(z, \alpha) \equiv \frac{\Gamma(\alpha+n+1)}{z^{n+1}} \cdot \frac{1}{2 \pi i} \int_{\gamma(A, \varepsilon)} \frac{\widetilde{K}(-\xi z, \alpha+n) F(\xi) d \xi}{\xi^{n+1}}, \quad n \in \mathbb{Z}^{+},
$$


where $\widetilde{K}(-\xi z, \alpha+n)$ is given by (105), and

$$
-\frac{3 \pi}{2}<\arg (-\xi z)<\frac{3 \pi}{2}, \quad \xi \in \gamma(A, \varepsilon) .
$$

However, for $z \in \mathcal{P}(0)$ we have

$$
\mathrm{P}_{n}(z, \alpha) \equiv \frac{\Gamma(\alpha+n+1)}{z^{n+1}} \cdot \frac{1}{2 \pi i} \int_{\gamma(A, \varepsilon)} \frac{K(-\xi z, \alpha+n) F(\xi) d \xi}{\xi^{n+1}}, \quad n \in \mathbb{Z}^{+},
$$

where

$$
-\pi<\arg (-\xi z)<\pi, \quad \xi \in \gamma(A, \varepsilon) .
$$

The estimates for the basic kernel $K(-\xi z, \alpha+n)$ in the regions (242) and (243) imply the corresponding estimates for $\mathrm{P}_{n}(z, \alpha)$, given by (235) and (234), in the sectors $\mathcal{P}(\varepsilon)$ and $\mathcal{P}(0)$, respectively. It should be noted that in the sectors $\mathcal{P}^{+}(\varepsilon)$ and $\mathcal{P}^{-}(\varepsilon)$, given by (219) and (220), respectively, these estimates deteriorate, and for $\varepsilon=\frac{\pi}{2}$ and $\arg z=\frac{\pi}{2}$ these estimates lose meaning. It follows that the angles of the "deterioration" sectors $\mathcal{P}^{+}(\varepsilon)$ and $\mathcal{P}^{-}(\varepsilon)$ do not exceed $\frac{\pi}{2}$. This property is preserved if the function $F(t)$ admits analytic continuation to a larger sectorial region. The next theorem gives an exact result.

Theorem 10A. Assume that all conditions of Theorem 4A are satisfied. Suppose $F(t) \in$ $B(0, A, \varepsilon), \varepsilon \in\left[0, \frac{\pi}{2}\right]$, admits analytic continuation to the region $D\left(A, \frac{\pi}{2}+\theta\right), \theta \in\left[0, \frac{\pi}{2}\right)$, as an element of $B\left(0, A, \frac{\pi}{2}+\theta\right)$. Then $P_{n}(z, \alpha)$ admits analytic continuation from $\mathcal{P}(\varepsilon)$ to the region $\mathcal{P}(\pi+\theta)$, and for $z \in \mathcal{P}(\pi+\theta)$ we have

$$
\mathrm{P}_{n}(z, \alpha) \equiv \frac{\Gamma(\alpha+n+1)}{z^{n+1}} \cdot \frac{1}{2 \pi i} \int_{\partial \mathfrak{D}\left(A, \frac{\pi}{2}+\theta\right)} \frac{\widetilde{K}(-\xi z, \alpha+n) F(\xi) d \xi}{\xi^{n+1}}, \quad n \in \mathbb{Z}^{+},
$$

where $\partial \mathfrak{D}\left(A, \frac{\pi}{2}+\theta\right)$ is the boundary of $\mathfrak{D}\left(A, \frac{\pi}{2}+\theta\right)$, and

$$
-\frac{3 \pi}{2}<\arg (-\xi z)<\frac{3 \pi}{2}, \quad \xi \in \partial \mathfrak{D}\left(A, \frac{\pi}{2}+\theta\right) .
$$

For $z \in \mathcal{P}(\pi)$ we have

$$
\mathrm{P}_{n}(z, \alpha) \equiv \frac{\Gamma(\alpha+n+1)}{z^{n+1}} \cdot \frac{1}{2 \pi i} \int_{\partial \mathfrak{D}\left(A, \frac{\pi}{2}+\theta\right)} \frac{K(-\xi z, \alpha+n) F(\xi) d \xi}{\xi^{n+1}}, \quad n \in \mathbb{Z}^{+},
$$

and

$$
-\pi<\arg (-\xi z)<\pi, \quad \partial \mathfrak{D}\left(A, \frac{\pi}{2}+\theta\right) .
$$

Proof. Using Cauchy's theorem, we expand the contour $\gamma(A, \varepsilon)$ up to $\partial \mathfrak{D}\left(A, \frac{\pi}{2}+\theta\right)$, moving simultaneously $z$ from $\mathcal{P}(\varepsilon)$ to $\mathcal{P}(\pi+\theta)$ so that relations (242) and (243) continue to be true on $\partial \mathfrak{D}\left(A, \frac{\pi}{2}+\theta\right)$ as (244) and (245), respectively.

This allows us to extend easily all results of Theorems $4 \mathrm{~A}-9 \mathrm{~A}$ to the case where $\varepsilon \in\left[\frac{\pi}{2}, \infty\right)$. Moreover, the above analysis shows that the spaces $W\left(R, b, a, \theta_{1}, \theta_{2}\right)$ of analytic functions satisfying (13) and (14) make sense only if $\theta_{1}=\frac{n \pi}{2}$ and $\theta_{2}=\frac{n \pi}{2}+\theta$, where $n \in \mathbb{N}, \theta \in\left(0, \frac{\pi}{2}\right)$, and $a=b \cos \theta$. This gives statement 1 .

The above discussion allows us also to settle statement 8 .

Consider the following example. The function $F(t)=e^{-t^{2}}$ belongs to every space $B(0, A, \varepsilon)$ with $0 \leq \varepsilon<\frac{\pi}{4}$, and to every space $B\left(2 A, A, \frac{\pi}{4}\right)$ for an arbitrarily large value of $A, 0<A<\infty$. Theorem 4A, Theorem $6 \mathrm{~A}$, and Theorems 7-9 show that the function

$$
P(z)=\int_{0}^{\infty} e^{-z t} e^{-t^{2}} d t
$$


belongs to the space $W(0, A, \varepsilon)$ for every $0<A<\infty$ and $0<\varepsilon<\frac{\pi}{4}$, and to the space $W\left(2 A, A, \frac{\pi}{4}\right)$ for every $0<A<\infty$. Therefore, the function $F(t)$ generates a number of exponentially small terms $E_{A}^{ \pm}(z), z \in \mathcal{P}^{ \pm}(\varepsilon) \backslash \mathcal{P}$, with exponential factors $e^{i A z}$, $0<A<\infty$, given by (232) and (233), and every such term leads to an improvement of the estimate in the "deteriorated" regions. Generally, it is natural to derive this improvement with a maximal exponent. In the example (246), the condition max $A<\infty$ of the definition of Subsection 2.3 is violated.

Remark 10. In general, the function (246) drops out, in certain aspects, from our theory, and should be regarded as an element of the theory of expansions in which condition (2) in Nevanlinna's theorem is replaced by the condition 7

$$
\left|P(z)-\sum_{k=0}^{n-1} \frac{p_{k}}{z^{k+1}}\right| \leq \frac{M(n !)^{\frac{1}{\kappa}}}{A^{n}|z|^{n+1}}, \quad n=0,1, \ldots .
$$

These expansions with $0<\kappa<\infty$ were also studied by Nevanlinna, see [21], who found a characterization of the set $W_{\kappa}(R, A, \varepsilon)$ of $P(z)$ satisfying (247) in terms of the corresponding set $B_{\kappa}(R, A, \varepsilon)$ of functions $F(t)$ such that

$$
P(z)=\int_{0}^{\infty} e^{-(z t)^{2}} F(t) d t .
$$

Thus, for our case where $\kappa=2$, it is more natural to view $e^{-t^{2}}$ as an element of the space $B_{\kappa}(R, A, \varepsilon)$. Other functions, such as $F(t)=\frac{1}{\Gamma(z+1)}$ and $F(t)=e^{-e^{z}}$, which belong to $B(0, A, \pi)$ and $B\left(0, A, \frac{\pi}{2}\right)$, respectively, for every $A>0$, require an appropriate generalization of the Laplace transform. We note that the corresponding expansions are beyond the scope of Gevrey expansions of order $\kappa$.

\section{$\S 9$. Conclusion}

9.1. Error bounds. In 1965, F. Olver published the paper [39] in which he found rigorous bounds for the errors of asymptotic approximations for solutions of second-order differential equations having an irregular singularity of rank one.

Olver's paper is remarkable for two reasons.

- (i) First, Olver discovered that the principal sector in the complex plane in which the Poincaré asymptotic expansion holds can be split into a number of subsectors, and for each of these he found quite a precise estimate for the error, which gets worse as one approaches the boundary of the sector. In particular, from Olver's estimates it follows that the Poincaré asymptotic expansions for solutions of a differential equation are, in fact (if we use a now established terminology), Gevrey expansions with errors satisfying estimates similar to those shown in Subsection 2.2 for the Stirling expansion.

- (ii) Second, applying the method of successive approximations, in which the error under the integral sign should be replaced by the absolute value of the error, Olver derived surprisingly good error bounds.

To prove his results, Olver used his modification of the method of reduction of a differential equation to an equivalent singular integral equation. Olver's investigations were continued by Olver and Stenger [40, and by Stenger [41, 42, who studied this problem for the most general matrix equations with an irregular singularity of arbitrary rank. However, these papers were basically focused on the extension of Olver's method rather than on Olver's discovery in (ii), and their results are not so sharp as in [39].

${ }^{7}$ In the literature, the expansions satisfying (247) are sometimes called Gevrey expansions of order $\kappa$. 
Our approach, described in the current paper, occurred when we were looking for the possibility of finding an improvement and extension of Olver's estimates. We show now how Olver's success, as described above in (ii), can be explained. Let us return to the integral representation (138) for the basic kernel

$$
K\left(w e^{-i \pi}, s\right)=1-i(s+1) \int_{0}^{\infty} \frac{e^{i \lambda w}}{(1+i \lambda)^{s+2}} d \lambda .
$$

We have

$$
\left|K\left(w e^{-i \pi}, s\right)\right| \leq 1+(s+1) \int_{0}^{\infty} \frac{1}{\left(1+\lambda^{2}\right)^{\frac{s}{2}+1}} d \lambda,
$$

and the right-hand side of the last inequality can be rewritten as

$$
1+\sqrt{\pi} \frac{\Gamma\left(\frac{s}{2}+\frac{3}{2}\right)}{\Gamma\left(\frac{s}{2}+1\right)} .
$$

From Lemma 8 it follows that the right-hand sides of (248) and (249) have the same asymptotic behavior as $s \rightarrow \infty$, equal to $\sqrt{\frac{\pi s}{2}}$, and that (250) provides $\mu(s)$, given by (157), with a good approximation 8

The value (250) appeared first in [39] in error bounds for the confluent hypergeometric function, and then in [26] in error bounds for expansions generated by Bessel functions. In $\S 7$ we demonstrated the universality of Olver's numbers for a wider class of expansions.

Continuing Dingle's method of Borel summation of late terms for formal solutions of a differential equation, starting with the least term, Berry 3 proposed a method of "smoothing a Victorian discontinuity", which could explain and extend Olver's above results.

We started with a completely different idea of using the dual complex plane and a dual monodromy to obtain the error bounds.

9.2. Stokes' monodromic relations and Gauss' monodromic relations. We return to our Example 2 in Subsection 4.3, in which we considered the following representation for the Airy function:

$$
\operatorname{Ai}(z)=\frac{1}{2 \sqrt{\pi}} z^{-\frac{1}{4}} e^{-\zeta} P(\zeta),
$$

where $\zeta=\frac{2}{3} z^{\frac{3}{2}}$. It is not difficult to check that $P(\zeta)$ satisfies the following monodromic relation in the $z$-plane:

$$
P\left(\zeta e^{2 \pi i}\right)=P(\zeta)+i e^{2 \zeta} P\left(\zeta e^{\pi i}\right) .
$$

We have derived also, see (98), the representation

$$
P(\zeta)=\zeta \int_{0}^{+\infty} e^{-t \zeta}\left({ }_{2} F_{1}\left(\frac{1}{6}, \frac{5}{6}, 1 ;-\frac{t}{2}\right)\right) d t .
$$

The Euler linear transformation formula, see [30, yields the relation

$$
\begin{aligned}
& \left({ }_{2} F_{1}\left(\frac{1}{6}, \frac{5}{6}, 1 ; 1-\left(\frac{t}{2}+1\right) e^{\mp 2 \pi i}\right)\right) \\
& \quad=\left({ }_{2} F_{1}\left(\frac{1}{6}, \frac{5}{6}, 1 ;-\frac{t}{2}\right) \pm i\left({ }_{2} F_{1}\right)\left(\frac{1}{6}, \frac{5}{6}, 1 ; \frac{t}{2}+1\right)\right) ;
\end{aligned}
$$

see [51].

Given $a>0$ and a complex constant $T$, we introduce the relation

$$
P\left(\zeta e^{2 \pi i}\right)=P(\zeta)+T e^{a \zeta} P\left(\zeta e^{\pi i}\right) .
$$

\footnotetext{
${ }^{8} \mathrm{~A}$ better approximation can be obtained by using the expansion (155) of Lemma 5.
} 
Let us assume that

(i) $P(\zeta)$ is analytic at every point of the $\zeta$-plane except for $\zeta=0$ and $\zeta=\infty$;

(ii) given $P(\zeta)$, there exists a positive decreasing function $M(r)$ such that for all $r, 0<r<\infty$, if $\zeta$ satisfies $|\arg \zeta| \leq \pi$ and $|\zeta| \geq r$, then

$$
|P(\zeta)| \leq M(r) \text {. }
$$

Definition 1. Given $P(\zeta)$ with properties (i) and (ii), we call a relation of the form (253) a Stokes monodromic relation for $P(\zeta)$. The set of all functions $P(\zeta)$ that satisfy (i) and (ii) and also (253) forms a linear space denoted by $S_{a, T}$.

The following result holds.

Statement. Given $a>0$ and $T$, suppose that $P(\zeta) \in S_{a, T}$. Then

(i) there exists a function $F(t)$ supported on the positive ray of the $t$-plane such that for $|\arg \zeta|<\frac{\pi}{2}$ we have

$$
P(\zeta)=\zeta \int_{0}^{\infty} e^{-\zeta t} F(t) d t
$$

(ii) the function $F(t)$ can be continued analytically from the positive ray to the entire complex $t$-plane punctured at the two points $t=0$ and $t=-a$, with exponential growth at $\infty$ of minimal type in any sectorial region;

(iii) there exists a branch of $F(t)$ which is analytic in the t-plane cut along $(-\infty,-a]$;

(iv) we have the monodromic relation

$$
F^{*}(t)=F(t) \pm T F(-t-a) .
$$

Here $F^{*}(t)=F\left(t^{*}\right), t^{*}=-a+(t+a) e^{\mp 2 \pi i}$.

(v) The following relation is valid in a neighborhood of $t=-a$ :

$$
F(t)=-\frac{T F(0)}{2 \pi i} \log (t+a)+A+o(1), \quad t \rightarrow-a,
$$

where $A$ is a complex constant.

Definition 2. Given $a>0$ and $T$, and given $F(t)$ with properties (ii) (iii), and (iv) of the Statement, we call a relation of the form (255) a Gauss monodromic relation for $F(t)$. The set of all functions $F(t)$ that satisfy (ii) and (iii) of the Statement and also (255) forms a linear space denoted by $G_{a, T}$.

We can establish, in a more general setting, without any reference to differential equations, a one-to-one correspondence between monodromic relations of the two types (253) and (255). The properties of elements $F \in G_{a, T}$, which can be considered as generalized hypergeometric functions, allow us to associate with $P(\zeta)$, given by (254), its asymptotic expansion

$$
\sum_{k=0}^{\infty} p_{k} / \zeta^{k}, \quad p_{k}=F^{(k)}(0)
$$

and to derive sharp expressions for the error bounds using the technique shown above; see $[60]$.

Example 1. Consider a differential equation of the form

$$
\frac{d^{2} u}{d \zeta^{2}}=\left(\frac{a^{2}}{4}+\frac{1}{\zeta^{2}} A(\zeta)\right) u,
$$

where $a>0, A\left(\zeta^{-1}\right)$ is an even entire function so that

$$
A(\zeta)=a_{0}+\frac{a_{1}}{\zeta^{2}}+\cdots,
$$


$a_{0}, a_{1}, \ldots$ are complex numbers, and the series is convergent for any complex $\zeta$. This equation can be regarded as a perturbation of the standard Bessel equation, which reduces to the particular case of (258) with $a=2$ and $A(\zeta)=\frac{1}{4}-\nu^{2}$.

Representing the solution of (258) decaying on the positive ray in the form

$$
u(\zeta)=e^{-\frac{a}{2} \zeta} P(\zeta),
$$

it can be shown that $P(\zeta) \in S_{a, T}$, where $T=T\left(a_{0}, a_{1}, \ldots\right)$ is a complex constant which is usually referred to as the connection coefficient.

For the particular Bessel case this constant is known, $T=2 i \cos \nu \pi$, and if $\nu=\frac{1}{2}$, then $T=0$. The explicit expression for the connection coefficient $T\left(a_{0}, a_{1}, \ldots\right)$ in terms of the coefficients $\left\{p_{k}\right\}_{k=0}^{\infty}$, given by (257), follows from a more general results of [48] or 49]:

$$
T\left(a_{0}, a_{1}, \ldots\right)=\frac{2 \pi}{i} \lim _{t \rightarrow 1-0} \sum_{m=0}^{\infty} \frac{a^{m} t^{m+1}}{(m+1) !} \sum_{s=0}^{\left[\frac{m}{2}\right]}(-1)^{m-2 s} p_{m-2 s} a_{s}
$$

where $\left[\frac{m}{2}\right]$ is the integral part of $\frac{m}{2}$.

We aim to extend these results and to apply the extension to solutions of a wider class of differential equations. Combining these extensions with explicit formulas for connection coefficients that were shown in 48, and 49, and developing a technique initiated in [50] and [51, we are going to propose an alternative method of derivation of error bounds.

9.3. Duality, operating functions, and the Gamma function. The duality theorem allows us to show that the space $\mathbf{W}(R, A, \varepsilon)$ is closed under multiplication. Using the topological structure of this space, we expect that if $P(z) \in \mathbf{W}(R, A, \varepsilon)$, then $\exp P(z) \in \mathbf{W}(R, A, \varepsilon)$. It is clear that $P(z)$, given by (9), belongs to $\mathbf{W}(0,2 \pi, \pi)$. Thus, we can expect that $\exp P(z) \in \mathbf{W}(0,2 \pi, \pi)$.

This Stirling series for $\exp P(z)$, which arises as a factor of the asymptotic expansion for $\Gamma(z),-\pi<\arg z<\pi, z \rightarrow \infty$ :

$$
\Gamma(z) \sim \sqrt{2 \pi} z^{z-\frac{1}{2}} e^{-z} \sum_{k=n}^{\infty} \frac{a_{n}}{z^{n}}
$$

only became an object of investigation in the last half of the 20th century 9 Explicit expressions for the coefficients $a_{n}$ were presented in [47] and in [46]. The latter result is especially impressive:

$$
a_{n}=(2 n+1) ! ! b_{2 n+1},
$$

where $x$ !! is the double factorial, and the $b$-coefficients are defined by the conditions $b_{0}=b_{1}=1$ and the recurrence relations

$$
b_{n}=\frac{1}{n+1}\left(b_{n-1}-\sum_{k=2}^{n-1} k b_{k} b_{n+1-k}\right), \quad n=2, \ldots .
$$

A deep investigation of the error bounds for this expansion was done by W. G. C. Boyd [43. Boyd also studied the Stokes phenomenon for $\Gamma(z)$ upon crossing the positive and negative parts of the imaginary line. Boyd's study was based on the ideas and discoveries of Berry and Howls; see 44 and [45. A consequence of Boyd's study was the observation that many properties of Stirling's series for $\Gamma(z)$ and $\log \Gamma(z)$ are very similar. Boyd's explanation of the Stokes phenomenon for $\Gamma(z)$ is as clear as a similar

\footnotetext{
${ }^{9}$ We quote Olver [26] (1974), p. 88, "No general expression is available for the coefficients. The first twenty-one have been given by Wrench in 1968, together with approximate value of the next ten."
} 
explanation for $\log \Gamma(z)$ given by Berry. However, it should be noted that Boyd used the reflection formula to study consequences of the Stokes phenomenon upon crossing the imaginary line. Thus, he missed the opportunity of finding the exponentially small additive corrections, given by (221) and (222), which appear upon crossing the Stokes lines.

The function $P(z)=\frac{1}{\sqrt{2 \pi}} \Gamma(z) z^{-z+\frac{1}{2}} e^{-z}-1$ can be represented as the Laplace transform of a function $F(t)$ analytic in the entire $t$-plane except for the set $\{2 \pi i k\}_{k \in \mathbb{Z}, k \neq 0}$. For the case of $k=0$, the values of the Taylor coefficients of $F(t)$ at $z=0$ follow from the expressions for $a_{n}$. Given $k, k \neq 0$, the function $F(t)$ in the disk of radius $2 \pi$ centered at $2 \pi i k$ can be represented in the form $F(t)=\log (t-2 \pi i k) \sum_{l=0}^{\infty} a_{k, l}(t-2 \pi i k)^{l}$, and the series is absolutely convergent. Therefore, our general method can be applied again to prove that $F(t) \in B\left(0,2 \pi, \frac{\pi}{2}\right)$ and to evaluate the exponentially small functions $P^{ \pm}(z)$ in terms of the coefficients $a_{k, l}$. The problem of the evaluation of the coefficients $a_{k, l}$ is more difficult - the work of Boyd may be of assistance here.

9.4. Stirling expansion for $\log \Gamma(z)$ (historical notes). No series has had a longer history or has undergone a more thorough exploration than the Stirling series. In modern times the properties of this series are generally accepted as established and fundamental results, as can be seen in contemporary 10 monographs and handbooks on asymptotic expansions, such as [54, 25, 26, 33, 55, 56, 6], etc. However, certain principal questions related to the exactness of the error bounds for the Stirling expansion have remained unanswered. Answers to these questions suggest similar results for more general expansions in the complex plane.

Although interest in such error bounds for the Stirling expansion has continued unabated up to the present time, the most hectic activity in this subject occurred in the nineteenth century. Nielsen's monograph 36 referred to about four hundred publications of about 150 mathematicians, including the original monograph by Stirling, with a description of his two methods.

In 1886, Stieltjes published the paper [52], in which he undertook a systematic study of the error for a number of divergent expansions, including the Stirling expansion. He called these, and similar expansions, semiconvergent expansions. Three years later in [53, he proved that for every $z,-\pi<\arg z<\pi, \theta=\arg z$, and for all $n \in \mathbb{N}$, the terminant, given by (12), satisfies the inequality

$$
|T(z, n)| \leq \sec ^{2 n}\left(\frac{\theta}{2}\right) .
$$

In particular, for $\theta= \pm \frac{\pi}{2}$ we have

$$
|T(z, n)| \leq 2^{n} .
$$

The analysis of the Stieltjes method by Lindelöf [35] showed that for $-\frac{\pi}{2}<\arg z<\frac{\pi}{2}$ we have an estimate which does not depend on $n$,

$$
|T(z, n)| \leq \frac{1}{|\sin 2 \theta|},
$$

and from (266), by using the maximum principle, it follows that in the smaller sector $-\frac{\pi}{2}<\arg z<\frac{\pi}{2}$ we have $T(z, n) \leq 1$. A comparison of (265) with (266) emphasizes the need for a better estimate on the cuts $\arg z=\frac{\pi}{2}$ and $\arg z=-\frac{\pi}{2}$, which, in fact, are the Stokes lines for the Stirling expansion. The best known estimate for the error on these

${ }^{10}$ Published in the second part of the twentieth century. 
cuts has remained that of [35], page 101: for all $z, \arg z= \pm \frac{\pi}{2}$ we have

$$
|T(z, n)| \leq \sqrt{\frac{e(n+1)}{2}}, \quad n \in \mathbb{N} .
$$

The available literature shows that the 20th century investigations have added almost nothing to this result. Compare it, for example, with the estimates in [57, 58, 55, 59, 33. Lindelöf's result remained unsurpassed for over 100 years. However, this result, which clearly indicates the relationship of the Stokes phenomenon for the Stirling expansion with the Sokhotsky-Plemelj formula has been forgotten.

In our paper we prove that for all $z$ belonging to the closed right half-plane we have

$$
|T(z, n)| \leq 1+\sqrt{\frac{\pi}{4}\left(n+\frac{1}{2}\right)}, \quad n \in \mathbb{N},
$$

and (268) is better than (267) (which is obvious for larger $n$, since $\frac{\pi}{4}<\frac{e}{2}$ ). We prove also that the coefficient $\frac{\pi}{4}$ in (268) cannot be replaced by a smaller number.

\section{§10. ACKNOWLEDGMENTS}

The author is grateful to Valery Pokrovsky for many insightful discussions during the last decade. The author is also thankful to his colleagues David Gillam, David Lucy, Nick Garnham, Sergey Suslov and John Beynon for their valuable suggestions. When the paper was being prepared, the author was invited to discuss the material with Victor Katsnelson, Vladimir Matsaev, and Dmitry Novikov at the Weizmann Institute of Science. The author is very grateful for their useful and insightful criticism and comments. Finally, the author expresses his gratitude to Anton Baranov who checked the proofs of the most principal results in this paper.

\section{REFERENCES}

[1] R. E. Meyer, A simple explanation of the Stokes phenomenon, SIAM Rev. 31 (1989), no. 3, 435-445. MR.1012299 (90f:34097)

[2] M. V. Berry, Stokes' phenomenon; smoothing a Victorian discontinuity, Inst. Hautes Études Sci. Publ. Math. No. 68 (1988), 211-221 (1989). MR1001456 (90j:58019)

[3] _ Uniform asymptotic smoothing of Stokes's discontinuities, Proc. Roy. Soc. London Ser. A 422 (1989), 7-21. MR0990851 (90h:34084)

[4] _ Infinitely many Stokes smoothings in the gamma function, Proc. Roy. Soc. London Ser. A 434 (1991), 465-472. MR.1121933 (92h:33002)

[5] _ . Asymptotics, superasymptotics, hyperasymptotics ..., Asymptotics Beyond All Orders (La Jolla, CA, 1991), NATO Adv. Sci. Inst. Ser. B Phys., vol. 284, Plenum, New York, 1991, pp. 1-14. MR 1210328

[6] R. B. Paris and D. Kaminski, Asymptotics and Mellin-Barnes integrals, Encyclopedia Math. Appl., vol. 85, Cambridge Univ. Press, Cambridge, 2001. MR.1854469 (2002h:33001)

[7] J.-P. Ramis, Séries divergentes et théories asymptotiques, Bull. Soc. Math. France 121 (1993), Panoramas et Syntheses, Suppl., 74 pp. MR.1272100 (95h:34074)

[8] Stokes phenomenon: historical background, The Stokes Phenomenon and Hilbert's 16th Problem (Groningen, 1995) (B. L. J. Braaksma, G. K. Immink, M. Van der Put, eds.), World Sci. Publ., River Edge, NJ, 1996, pp. 1-5. MR1443685

[9] R. B. Paris and A. D. Wood, Stokes phenomenon demystified, Bull. Inst. Math. Appl. 31 (1995), 21-28. MR 1323501

[10] G. G. Stokes, On the numerical calculation of a class of definite integrals and infinite series, Trans. Camb. Philos. Soc. 9 (1850), 166-187; On the discontinuity of arbitrary constants which appear in divergent developments, Trans. Camb. Philos. Soc. 10 (1857), 106-128; Memoir and scientific correspondence, selected and arranged by J. Larmor, Vol. II, Cambridge Univ. Press, London-New York, 1907, pp. 159-160.

[11] V. L. Pokrovskiĭ and I. M. Khalatnikov, On the problem of above-barrier reflection of high-energy particles, Zh. Èksper. Teoret. Fiz. 40 (1961), no. 6, 1713-1719; English transl., Soviet Phys. JETP 13 (1961), 1207-1210. 
[12] J. P. Boyd, The devil's invention: Asymptotic, superasymptotic and hyperasymptotic series, Acta Appl. Math. 56 (1999), no. 1, 1-98. MR.1698036 (2000f:34102)

[13] V. P. Gurariı̆, Group methods in commutative harmonic analysis, Itogi Nauki i Tekhniki Sovrem. Probl. Mat. Fundam. Naprav., vol. 25, VINITI, Moscow, 1988, pp. 4-303; English transl., Encyclopaedia Math. Sci., vol. 25, Springer, Berlin, 1998, pp. 1-325. MR0982753 (90f:43001) MR 1622490

[14] G. N. Watson, A theory of asymptotic series, Philos. Trans. Roy. Soc. London Ser. A 211 (1911), 279-313.

[15] F. Nevanlinna, Zur Theorie der asymptotischen Potenzreihen, Ann. Acad. Sci. Fenn. Ser. A 12 (1916), no. 1.

[16] T. Carleman, Les fonctions quasi-analytiques, Gauthier-Villars, Paris, 1926.

[17] G. H. Hardy, Divergent series, Clarendon Press, Oxford, 1949. MR0030620 (11:25a)

[18] R. B. Dingle, Asymptotic expansions and converging factors. I. General theory and basic converging factors; II. Error, Dawson, Fresnel, exponential, sine and cosine, and similar integrals; III. Gamma, psi and polygamma functions, and Fermi-Dirac and Bose-Einstein integrals, Proc. Roy. Soc. London Ser. A 244 (1958), 456-475, 476-483, 484-490. MR0103373 (21:2145) MR0103374 (21:2146) MR0103375 (21:2147)

[19] - Asymptotic expansions and converging factors. IV. Confluent hypergeometric, parabolic cylinder, modified Bessel, and ordinary Bessel functions; V. Lommel, Struve, modified Struve, Anger and Weber functions, and integrals of ordinary and modified Bessel functions; VI. Application to physical prediction, Proc. Roy. Soc. London Ser. A 249 (1959), 270-283, 284-292, 293-295. MR0103376(21:2148a) MR0103377(21:2148b) MR0103378(21:2148c)

[20] _ Asymptotic expansions: their derivation and interpretation, Academic Press, London-New York, 1973. MR0499926 (58:17673)

[21] F. Nevanlinna, Zur Theorie der asymptotischen Potenzreihen, Ann. Acad. Sci. Fenn. Ser. A 12 (1918), no. 3, 1-81.

[22] L. Bieberbach, Algemeine Theorie der Funktionen komplexer Argumente. Kapital 4, Jahrb. Forts. Math. 46 (1916-1918), no. 1.

[23] A. D. Sokal, An improvement of Watson's theorem on Borel summability, J. Math. Phys. 21 (1980), no. 2, 261-263. MR0558468 (81h:4011)

[24] Jorge Rezende, A note on Borel summability, J. Math. Phys. 34 (1993), no. 9, 4330-4339. MR.1233275 (94g:40003)

[25] H. Jeffreys, Asymptotic approximation, Clarendon Press, Oxford, 1962. MR0147821 (26:5334)

[26] F. W. J. Olver, Asymptotics and special functions, Academic Press, New York-London, 1974. MR0435697 (55:8655)

[27] D. W. H. Gillam and V. P. Gurariū, On functions uniquely determined by their asymptotic expansion, Funktsional. Anal. i Prilozhen. 40 (2006), no. 4, 33-48; English transl., Funct. Anal. Appl. 40 (2006), no. 4, 273-284. MR2307701(2008a:30070)

[28] Herbert Buchholz, Die konfluente hypergeometrische Funktion mit besonderer Berücksichtigung ihrer Anwendungen, Ergeb. Angew. Math., Bd. 2, Springer-Verlag, Berlin, 1953; English transl., The confluent hypergeometric function with special emphasis on its applications, Springer Tracts in Natural Philos., vol. 15, Springer-Verlag New York Inc., New York, 1969. MR0054783 (14:978e) MR 0240343 (39:1692)

[29] D. W. H. Gillam and V. P. Gurariı̌, Parametric representations in the theory of Laplace-Mellin transforms (in preparation).

[30] G. E. Andrews, R. Askey, and R. Roy, Special functions, Encyclopedia Math. Appl., vol. 71, Cambridge Univ. Press, Cambridge, 1999. MR 1688958 (2000g:33001)

[31] M. Abramowitz and I. Stegun (eds.), Handbook of mathematical functions with formulas, graphs and mathematical tables, Dover Publ., Inc., New York, 1992. MR1225604 (94b:00012)

[32] E. T. Copson, An introduction to the theory of a complex variable, Oxford Univ. Press, 1962.

[33] Y. L. Luke, The special functions and their approximations. Vol. 1, Math. Sci. Engrg., vol. 53, Academic Press, New York-London, 1969. MR0241700 (39:3039)

[34] Ch. Hermite, Sur une application du théorème de M. Mittag-Leffler, dans la théorie des fonctions, J. Crelle 92 (1882), 145-155.

[35] E. Lindelöf, Le calcul des résidus et ses applications à la théorie des fonctions, Gauthier-Villars, Paris, 1905. MR:1189798 (93f:30001)

[36] N. Nielsen, Handbuch der Theorie der Gammafunktion, Teubner, Leipzig, 1906. MR0185152 $(32: 2622)$

[37] A. B. Olde Daalhuis, Hyperasymptotic expansions of confluent hypergeometric functions, IMA J. Appl. Math. 49 (1992), 203-216. MR1198790(94e:33002) 
[38] H. Poincaré, Sur les intégrales irrégulières. Des équations linéaires, Acta Math. 8 (1886), no. 1, 295-344. MR 1554701

[39] F. W. J. Olver, On the asymptotic solution of second-order differential equations having an irregular singularity of rank one, with an application to Whittaker functions, J. Soc. Indust. Appl. Math. Ser. B Numer. Anal. 2 (1965), no. 2, 225-243. MR0185350 (32:2818)

[40] F. W. J. Olver and F. Stenger, Error bounds for asymptotic solutions of second-order differential equations having an irregular singularity of arbitrary rank, J. Soc. Indust. Appl. Math. Ser. B Numer. Anal. 2 (1965), no. 2, 244-249. MR0185351 (32:2819)

[41] F. Stenger, Error bounds for asymptotic solutions of differential equations. I. The distinct eigenvalue case, J. Res. Nat. Bur. Standards Sect. B 70B (1966), 167-186. MR0219823 (36:2901)

[42] __ Error bounds for asymptotic solutions of differential equations. II. The general case, J. Res. Nat. Bur. Standards Sect. B 70B (1966), 187-210. MR0219824 (36:2902)

[43] W. G. C. Boyd, Gamma function asymptotics by an extension of the method of steepest descents, Proc. Roy. Soc. London Ser. A 447 (1994), 609-630. MR1316625 (96c:33002)

[44] M. V. Berry and C. J. Howls, Hyperasymptotics, Proc. Roy. Soc. London Ser. A 430 (1990), 653-668. MR 1070081 (91i:34064)

[45] _ Hyperasymptotics for integrals with saddles, Proc. Roy. Soc. London Ser. A 434 (1991), 657-675. MR $1126872(92 \mathrm{j}: 41041)$

[46] J. M. Borwein and R. M. Corless, Emerging tools for experimental mathematics, Amer. Math. Monthly 106 (1999), 889-909. MR.1732501 (2000m:68186)

[47] L. Comtet, Advanced combinatorics. The art of finite and infinite expansions, D. Reidel Publ. Co., Dordrecht, 1974. MR0460128 (57:124)

[48] V. P. Gurariı̆ and V. I. Matsaev, Stokes multipliers for systems of first-order linear ordinary differential equations, Dokl. Akad. Nauk SSSR 280 (1985), no. 2, 272-276; English transl., Soviet Math. Dokl. 31 (1985), no. 1, 52-56. MR0775049 (86k:34019)

[49] _ The generalized Borel transform and Stokes multipliers, Teoret. Mat. Fiz. 100 (1994), no. 2, 173-182; English transl., Theoret. and Math. Phys. 100 (1994), no. 2, 928-936 (1995). MR1311191 (95k:34006)

[50] V. P. Gurariŭ, J. Steiner, V. Katsnelson, and V. Matsaev, How to use the Fourier transform in asymptotic analysis, Twentieth Century Harmonic Analysis - a Celebration (Il Ciocco, 2000), NATO Sci. Ser. II Math. Phys. Chem., vol. 33, Kluwer Acad. Publ., Dordrecht, 2001, pp. 387-401. MR $1858792(2003 \mathrm{e}: 34165)$

[51] V. P. Gurariĭ and D. Lucy, The Stokes structure and connection coefficients for the Airy equation, Mat. Fiz. Anal. Geom. 10 (2003), no. 3, 385-411. (English) MR2012270 (2004i:34236)

[52] T. J. Stieltjes, Recherches sur quelques séries semi-convergentes, Ann. Sci. Ecole. Norm. Sup. (3) 3 (1886), 201-258. MR.1508781

[53] Sur le développement de $\log \Gamma(a)$, J. Math. (4) 5 (1889), 425-444.

[54] N. G. de Bruijn, Asymptotic methods in analysis, Bibliotheca Math., vol. 4, North-Holland Publ. Co., Amsterdam; P. Noordhoff Ltd., Groningen, 1958. MR0099564 (20:6003)

[55] È. Ya. Riekstyn'sh, Estimates for remainders in asymptotic expansions, Zinatne, Riga, 1986. (Russian) MR0875418 (88c:41052)

[56] Z. X. Wang and D. R. Guo, Special functions, World Sci. Publ. Co., Inc., Teaneck, NJ, 1989. MR 1034956 (91a:33001)

[57] J. G. van der Corput, Asymptotics. II. Elementary methods, Nederl. Akad. Wetensch. Proc. Ser. A 58 (1955), 139-150. MR 0073739 (17:478b)

[58] R. Spira, Calculation of the gamma function by Stirling's formula, Math. Comp. 25 (1971), 317322. MR0295539 (45:4605)

[59] A. Erdélyi, W. Magnus, F. Oberhettinger, and F. Tricomi, Higher transcendental functions, McGraw-Hill Book Co., Inc., New York, 1953. MR0058756 (15:419i)

[60] V. P. Gurariı and D. W. H. Gillam, Stokes and Gauss monodromic relations and the duality theorem, Preprint, Monash Univ., 2010 (to appear).

Mathematics, Faculty of Engineering and Industrial Sciences, Swinburne University of Technology, PO Box 218, Hawthorn 3122, and School of Mathematical Sciences, Monash University, Clayton 3800, VIC, Australia

E-mail address: vgurarii@swin.edu.au; vladimir.gurarii@sci.monash.edu.au

Received 4/OCT/2009

Originally published in English 